\title{
TARGETED SEQUENTIAL DESIGN FOR TARGETED LEARNING INFERENCE OF THE OPTIMAL TREATMENT RULE AND ITS MEAN REWARD / SUPPLEMENTAL ARTICLE
}

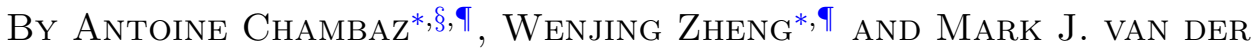

$$
\mathrm{LAAN}^{\dagger, \boldsymbol{\top}}
$$

UPL, Université Paris Nanterre ${ }^{\S}$ and University of California, Berkeley

The proofs of the results stated in the main article are gathered in Section A. They hinge on technical lemmas presented and proved in Section B. Two additional lemmas about the pathwise differentiability of two mappings central to our analysis are recalled in Section C for completeness. A figure and a table summarizing the results of the simulation study form Section D. Finally, a notation index is given in Section E, to help the reader travel through the main article.

\section{APPENDIX A: PROOFS}

The notation $a \lesssim b$ means that expression $a$ is smaller than expression $b$ up to a universal multiplicative constant.

To alleviate notation, we introduce the indexing parameter $\zeta \in \cup_{n \geq 1} B_{n} \times$ $\mathcal{G}_{1}$ which stands for a couple $(\beta, g)$. For every $\zeta \equiv(\beta, g) \in \cup_{n \geq 1} B_{n} \times \mathcal{G}_{1}$, $\rho \in \mathcal{R}$ and $\epsilon \in \mathcal{E}$, we set

$$
Q_{Y, \zeta, \rho}(\epsilon) \equiv \operatorname{expit}\left(\operatorname{logit}\left(Q_{Y, \beta}\right)+\epsilon H_{\rho}(g)\right)
$$

and characterize $Q_{Y, \zeta, \rho}(\epsilon) \circ \rho$ given by

$$
Q_{Y, \zeta, \rho}(\epsilon) \circ \rho(W)=Q_{Y, \zeta, \rho}(\epsilon)(\rho(W), W) .
$$

With $\zeta_{n} \equiv\left(\beta_{n}, g_{n}\right)$ and $\zeta_{0} \equiv\left(\beta_{0}, g_{0}\right)$, we set

$$
\begin{aligned}
Q_{Y, \zeta_{n}, r_{n}}^{*} & \equiv Q_{Y, \zeta_{n}, r_{n}}\left(\epsilon_{n}\right), \\
Q_{Y, \zeta_{0}, r_{n}}^{*} & \equiv Q_{Y, \zeta_{0}, r_{n}}\left(\epsilon_{0}\left(r_{n}\right)\right)
\end{aligned}
$$

* Supported by the French Agence Nationale de la Recherche (ANR), under grant ANR13-BS01- 0005 (project SPADRO).

${ }^{\dagger}$ This research has been conducted as part of the project Labex MME-DII (ANR11LBX-0023-01).

${ }^{\ddagger}$ Supported by NIH Grant Number 2R01 A1074345-07. 
where $\epsilon_{0}\left(r_{n}\right)$ is defined in (4.3) with $\rho \equiv r_{n}$. With both $\zeta=\zeta_{n}$ and $\zeta=\zeta_{0}$, we also introduce $Q_{Y, \zeta, r_{n}}^{*} \circ r_{n}$ and $d_{Y, \zeta, r_{n}}^{*}$ given by

$$
\begin{aligned}
Q_{Y, \zeta, r_{n}}^{*} \circ r_{n}(W) & \equiv Q_{Y, \zeta, r_{n}}^{*}\left(r_{n}(W), W\right), \\
d_{Y, \zeta, r_{n}}^{*}(O, Z) & \equiv \frac{\mathbf{1}\left\{A=r_{n}(W)\right\}}{Z}\left(Y-Q_{Y, \zeta, r_{n}}^{*}(A, W)\right) .
\end{aligned}
$$

In particular, $d_{Y, \zeta_{n}, r_{n}}^{*}=d_{Y, n}^{*}$ previously defined in (4.13). Finally, we denote $Q_{\zeta, r_{n}}^{*}$ any $Q \in \mathcal{Q}$ such that the marginal distribution of $W$ under $Q$ is the empirical measure and $Q_{Y}=Q_{Y, \zeta, r_{n}}^{*}$.

Lemmas 3.1, 3.2 and 3.3 are proven in Section A.1. Proposition 4.1 and Theorem 4.2 in Section A.2 and Propositions 5.2, 5.3 and 5.4 in Section A.3. Technical lemmas are presented and proven in Section B.

\section{A.1. Proofs of Lemmas 3.1, 3.2 and 3.3.}

Proof of Lemma 3.1. The key to the proof is the following identity: for each $g \in \mathcal{G}$, we have

$$
E_{Q_{0}, g}\left(Q_{Y, 0}(A, W)\right)=E_{Q_{0}}\left(Q_{Y, 0}(0, W)\right)+E_{Q_{0}}\left(q_{Y, 0}(W) g(1 \mid W)\right) .
$$

This is a straightforward consequence of the decomposition $Q_{Y, 0}(A, W)=$ $Q_{Y, 0}(0, W)+A q_{Y, 0}(W)$. Moreover, (A.5) also holds when $g$ takes its value in $[0,1]$, hence for all treatment rules (TRs) as well.

Set $n \geq 1$. Applying (A.5) with $g=r_{n}$ and $g=r_{0}$ yields

$$
\begin{aligned}
& E_{Q_{0}, r_{n}}\left(Q_{Y, 0}(A, W)\right)=E_{Q_{0}}\left(Q_{Y, 0}(0, W)\right)+E_{Q_{0}}\left(q_{Y, 0}(W) r_{n}(W)\right), \\
& E_{Q_{0}, r_{0}}\left(Q_{Y, 0}(A, W)\right)=E_{Q_{0}}\left(Q_{Y, 0}(0, W)\right)+E_{Q_{0}}\left(q_{Y, 0}(W) r_{0}(W)\right) .
\end{aligned}
$$

Because $E_{Q_{0}, r_{0}}\left(Q_{Y, 0}(A, W)\right)=E_{Q_{0}}\left(Q_{Y, 0}\left(r_{0}(W), W\right)\right)=\psi_{0}$, subtracting (A.6) and (A.7) entails

$$
\begin{aligned}
\psi_{0}-E_{Q_{0}, r_{n}}\left(Q_{Y, 0}(A, W)\right) & =E_{Q_{0}}\left(q_{Y, 0}(W) \times\left(r_{0}(W)-r_{n}(W)\right)\right) \\
& \leq\left\|r_{n}-r_{0}\right\|_{1} .
\end{aligned}
$$

By definition of $r_{0}$, the above LHS expression is non-negative, hence it coincides with $\Delta\left(r_{n}, r_{0}\right)$. This completes the proof of (3.6).

We now apply (A.5) with $g=g_{0}$ to get

$$
E_{Q_{0}, g_{0}}\left(Q_{Y, 0}(A, W)\right)=E_{Q_{0}}\left(Q_{Y, 0}(0, W)\right)+E_{Q_{0}}\left(q_{Y, 0}(W) g_{0}(1 \mid W)\right) .
$$

Subtracting (A.9) and (A.7) yields the new equality

$$
0 \leq \psi_{0}-E_{Q_{0}, g_{0}}\left(Q_{Y, 0}(A, W)\right)=E_{Q_{0}}\left(q_{Y, 0}(W) \times\left(r_{0}(W)-g_{0}(1 \mid W)\right)\right) .
$$


Based on (3.2), a case-by-case study depending on the sign of $q_{Y, 0}(W)$ finally reveals that

$$
0 \leq \psi_{0}-E_{Q_{0}, g_{0}}\left(Q_{Y, 0}(A, W)\right) \leq t_{\infty} E_{Q_{0}}\left(\left|q_{Y, 0}(W)\right|\right)+\xi_{\infty} \leq t_{\infty}+\xi_{\infty}
$$

To obtain (3.7), we simply note that

$$
\begin{aligned}
0 \leq & \psi_{0}-E_{Q_{0}, g_{n}}\left(Q_{Y, 0}(A, W)\right) \\
= & \psi_{0}-E_{Q_{0}, g_{0}}\left(Q_{Y, 0}(A, W)\right) \\
& \quad+E_{Q_{0}, g_{0}}\left(Q_{Y, 0}(A, W)\right)-E_{Q_{0}, g_{n}}\left(Q_{Y, 0}(A, W)\right) \\
\leq & t_{\infty}+\xi_{\infty}+\Delta\left(g_{n}, g_{0}\right)
\end{aligned}
$$

by (A.10) and (3.5).

Proof of Lemma 3.2. Set $n \geq 1, p \geq 1$ and $\eta>0$. There exists $\alpha>0$ such that $P_{Q_{0}}\left(0<\left|q_{Y, 0}(W)\right|<\alpha\right) \leq \eta^{p} / 2$.

Note that $\left|\left(r_{n}-r_{0}\right)(W)\right| \in\{0,1\}$. Moreover, $\left|\left(r_{n}-r_{0}\right)(W)\right|=1$ implies $q_{Y, \beta_{n}} q_{Y, 0}(W) \leq 0$. This justifies the first inequality below. The others easily follow from the fact that $\left|q_{Y, 0}(W)\right| \leq 1$ and a case-by-case study depending on whether $0<\left|q_{Y, 0}(W)\right|<\alpha$ or not:

$$
\begin{aligned}
\left|q_{Y, 0}(W)\right| \times\left|\left(r_{n}-r_{0}\right)(W)\right|^{p} \leq & \left|q_{Y, 0}(W)\right| \times \mathbf{1}\left\{q_{Y, \beta_{n}} q_{Y, 0}(W) \leq 0\right\} \\
\leq & \mathbf{1}\left\{0<\left|q_{Y, 0}(W)\right|<\alpha\right\}+\mathbf{1}\left\{\left|q_{Y, 0}(W)\right| \geq \alpha\right\} \\
& \times\left|q_{Y, 0}(W)\right| \times \mathbf{1}\left\{\left|\left(q_{Y, \beta_{n}}-q_{Y, 0}\right)(W)\right| \geq \alpha\right\} \\
\leq & \mathbf{1}\left\{0<\left|q_{Y, 0}(W)\right|<\alpha\right\}+\mathbf{1}\left\{\left|q_{Y, 0}(W)\right| \geq \alpha\right\} \\
& \times\left|q_{Y, 0}(W)\right| \times \alpha^{-1}\left|\left(q_{Y, \beta_{n}}-q_{Y, 0}\right)(W)\right| \\
\leq & \mathbf{1}\left\{0<\left|q_{Y, 0}(W)\right|<\alpha\right\} \\
& +\alpha^{-1}\left|q_{Y, 0}(W)\right|^{1 / 2} \times\left|\left(q_{Y, \beta_{n}}-q_{Y, 0}\right)(W)\right| .
\end{aligned}
$$

Taking the expectation under $Q_{W, 0} d \mu_{W}$ on both sides yields

$$
\begin{aligned}
& \left\|r_{n}-r_{0}\right\|_{p}^{p} \leq P_{Q_{0}}\left(0<\left|q_{Y, 0}(W)\right|<\alpha\right) \\
& \quad+\alpha^{-1} \int\left|q_{Y, 0}\right|^{1 / 2} \times\left|\left(q_{Y, \beta_{n}}-q_{Y, 0}\right)\right| Q_{W, 0} d \mu_{W}
\end{aligned}
$$

hence, by choice of $\alpha$ and the Cauchy-Schwartz inequality,

$$
\left\|r_{n}-r_{0}\right\|_{p}^{p} \leq \eta^{p} / 2+\alpha^{-1}\left\|q_{Y, \beta_{n}}-q_{Y, 0}\right\|_{2} .
$$

Therefore, $\left\|r_{n}-r_{0}\right\|_{p} \geq \eta$ implies $\left\|q_{Y, \beta_{n}}-q_{Y, 0}\right\|_{2} \geq \alpha \eta^{p} / 2$. Consequently, $\left\|q_{Y, \beta_{n}}-q_{Y, 0}\right\|_{2}=o_{P}(1)$ does yield $\left\|r_{n}-r_{0}\right\|_{p}=o_{P}(1)$. This completes the proof. 
Proof of Lemma 3.3. Set $n \geq 1, p \geq 1, \bar{p}=p /(p-1)(\bar{p}=\infty$ if $p=1)$ and $p^{\prime}=\min (p, 2)$.

By (A.5) with $g=g_{n}$ and $g=g_{0}$, we obtain

$$
\Delta\left(g_{n}, g_{0}\right)=\left|E_{Q_{0}}\left[q_{Y, 0}(W) \times\left(g_{n}(1 \mid W)-g_{0}(1 \mid W)\right)\right]\right| .
$$

Applying successively the triangle inequality and Hölder's inequality yields

$$
\begin{aligned}
\Delta\left(g_{n}, g_{0}\right) & \leq E_{Q_{0}}\left(\left|q_{Y, 0}(W)\right| \times\left|g_{n}(1 \mid W)-g_{0}(1 \mid W)\right|\right) \\
& \leq\left\|g_{n}-g_{0}\right\|_{p},
\end{aligned}
$$

which is the result first stated in the lemma.

Suppose now that $n$ is large enough so that $G_{n}=G_{\infty}$. Since $G_{\infty}$ is $c_{\infty}$-Lipschitz, it holds that

$$
\begin{aligned}
\left|q_{Y, 0}(W)\right| \times \mid g_{n}(1 \mid W) & -\left.g_{0}(1 \mid W)\right|^{p} \\
& =\left|q_{Y, 0}(W)\right| \times\left|G_{\infty}\left(q_{Y, \beta_{n}}(W)\right)-G_{\infty}\left(q_{Y, 0}(W)\right)\right|^{p} \\
& \lesssim\left|q_{Y, 0}(W)\right| \times\left|q_{Y, \beta_{n}}(W)-q_{Y, 0}(W)\right|^{p} \\
& \leq\left|q_{Y, 0}(W)\right| \times\left|q_{Y, \beta_{n}}(W)-q_{Y, 0}(W)\right|^{p^{\prime}}
\end{aligned}
$$

where the last inequality is due to the fact that $\left|q_{Y, \beta_{n}}-q_{Y, 0}\right| \leq 1$. Taking the expectation under $Q_{W, 0} d \mu_{W}$ gives the bound $\left\|g_{n}-g_{0}\right\|_{p} \lesssim\left\|q_{Y, \beta_{n}}-q_{Y, 0}\right\|_{p^{\prime}}^{p^{\prime} / p} \lesssim$ $\left\|q_{Y, \beta_{n}}-q_{Y, 0}\right\|_{2}^{p^{\prime} / p}$. This completes the proof.

A.2. Proofs of Proposition 4.1 and Theorem 4.2. Let us prove Proposition 4.1.

Proof of Proposition 4.1. The convergence $\left\|q_{Y, \beta_{n}}-q_{Y, \beta_{0}}\right\|=o_{P}(1)$

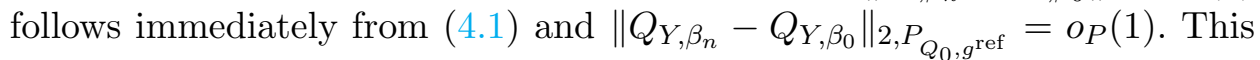
convergence is a consequence of Lemma B.1 with $\Theta \equiv \mathcal{Q}_{1}, \Theta_{n} \equiv \mathcal{Q}_{1, n}, d$ the

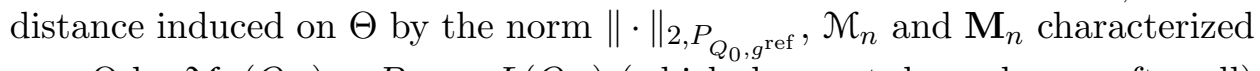
over $\Theta$ by $\mathcal{M}_{n}\left(Q_{Y}\right) \equiv P_{Q_{0}, g^{\text {ref }}} L\left(Q_{Y}\right)$ (which does not depend on $n$ after all) and $\mathbf{M}_{n}\left(Q_{Y}\right) \equiv P_{n} g^{\mathrm{ref}} L\left(Q_{Y}\right) / Z=n^{-1} \sum_{i=1}^{n} g^{\mathrm{ref}}\left(A_{i} \mid W_{i}\right) L\left(Q_{Y}\right)\left(O_{i}\right) / Z_{i}$. Assumption A2 implies that (a) and (b) from Lemma B.1 are met (take $\tau_{n}=Q_{Y, \beta_{0}}$ and $\tau_{n}^{*}=Q_{Y, \beta_{n, 0}}$. It remains to prove that (c) also holds or, in other terms, that $\left\|\mathbf{M}_{n}-\mathcal{M}_{n}\right\|_{\mathcal{Q}_{1, n}}=o_{P}(1)$.

For any $Q_{Y} \in \Theta$, characterize $\ell\left(Q_{Y}\right)$ by setting

$$
\ell\left(Q_{Y}\right)(O, Z) \equiv g^{\mathrm{ref}}(A \mid W) L\left(Q_{Y}\right)(O) / Z .
$$

Then we can rewrite $\left\|\mathbf{M}_{n}-\mathcal{M}_{n}\right\|_{\mathcal{Q}_{1, n}}$ as follows: 


$$
\begin{aligned}
\left\|\mathbf{M}_{n}-\mathcal{M}_{n}\right\|_{\mathcal{Q}_{1, n}}=\| P_{n} \ell & -P_{Q_{0}, g^{\mathrm{ref}}} L \|_{\mathcal{Q}_{1, n}} \\
& =\left\|\left(P_{n}-P_{Q_{0}, \mathbf{g}_{n}}\right) \ell\right\|_{\mathcal{Q}_{1, n}}=\left\|P_{n}-P_{Q_{0}, \mathbf{g}_{n}}\right\|_{\ell\left(\mathcal{Q}_{1, n}\right)} .
\end{aligned}
$$

The separability of $\ell\left(\mathcal{Q}_{1, n}\right)$ follows from that of $L\left(\mathcal{Q}_{1, n}\right)$. Let $F_{n}$ be the envelope function for $L\left(\mathcal{Q}_{1, n}\right)$ from $\mathbf{A} 4$. By construction of $g_{n}, Z$ is bounded away from 0 , so there exists a constant $c>0$ such that $c F_{n}$ is an envelope function for $\ell\left(\mathcal{Q}_{1, n}\right)$. Moreover, $J_{c F_{n}}\left(1, \ell\left(\mathcal{Q}_{1, n}\right)\right)=O\left(J_{F_{n}}\left(1, L\left(\mathcal{Q}_{1, n}\right)\right)=o(\sqrt{n})\right.$ by A4. Therefore, Lemma B.3 applies and yields $\left\|P_{n}-P_{Q_{0}, \mathbf{g}_{n}}\right\|_{\ell\left(\mathcal{Q}_{1, n}\right)}=o_{P}(1)$ by Markov's inequality. Thus, we can apply Lemma B.1. It yields that $\left\|Q_{Y, \beta_{n}}-Q_{Y, \beta_{0}}\right\|_{2, P_{Q_{0}, g^{\text {ref }}}}=o_{P}(1)$, which is the desired result.

Assume now that $\mathbf{A} \mathbf{1}$ and $\mathbf{A} 5$ also hold and set arbitrarily $t>0$. Because $\left|r_{n}-r_{0}\right| \in\{0,1\}$, we can upper-bound $\left\|r_{n}-r_{0}\right\|_{2, P_{Q_{0}, g^{\text {ref }}}}^{2}$ as follows:

$$
\begin{aligned}
\left\|r_{n}-r_{0}\right\|_{2, P_{Q_{0}, g^{\mathrm{ref}}}}^{2}= & P_{Q_{0}, g^{\mathrm{ref}}} \mathbf{1}\left\{\left|q_{Y, 0}\right|>t\right\} \times\left|r_{n}-r_{0}\right| \\
& \quad+P_{Q_{0}, g^{\mathrm{ref}}} \mathbf{1}\left\{\left|q_{Y, 0}\right| \leq t\right\} \times\left|r_{n}-r_{0}\right| \\
\leq & t^{-1} P_{Q_{0}, g^{\mathrm{ref}}}\left|q_{Y, 0}\right| \times\left|r_{n}-r_{0}\right|+P_{Q_{0}, g^{\mathrm{ref}}}\left(0<\left|q_{Y, 0}\right| \leq t\right) \\
\lesssim & t^{-1}\left\|r_{n}-r_{0}\right\|_{2}^{2}+t^{\gamma_{2}} .
\end{aligned}
$$

Optimizing in $t$ yields

$$
\left\|r_{n}-r_{0}\right\|_{2, P_{Q_{0}, g^{\mathrm{ref}}}} \lesssim\left\|r_{n}-r_{0}\right\|_{2}^{\gamma_{2} / 2\left(1+\gamma_{2}\right)}=o_{P}(1) .
$$

We obtain that

$$
\left\|g_{n}-g_{0}\right\|_{2, P_{Q_{0}, g^{\mathrm{ref}}}} \lesssim\left\|g_{n}-g_{0}\right\|_{2}^{\gamma_{2} / 2\left(1+\gamma_{2}\right)}=o_{P}(1)
$$

along the same lines as above. This completes the proof.

We now turn to the first part of Theorem 4.2:

Proposition A.1 (consistency of $\psi_{n}^{*}$ ). Suppose that A2, A3 and A4 are met. Then it holds that $\psi_{n}^{*}-\psi_{r_{n}, 0}=o_{P}(1)$.

Proof of Proposition A.1. This is a three-part proof.

Step one: studying $\epsilon_{n}$. Let us show that $\epsilon_{n}-\epsilon_{0}\left(r_{n}\right)=o_{P}(1)$. We apply Lemma B.2 with $\Theta \equiv \mathcal{E}, d$ the Euclidean distance, $\boldsymbol{Z}_{n}$ and $\mathbf{Z}_{n}$ characterized over $\mathcal{E}$ by $\mathcal{Z}_{n}(\epsilon)=P_{Q_{0}, g_{0}} D_{Y, r_{n}}\left(Q_{Y, \zeta_{0}, r_{n}}(\epsilon), g_{0}\right)$, and

$$
\mathbf{Z}_{n}(\epsilon)=P_{n} D_{Y, r_{n}}\left(Q_{Y, \zeta_{n}, r_{n}}(\epsilon), g_{n}\right) g_{n} / Z,
$$

see (A.1), (4.2) and (2.15) for the definitions of $Q_{Y, \zeta_{0}, r_{n}}(\epsilon)$ and $Q_{Y, \zeta_{n}, r_{n}}(\epsilon)$. 
From the differentiability of $\epsilon \mapsto L^{\mathrm{kl}}\left(Q_{Y, \zeta_{0}, r_{n}}(\epsilon)\right)$, validity of the differentiation under the integral sign, and definition of $\epsilon_{0}\left(r_{n}\right)$ (4.3) in A3, we deduce that $z_{n}\left(\epsilon_{0}\left(r_{n}\right)\right)=0$. By definition of $\epsilon_{n}(2.16), \mathbf{Z}_{n}\left(\epsilon_{n}\right)=0$ too. Moreover, (d) from Lemma B.2 is met. Indeed, by differentiability of $\epsilon \mapsto D_{Y, r_{n}}\left(Q_{Y, \zeta_{0}, r_{n}}(\epsilon), g_{0}\right)$ and validity of the differentiation under the integral sign, $Z_{n}: \mathcal{E} \rightarrow \mathbb{R}$ is differentiable on $\mathcal{E}$ with a derivative given by

$$
z_{n}^{\prime}(\varepsilon)=-P_{Q_{0}, g_{0}} \frac{Q_{Y, \zeta_{0}, r_{n}}(\epsilon) \circ r_{n} \times\left(1-Q_{Y, \zeta_{0}, r_{n}}(\epsilon) \circ r_{n}\right)}{g_{0} \circ r_{n}}
$$

where $g_{0} \circ r_{n}$ is characterized by $g_{0} \circ r_{n}(W)=g_{0}\left(r_{n}(W) \mid W\right)$. By construction, $Q_{Y, \zeta, r}(\epsilon)$ and $g_{0}$ are bounded away from 0 and 1 uniformly in $\zeta \in \cup_{n \geq 1} B_{n} \times$ $\mathcal{G}_{1}, \rho \in \mathcal{R}$ and $\epsilon \in \mathcal{E}$. Therefore, there exists a universal constant $c$ such that $\left|Z_{n}^{\prime}(\varepsilon)\right| \geq c>0$ for all $\epsilon \in \mathcal{E}$. Consequently, by the mean value theorem, for all $\varepsilon \in \mathcal{E},\left|\mathcal{Z}_{n}(\epsilon)\right| \geq c\left|\epsilon-\epsilon_{0}\left(r_{n}\right)\right|$. This entails condition (d).

Applying Lemma B.2 finally requires verifying that $(e)$ is met, i.e., proving that $\left\|\mathbf{Z}_{n}-\mathcal{z}_{n}\right\|_{\mathcal{E}}=o_{P}(1)$. Introduce $\mathcal{F}_{n} \equiv\left\{f_{\rho, \epsilon}: \rho \in r\left(\mathcal{Q}_{1, n}\right), \epsilon \in \mathcal{E}\right\}$ with

$$
f_{\rho, \epsilon}(O, Z) \equiv \frac{\mathbf{1}\{A=\rho(W)\}}{Z}\left(Y-Q_{Y, \zeta_{0}, \rho}(\epsilon)(A, W)\right)
$$

for each $(\rho, \epsilon) \in r\left(\mathcal{Q}_{1, n}\right) \times \mathcal{E}$. We start with the following derivation:

$$
\begin{aligned}
& \left\|\mathbf{Z}_{n}(\epsilon)-Z_{n}(\epsilon)\right\|_{\mathcal{E}} \\
& =\sup _{\epsilon \in \mathcal{E}} \mid P_{n}\left(f_{r_{n}, \epsilon}+\frac{\mathbf{1}\left\{A=r_{n}(W)\right\}}{Z}\left(Q_{Y, \zeta_{0}, r_{n}}(\epsilon)-Q_{Y, \zeta_{n}, r_{n}}(\epsilon)\right)\right) \\
& -P_{Q_{0}, \mathbf{g}_{n}} f_{r_{n}, \epsilon} \mid \\
& \leq\left\|P_{n}-P_{Q_{0}, \mathbf{g}_{n}}\right\|_{\mathcal{F}_{n}} \\
& +\sup _{\epsilon \in \mathcal{E}}\left|P_{n} \frac{\mathbf{1}\left\{A=r_{n}(W)\right\}}{Z}\left(Q_{Y, \zeta_{0}, r_{n}}(\epsilon)-Q_{Y, \zeta_{n}, r_{n}}(\epsilon)\right)\right| .
\end{aligned}
$$

- Consider the first RHS term in (A.12). Set $\left(\rho_{1}, \epsilon_{1}\right),\left(\rho_{2}, \epsilon_{2}\right) \in r\left(\mathcal{Q}_{1, n}\right) \times$ $\mathcal{E}$. Since $Z$ is bounded away from 0 and $\left|Y-Q_{Y, \zeta_{0}, \rho_{2}}\left(\epsilon_{2}\right)(A, W)\right| \leq 1$, it holds that

$$
\begin{aligned}
\left.\left|\left(f_{\rho_{1}, \epsilon_{1}}-f_{\rho_{2}, \epsilon_{2}}\right)(O, Z)\right| \leq \frac{\mathbf{1}\{A}{=} \rho_{1}(W)\right\} \\
Z \\
\times\left|\left(Q_{Y, \zeta_{0}, \rho_{1}}\left(\epsilon_{1}\right)-Q_{Y, \zeta_{0}, \rho_{2}}\left(\epsilon_{2}\right)\right)(A, W)\right| \\
+\mid \mathbf{1}\left\{A=\rho_{1}(W)-\mathbf{1}\left\{A=\rho_{2}(W)\right\} \mid\right. \\
\times \frac{\left|Y-Q_{Y, \zeta_{0}, \rho_{2}}\left(\epsilon_{2}\right)(A, W)\right|}{Z}
\end{aligned}
$$




$$
\begin{aligned}
\lesssim & \left|\left(Q_{Y, \zeta_{0}, \rho_{1}}\left(\epsilon_{1}\right)-Q_{Y, \zeta_{0}, \rho_{1}}\left(\epsilon_{2}\right)\right)(A, W)\right| \\
& +\left|\left(Q_{Y, \zeta_{0}, \rho_{1}}\left(\epsilon_{2}\right)-Q_{Y, \zeta_{0}, \rho_{2}}\left(\epsilon_{2}\right)\right)(A, W)\right| \\
& +\left|\rho_{1}(W)-\rho_{2}(W)\right| .
\end{aligned}
$$

Because expit is 1-Lipschitz, $\mathcal{E}$ is bounded and $g_{0}$ is bounded away from 0 , this entails the bound

$$
\begin{aligned}
\left|\left(f_{\rho_{1}, \epsilon_{1}}-f_{\rho_{2}, \epsilon_{2}}\right)(O, Z)\right| \lesssim & \left|\epsilon_{1}-\epsilon_{2}\right| \\
& +\left|\epsilon_{2}\right| \times\left|\left(H_{\rho_{1}}\left(g_{0}\right)-H_{\rho_{2}}\left(g_{0}\right)\right)(O)\right| \\
& \quad+\left|\rho_{1}(W)-\rho_{2}(W)\right| \\
& \lesssim\left|\epsilon_{1}-\epsilon_{2}\right|+\left|\rho_{1}(W)-\rho_{2}(W)\right| .
\end{aligned}
$$

This upper-bound notably implies that $\mathcal{F}_{n}$ is separable because $r\left(\mathcal{Q}_{1, n}\right)$ and $\mathcal{E}$ (seen as a class of constant functions) are separable. By $\mathbf{A} 4$, $J_{1}\left(1, r\left(\mathcal{Q}_{1, n}\right)\right)=o(\sqrt{n})$. Since $\mathcal{E}$ is bounded, there exists a bounded envelope function $F$ for $\mathcal{E}$ seen as a class of (constant) functions and $J_{F}(1, \mathcal{E})$ is finite. Assume without loss of generality that $F$ is also an envelope function for $\mathcal{F}_{n}$. By (A.13) and the trivial inequalities $(a+b)^{2} \leq 2\left(a^{2}+b^{2}\right)$ and $\sqrt{a+b} \leq \sqrt{a}+\sqrt{b}$ (valid for all $a, b \geq$ 0 ), $J_{F}\left(1, \mathcal{F}_{n}\right)=o(\sqrt{n})$ (we will use repeatedly this argument in the rest of the article, without mentioning its details). Therefore, we can apply Lemma B.3 and conclude, with Markov's inequality, that $\| P_{n}-$ $P_{Q_{0}, \mathbf{g}_{n}} \|_{\mathcal{F}_{n}}=o_{P}(1)$.

- Consider next the second term in the RHS of (A.12). It is upperbounded by

$$
\Delta_{n} \equiv \sup _{\epsilon \in \mathcal{E}} P_{n}\left|Q_{Y, \zeta_{0}, r_{n}}(\epsilon)-Q_{Y, \zeta_{n}, r_{n}}(\epsilon)\right| / Z
$$

Since expit is 1-Lipschitz, $\mathcal{Q}_{1, n}$ is bounded away from 0 and 1 , and logit is Lipschitz on any compact subset of $] 0,1\left[\right.$, it holds that $\Delta_{n}$ is smaller than

$$
\begin{aligned}
\sup _{\epsilon \in \mathcal{E}} P_{n} & \left|\operatorname{logit}\left(Q_{Y, \beta_{n}}\right)-\operatorname{logit}\left(Q_{Y, \beta_{0}}\right)+\epsilon\left(H_{r_{n}}\left(g_{n}\right)-H_{r_{n}}\left(g_{0}\right)\right)\right| / Z \\
\lesssim & P_{n}\left|Q_{Y, \beta_{n}}-Q_{Y, \beta_{0}}\right| / Z+P_{n}\left|1 / g_{n}-1 / g_{0}\right| / Z \\
= & P_{Q_{0}, \mathbf{g}_{n}}\left|Q_{Y, \beta_{n}}-Q_{Y, \beta_{0}}\right| / Z+P_{Q_{0}, \mathbf{g}_{n}}\left|1 / g_{n}-1 / g_{0}\right| / Z \\
& +\left(P_{n}-P_{Q_{0}, \mathbf{g}_{n}}\right)\left|Q_{Y, \beta_{n}}-Q_{Y, \beta_{0}}\right| / Z \\
& +\left(P_{n}-P_{Q_{0}, \mathbf{g}_{n}}\right)\left|1 / g_{n}-1 / g_{0}\right| / Z .
\end{aligned}
$$

Using the fact that $g^{\text {ref }}$ is bounded away from 0 and 1 and the CauchySchwarz inequality, we readily see that $P_{Q_{0}, \mathbf{g}_{n}}\left|Q_{Y, \beta_{n}}-Q_{Y, \beta_{0}}\right| / Z \lesssim$ 
$P_{Q_{0}, g^{\text {ref }}}\left|Q_{Y, \beta_{n}}-Q_{Y, \beta_{0}}\right| \leq\left\|Q_{Y, \beta_{n}}-Q_{Y, \beta_{0}}\right\|_{2, P_{Q_{0}, g^{\text {ref }}}}=o_{P}(1)$ by Proposition 4.1, whose assumptions are met here. We control $P_{Q_{0}, \mathbf{g}_{n}} \mid 1 / g_{n}-$ $1 / g_{0} \mid / Z$ similarly, using additionally that $\mathbf{g}_{n}$ and $g_{0}$ are uniformly bounded away from 0 and 1 and that, for $n$ large enough, $G_{n}=G_{\infty}$ is $c_{\infty}$-Lipschitz. Indeed, for $n$ large enough, $P_{Q_{0}, \mathbf{g}_{n}}\left|1 / g_{n}-1 / g_{0}\right| / Z \lesssim$ $P_{Q_{0}, g^{\text {ref }}}\left|g_{n}-g_{0}\right| \leq\left\|g_{n}-g_{0}\right\|_{2, P_{Q_{0}, g^{\text {ref }}}}$ and

$$
\begin{aligned}
\left\|g_{n}-g_{0}\right\|_{2, P_{Q_{0}, g^{\mathrm{ref}}}} & =\left\|G_{\infty}\left(q_{Y, \beta_{n}}\right)-G_{\infty}\left(q_{Y, \beta_{0}}\right)\right\|_{2, P_{Q_{0}, g^{\mathrm{ref}}}} \\
& \lesssim\left\|q_{Y, \beta_{n}}-q_{Y, \beta_{0}}\right\|_{2, P_{Q_{0}, \mathrm{ref}^{\mathrm{ref}}}} \\
& \lesssim\left\|Q_{Y, \beta_{n}}-Q_{Y, \beta_{0}}\right\|_{2, P_{Q_{0}, g^{\mathrm{ref}}}}=o_{P}(1),
\end{aligned}
$$

as recalled earlier. Thus, the sum of the two first terms in the RHS expression of (A.14) is $o_{P}(1)$.

We now turn to the third term of the RHS sum in (A.14). For any $Q_{Y} \in$ $\mathcal{Q}_{1}$, introduce $h_{1}\left(Q_{Y}\right)$ characterized by $h_{1}\left(Q_{Y}\right)(O, Z) \equiv \mid Q_{Y}(A, W)-$ $Q_{Y, \beta_{0}}(A, W) \mid / Z$. Obviously,

$$
\begin{aligned}
\left|\left(P_{n}-P_{Q_{0}, \mathbf{g}_{n}}\right)\right| Q_{Y, \beta_{n}}-Q_{Y, \beta_{0}}|/ Z| & \leq\left\|\left(P_{n}-P_{Q_{0}, \mathbf{g}_{n}}\right) h_{1}\right\|_{\mathcal{Q}_{1, n}} \\
& =\left\|P_{n}-P_{Q_{0}, \mathbf{g}_{n}}\right\|_{h_{1}\left(\mathcal{Q}_{1, n}\right)} .
\end{aligned}
$$

The separability of $\mathcal{Q}_{1, n}$ implies that of $h_{1}\left(\mathcal{Q}_{1, n}\right)$. Since $Z$ is bounded away from 0 , it holds that $h_{1}\left(\mathcal{Q}_{1}\right)$ is uniformly bounded by a constant $c>0$ which can serve as a constant envelope function, and

$$
\begin{aligned}
J_{c}\left(1, h_{1}\left(\mathcal{Q}_{1, n}\right)\right)=O\left(J _ { 1 } \left(1,\left\{\mid Q_{Y}-Q_{Y, \beta_{0}}\right.\right.\right. & \left.\left.\left.: Q_{Y} \in \mathcal{Q}_{1, n} \mid\right\}\right)\right) \\
& =O\left(J_{1}\left(1, \mathcal{Q}_{1, n}\right)\right)=o(\sqrt{n})
\end{aligned}
$$

by A4. Therefore, Lemma B.3 applies and Markov's inequality yields $\left\|P_{n}-P_{Q_{0}, \mathbf{g}_{n}}\right\|_{h_{1}\left(\mathcal{Q}_{1, n}\right)}=o_{P}(1)$. We control the last term similarly. Let $n$ be large enough so that $G_{n}=G_{\infty}$. For any $Q_{Y} \in \mathcal{Q}_{1}$, introduce $h_{2}\left(Q_{Y}\right)$ characterized by $h_{2}\left(Q_{Y}\right)(O, Z) \equiv \mid 1 / G_{\infty}\left(q_{Y}(A, W)\right)-$ $1 / G_{\infty}\left(q_{Y, \beta_{0}}(A, W)\right) \mid / Z$. We have

$$
\begin{aligned}
\left|\left(P_{n}-P_{Q_{0}, \mathbf{g}_{n}}\right)\right| 1 / g_{n}-1 / g_{0}|/ Z| & \leq\left\|\left(P_{n}-P_{Q_{0}, \mathbf{g}_{n}}\right) h_{2}\right\|_{\mathcal{Q}_{1, n}} \\
& =\left\|P_{n}-P_{Q_{0}, \mathbf{g}_{n}}\right\|_{h_{2}\left(\mathcal{Q}_{1, n}\right)} .
\end{aligned}
$$

The separability of $\mathcal{Q}_{1, n}$ implies that of $h_{2}\left(\mathcal{Q}_{1, n}\right)$. Because $Z$ is bounded away from 0 and because $G_{\infty}$ is $c_{\infty}$-Lipschitz and bounded away from 0 and 1 too, it holds that $h_{2}\left(\mathcal{Q}_{1}\right)$ is uniformly bounded by a constant $c^{\prime}>0$ which can serve as a constant envelope function, and 


$$
\begin{aligned}
& J_{c^{\prime}}\left(1, h_{2}\left(\mathcal{Q}_{1, n}\right)\right)=O\left(J_{1}\left(1,\left\{\left|q_{Y}-q_{Y, \beta_{0}}\right|: Q_{Y} \in \mathcal{Q}_{1, n}\right\}\right)\right) \\
= & O\left(J_{1}\left(1,\left\{\left|Q_{Y}-Q_{Y, \beta_{0}}\right|: Q_{Y} \in \mathcal{Q}_{1, n}\right\}\right)\right)=O\left(J_{1}\left(1, \mathcal{Q}_{1, n}\right)\right)=o(\sqrt{n}),
\end{aligned}
$$

as we have seen before. Thus, $\left\|P_{n}-P_{Q_{0}, \mathbf{g}_{n}}\right\|_{h_{2}\left(\mathcal{Q}_{1, n}\right)}=o_{P}(1)$, hence the sum of the tow last terms in the RHS expression of (A.14) is $o_{P}(1)$. We conclude that $\Delta_{n}=o_{P}(1)$.

Combining the results obtained on the first and second RHS terms in (A.12) yields the desired convergence $\left\|\mathbf{Z}_{n}-z_{n}\right\|_{\mathcal{E}}=o_{P}(1)$. We are now in a position to apply Lemma B.1, which implies the stated convergence $\epsilon_{n}-\epsilon_{0}\left(r_{n}\right)=$ $o_{P}(1)$.

Step two: studying $Q_{Y, \zeta_{n}, r_{n}}^{*}$. Let us now prove that

$$
\left\|Q_{Y, \zeta_{n}, r_{n}}^{*}-Q_{Y, \zeta_{0}, r_{n}}^{*}\right\|_{2, P_{Q_{0}, g} \text { ref }}=o_{P}(1) .
$$

For this, we equip $\mathcal{Q}_{1} \times \mathcal{G}_{1} \times \mathcal{E}-\mathcal{Q}_{1} \times \mathcal{G}_{1} \times \mathcal{E}$ with a seminorm $\|\cdot\|_{1}$ such that, for any two $\left(Q_{Y, 1}, g_{1}, \epsilon_{1}\right),\left(Q_{Y, 2}, g_{2}, \epsilon_{2}\right) \in \mathcal{Q}_{1} \times \mathcal{G}_{1} \times \mathcal{E}$,

$$
\begin{aligned}
\left\|\left(Q_{Y, 1}, g_{1}, \epsilon_{1}\right)-\left(Q_{Y, 2}, g_{2}, \epsilon_{2}\right)\right\|_{1} \equiv & \left\|Q_{Y, 1}-Q_{Y, 2}\right\|_{2, P_{Q_{0}, g^{\mathrm{ref}}}} \\
& +\left\|g_{1}-g_{2}\right\|_{2, P_{Q_{0}, g^{\mathrm{ref}}}}+\left|\epsilon_{1}-\epsilon_{2}\right| .
\end{aligned}
$$

Proposition 4.1 and the first step of this proof imply that

$$
\left\|\left(Q_{Y, \beta_{n}}, g_{n}, \epsilon_{n}\right)-\left(Q_{Y, \beta_{0}}, g_{0}, \epsilon_{0}\left(r_{n}\right)\right)\right\|_{1}=o_{P}(1) .
$$

We also equip the set $\mathcal{Q}_{Y}^{\mathcal{R}}-\mathcal{Q}_{Y}^{\mathcal{R}}$ with a seminorm $\|\cdot\|_{2}$ characterized as follows: for any two $\left(Q_{Y, \rho}\right)_{\rho \in \mathcal{R}},\left(Q_{Y, \rho}^{\prime}\right)_{\rho \in \mathcal{R}} \in \mathcal{Q}_{Y}^{\mathcal{R}}$,

$$
\left\|\left(Q_{Y, \rho}\right)_{\rho \in \mathcal{R}}-\left(Q_{Y, \rho}^{\prime}\right)_{\rho \in \mathcal{R}}\right\|_{2} \equiv \sup _{\rho \in \mathcal{R}}\left\|Q_{Y, \rho}-Q_{Y, \rho}^{\prime}\right\|_{2, P_{Q_{0}, g^{\mathrm{ref}}}} .
$$

Let $\boldsymbol{f}: \mathcal{Q}_{1} \times \mathcal{G}_{1} \times \mathcal{E} \rightarrow \mathcal{Q}_{Y}^{\mathcal{R}}$ be given by $\boldsymbol{f}\left(Q_{Y}, g, \epsilon\right)=\left(f_{\rho}\left(Q_{Y}, g, \epsilon\right)\right)_{\rho \in \mathcal{R}}$ where, for each $\rho \in \mathcal{R}$,

$$
f_{\rho}\left(Q_{Y}, g, \epsilon\right)(O) \equiv \operatorname{expit}\left(\operatorname{logit}\left(Q_{Y}(A, W)\right)+\epsilon H_{\rho}(g)(O)\right) .
$$

Set $\left(Q_{Y, 1}, g_{1}, \epsilon_{1}\right),\left(Q_{Y, 2}, g_{2}, \epsilon_{2}\right) \in \mathcal{Q}_{1} \times \mathcal{G}_{1} \times \mathcal{E}$ and $\rho \in \mathcal{R}$. Because (i) expit is 1-Lipschitz, (ii) $\mathcal{Q}_{1}$ is bounded away from 0 and 1, and logit is Lipschitz on any compact subset of $] 0,1\left[\right.$, (iii) $\mathcal{G}_{1}$ is uniformly bounded away from 0 and $1,(i v) \mathcal{E}$ is a bounded set, it holds that

$$
\begin{aligned}
& \left\|f_{\rho}\left(Q_{Y, 1}, g_{1}, \epsilon_{1}\right)-f_{\rho}\left(Q_{Y, 2}, g_{2}, \epsilon_{2}\right)\right\|_{2, P_{Q_{0}, g^{\mathrm{ref}}}} \\
& \quad \leq\left\|\operatorname{logit}\left(Q_{Y, 1}\right)-\operatorname{logit}\left(Q_{Y, 2}\right)\right\|_{2, P_{Q_{0}, g^{\mathrm{ref}}}}+\left\|\epsilon_{2}\left(1 / g_{1}-1 / g_{2}\right)\right\|_{2, P_{Q_{0}, g^{\mathrm{ref}}}}
\end{aligned}
$$




$$
\begin{aligned}
& +\left\|\left(\epsilon_{1}-\epsilon_{2}\right) / g_{1}\right\|_{2, P_{Q_{0}, g^{\mathrm{ref}}}} \\
& \lesssim\left\|Q_{Y, 1}-Q_{Y, 2}\right\|_{2, P_{Q_{0}, g^{\mathrm{ref}}}}+\left\|g_{1}-g_{2}\right\|_{2, P_{Q_{0}, g^{\mathrm{ref}}}}+\left|\epsilon_{1}-\epsilon_{2}\right| \\
(\mathrm{A} .17) & =\left\|\left(Q_{Y, 1}, g_{1}, \epsilon_{1}\right)-\left(Q_{Y, 2}, g_{2}, \epsilon_{2}\right)\right\|_{1} .
\end{aligned}
$$

Noting that the RHS expression does not depend on $\rho$ then taking the supremum in $\rho \in \mathcal{R}$ to the left yields

$$
\left\|\boldsymbol{f}\left(Q_{Y, 1}, g_{1}, \epsilon_{1}\right)-\boldsymbol{f}\left(Q_{Y, 2}, g_{2}, \epsilon_{2}\right)\right\|_{2} \lesssim\left\|\left(Q_{Y, 1}, g_{1}, \epsilon_{1}\right)-\left(Q_{Y, 2}, g_{2}, \epsilon_{2}\right)\right\|_{1} .
$$

Therefore, the convergence $\left\|\left(Q_{Y, \beta_{n}}, g_{n}, \epsilon_{n}\right)-\left(Q_{Y, \beta_{0}}, g_{0}, \epsilon_{0}\left(r_{n}\right)\right)\right\|_{1}=o_{P}(1)$ implies the convergence $\left\|\boldsymbol{f}\left(Q_{Y, \beta_{n}}, g_{n}, \epsilon_{n}\right)-\boldsymbol{f}\left(Q_{Y, \beta_{0}}, g_{0}, \epsilon_{0}\left(r_{n}\right)\right)\right\| \|_{2}=o_{P}(1)$. In particular,

$$
\begin{aligned}
& \left\|f_{r_{n}}\left(Q_{Y, \beta_{n}}, g_{n}, \epsilon_{n}\right)-f_{r_{n}}\left(Q_{Y, \beta_{0}}, g_{0}, \epsilon_{0}\left(r_{n}\right)\right)\right\|_{2, P_{Q_{0}, g^{\mathrm{ref}}}} \\
& =\left\|Q_{Y, \zeta_{n}, r_{n}}^{*}-Q_{Y, \zeta_{0}, r_{n}}^{*}\right\|_{2, P_{Q_{0}, g^{\mathrm{ref}}}}=o_{P}(1),
\end{aligned}
$$

as we claimed.

Step three: studying $\psi_{n}^{*}$. Let us first demonstrate that $E_{Q_{W, 0}}\left(Q_{Y, \zeta_{0}, r_{n}}^{*} \circ\right.$ $\left.r_{n}(W)\right)=\psi_{r_{n}, 0}$, then that $\psi_{n}^{*}-\psi_{r_{n}, 0}=o_{P}(1)$. We have already shown that $z_{n}\left(\epsilon_{0}\left(r_{n}\right)\right)=0$. Equivalently, by conditioning first on $(A, W)$ (second line) then on $W$ only (third line),

$$
\begin{aligned}
0 & =z_{n}\left(\epsilon_{0}\left(r_{n}\right)\right) \\
& =E_{Q_{0}, g_{0}}\left(\frac{\mathbf{1}\left\{A=r_{n}(W)\right\}}{g_{0}(A \mid W)}\left(Y-Q_{Y, \zeta_{0}, r_{n}}^{*}(A, W)\right)\right) \\
& =E_{Q_{0}, g_{0}}\left(\frac{\mathbf{1}\left\{A=r_{n}(W)\right\}}{g_{0}(A \mid W)}\left(Q_{Y, 0}(A, W)-Q_{Y, \zeta_{0}, r_{n}}^{*}(A, W)\right)\right) \\
& =E_{Q_{0}, g_{0}}\left(Q_{Y, 0}\left(r_{n}(W), W\right)-Q_{Y, \zeta_{0}, r_{n}}^{*}\left(r_{n}(W), W\right)\right) \\
(\mathrm{A} .18) & =\psi_{r_{n}, 0}-E_{Q_{W, 0}}\left(Q_{Y, \zeta_{0}, r_{n}}^{*} \circ r_{n}(W)\right)
\end{aligned}
$$

hence the claimed equality.

Let $\psi_{n}^{\sim} \equiv E_{Q_{W, 0}}\left(Q_{Y, \zeta_{n}, r_{n}}^{*} \circ r_{n}(W)\right)$. By (A.18), the fact that $g^{\text {ref }}$ is bounded away from 0 and 1 and the Cauchy-Schwarz inequality, it holds that

$$
\begin{aligned}
& \left|\psi_{n}^{\sim}-\psi_{r_{n}, 0}\right| \\
& =\left|E_{Q_{W, 0}, g^{\text {ref }}}\left(\frac{1\left\{A=r_{n}(W)\right\}}{g^{\mathrm{ref}}(A \mid W)}\left(Q_{Y, \zeta_{n}, r_{n}}^{*}-Q_{Y, \zeta_{0}, r_{n}}^{*}\right)(A, W)\right)\right| \\
& \lesssim\left\|Q_{Y, \zeta_{n}, r_{n}}^{*}-Q_{Y, \zeta_{0}, r_{n}}^{*}\right\|_{2, P_{Q_{0}, g^{\mathrm{ref}}}}=o_{P}(1) .
\end{aligned}
$$


Therefore, it suffices to show that $\psi_{n}^{*}-\psi_{n}^{\sim}=o_{P}(1)$ too to conclude.

Since $Q_{Y, \zeta_{n}, r_{n}}^{*} \circ r_{n}$ is a function of $W$ only, we can write

$$
\left|\psi_{n}^{*}-\psi_{n}^{\sim}\right|=\left|\left(P_{n}-P_{Q_{0}, \mathbf{g}_{n}}\right) Q_{Y, \zeta_{n}, r_{n}}^{*} \circ r_{n}\right| \leq\left\|P_{n}-P_{Q_{0}, \mathbf{g}_{n}}\right\|_{\mathcal{F}_{n}^{\prime}}
$$

where we define $\mathcal{F}_{n}^{\prime} \equiv\left\{Q_{Y, \zeta, \rho}(\epsilon) \circ \rho: \zeta \in B_{n} \times \mathcal{G}_{1, n}, \rho \in r\left(\mathcal{Q}_{1, n}\right), \epsilon \in\right.$ $\mathcal{E}\}$. By construction, $\mathcal{F}_{n}^{\prime}$ is uniformly bounded by 1 which can serve as an envelope function. Moreover, for every $\zeta_{1} \equiv\left(\beta_{1}, g_{1}\right), \zeta_{2} \equiv\left(\beta_{2}, g_{2}\right) \in B_{n} \times$ $\mathcal{G}_{1, n}, \rho_{1}, \rho_{2} \in r\left(\mathcal{Q}_{1, n}\right), \epsilon_{1}, \epsilon_{2} \in \mathcal{E}$, because (i) $\left|\left(\rho_{1}-\rho_{2}\right)(W)\right| \in\{0,1\}$, (ii) expit is 1-Lipschitz, (iii) $\mathcal{Q}_{1}$ is bounded away from 0 and 1 , logit is Lipschitz on any compact subset of $] 0,1\left[\right.$, and (iv) $\mathcal{G}_{1}$ is uniformly bounded away from 0 and 1 , the following inequalities hold pointwise:

$$
\begin{aligned}
& \left|Q_{Y, \zeta_{1}, \rho_{1}}\left(\epsilon_{1}\right) \circ \rho_{1}-Q_{Y, \zeta_{2}, \rho_{2}}\left(\epsilon_{2}\right) \circ \rho_{2}\right| \\
& =\mid\left(Q_{Y, \zeta_{1}, \rho_{1}}\left(\epsilon_{1}\right)-Q_{Y, \zeta_{2}, \rho_{2}}\left(\epsilon_{2}\right)\right) \circ \rho_{1} \\
& +\left(Q_{Y, \zeta_{2}, \rho_{2}}\left(\epsilon_{2}\right) \circ \rho_{1}-Q_{Y, \zeta_{2}, \rho_{2}}\left(\epsilon_{2}\right) \circ \rho_{2}\right) \mid \\
& \leq\left|\left(Q_{Y, \zeta_{1}, \rho_{1}}\left(\epsilon_{1}\right)-Q_{Y, \zeta_{2}, \rho_{2}}\left(\epsilon_{2}\right)\right) \circ \rho_{1}\right| \\
& +\left|\rho_{1}-\rho_{2}\right|\left|\left(Q_{Y, \zeta_{2}, \rho_{2}}\left(\epsilon_{2}\right) \circ \rho_{1}-Q_{Y, \zeta_{2}, \rho_{2}}\left(\epsilon_{2}\right) \circ \rho_{2}\right)\right| \\
& \lesssim\left|\left(Q_{Y, \beta_{1}}-Q_{Y, \beta_{2}}\right) \circ \rho_{1}\right|+\left|\epsilon_{1} / g_{1}\left(\rho_{1} \mid \cdot\right)-\epsilon_{2} / g_{2}\left(\rho_{1} \mid \cdot\right)\right|+\left|\rho_{1}-\rho_{2}\right| \\
& \lesssim\left|\left(Q_{Y, \beta_{1}}-Q_{Y, \beta_{2}}\right) \circ \rho_{1}\right|+\left|g_{1}\left(\rho_{1} \mid \cdot\right)-g_{2}\left(\rho_{1} \mid \cdot\right)\right| \\
& +\left|\epsilon_{1}-\epsilon_{2}\right|+\left|\rho_{1}-\rho_{2}\right| \\
& \leq\left|\left(Q_{Y, \beta_{1}}-Q_{Y, \beta_{2}}\right) \circ \rho_{1}\right|+\left|\left(Q_{Y, \beta_{1}}-Q_{Y, \beta_{2}}\right) \circ\left(1-\rho_{1}\right)\right| \\
& +\left|g_{1}\left(\rho_{1} \mid \cdot\right)-g_{2}\left(\rho_{1} \mid \cdot\right)\right|+\left|g_{1}\left(1-\rho_{1} \mid \cdot\right)-g_{2}\left(1-\rho_{1} \mid \cdot\right)\right| \\
& +\left|\epsilon_{1}-\epsilon_{2}\right|+\left|\rho_{1}-\rho_{2}\right| \\
& =\left|Q_{Y, \beta_{1}}-Q_{Y, \beta_{2}}\right|+\left|Q_{Y, \beta_{1}}^{-}-Q_{Y, \beta_{2}}^{-}\right| \\
& +2\left|g_{1}-g_{2}\right|+\left|\epsilon_{1}-\epsilon_{2}\right|+\left|\rho_{1}-\rho_{2}\right|
\end{aligned}
$$

where, for every $\beta \in \cup_{n \geq 1} B_{n}, Q_{Y, \beta}^{-}$is given by

$$
Q_{Y, \beta}^{-}(A, W) \equiv Q_{Y, \beta}(1-A, W) .
$$

This entails that $\mathcal{F}_{n}^{\prime}$ is separable because $\mathcal{Q}_{1, n}, \mathcal{G}_{1, n}, \mathcal{E}$ (seen as a class of constant functions with envelope function $\left.F^{\prime} \geq 1\right)$ and $r\left(\mathcal{Q}_{1, n}\right)$ are separable (the separability of $\mathcal{G}_{1, n}$ follows straightforwardly from that of $\mathcal{Q}_{1, n}$, the definition of $\mathcal{G}_{1, n}$ and continuity of $G_{n}$ ). Let $n$ be large enough so that $G_{n}=G_{\infty}$. Inequality (A.20) and the facts that (i) $\mathcal{G}_{1, n} \equiv\left\{G_{n}\left(q_{Y}\right): Q_{Y} \in\right.$ $\left.\mathcal{Q}_{1, n}\right\}=\left\{G_{\infty}\left(q_{Y}\right): Q_{Y} \in \mathcal{Q}_{1, n}\right\}$ with $G_{\infty} c_{\infty}$-Lipschitz and (ii) $\mid q_{Y, \beta_{1}}-$ $q_{Y, \beta_{2}}|\leq| Q_{Y, \beta_{1}}-Q_{Y, \beta_{2}}|+| Q_{Y, \beta_{1}}^{-}-Q_{Y, \beta_{2}}^{-} \mid$imply that

$$
J_{F^{\prime}}\left(1, \mathcal{F}_{n}^{\prime}\right) \lesssim J_{F^{\prime}}\left(1, \mathcal{Q}_{1, n}\right)+J_{F^{\prime}}\left(1, \mathcal{G}_{1, n}\right)+J_{F^{\prime}}(1, \mathcal{E})+J_{F^{\prime}}\left(1, r\left(\mathcal{Q}_{1, n}\right)\right)
$$




$$
\lesssim J_{F^{\prime}}\left(1, \mathcal{Q}_{1, n}\right)+J_{F^{\prime}}(1, \mathcal{E})+J_{F^{\prime}}\left(1, r\left(\mathcal{Q}_{1, n}\right)\right)=o(\sqrt{n})
$$

by A4. Thus, Lemma B.3 applies and Markov's inequality yields $\| P_{n}-$ $P_{Q_{0}, \mathbf{g}_{n}} \|_{\mathcal{F}_{n}^{\prime}}=o_{P}(1)$ hence $\left|\psi_{n}^{*}-\psi_{n}^{\sim}\right|=o_{P}(1)$. This completes the third step, and the proof of Proposition A.1.

The second part of Theorem 4.2 revolves around a consequence of the following result.

Proposition A.2 (first asymptotic linear expansion of $\psi_{n}^{*}$ ). Suppose that A2, A3, A4 and A4* are met. Then it holds that

$$
\psi_{n}^{*}-\psi_{r_{n}, 0}=\left(P_{n}-P_{Q_{0}, \mathbf{g}_{n}}\right)\left(d_{Y, \zeta_{0}, r_{n}}^{*}+D_{W, r_{n}}\left(Q_{\zeta_{0}, r_{n}}^{*}\right)\right)+o_{P}(1 / \sqrt{n})
$$

The asymptotic linear expansion (A.21) is obtained from the exact linear expansion stated in the next lemma.

LEMmA A.1 (exact linear expansion of $\psi_{n}^{*}$ ). It follows from the definition of $\psi_{n}^{*}$ that

$$
\begin{aligned}
\psi_{n}^{*}-\psi_{r_{n}, 0}= & -P_{Q_{0}, g_{0}} D_{r_{n}}\left(Q_{\zeta_{n}, r_{n}}^{*}, g_{0}\right) \\
= & \left(P_{n}-P_{Q_{0}, \mathbf{g}_{n}}\right)\left(d_{Y, \zeta_{0}, r_{n}}^{*}+D_{W, r_{n}}\left(Q_{\zeta_{0}, r_{n}}^{*}\right)\right) \\
& +\left(P_{n}-P_{Q_{0}, \mathbf{g}_{n}}\right)\left(\left(d_{Y, \zeta_{n}, r_{n}}^{*}-d_{Y, \zeta_{0}, r_{n}}^{*}\right)\right. \\
& \left.\quad+\left(Q_{Y, \zeta_{n}, r_{n}}^{*}-Q_{Y, \zeta_{0}, r_{n}}^{*}\right) \circ r_{n}\right)
\end{aligned}
$$

Proof of Lemma A.1. Equality (A.22) readily follows from the definitions of $D_{r_{n}}\left(Q_{\zeta_{n}, r_{n}}^{*}, g_{0}\right), \psi_{n}^{*}$ and $\psi_{r_{n}, 0}$.

We now turn to (A.23). Let us denote by $P_{n, \mathbf{g}_{n}}$ the empirical distribution of $\mathbf{O}_{n}$ weighted by $g_{n}\left(A_{i} \mid W_{i}\right) / g_{i}\left(A_{i} \mid W_{i}\right)$. By construction of the fluctuation (2.15) and definition of $\epsilon_{n}(2.16)$, it holds that $P_{n, \mathbf{g}_{n}} D_{Y, r_{n}}\left(Q_{Y, \zeta_{n}, r_{n}}^{*}, g_{n}\right)=0$. Moreover, (2.17) can be rewritten as $P_{n} D_{W, r_{n}}\left(Q_{\zeta_{n}, r_{n}}^{*}\right)=0$. Therefore, (A.22) is equivalent to

$$
\begin{aligned}
\psi_{n}^{*}-\psi_{r_{n}, 0} & =\left(P_{n}-P_{Q_{0}, g_{0}}\right) D_{W, r_{n}}\left(Q_{\zeta_{n}, r_{n}}^{*}\right) \\
& +\left(P_{n, \mathbf{g}_{n}} D_{Y, r_{n}}\left(Q_{Y, \zeta_{n}, r_{n}}^{*}, g_{n}\right)-P_{Q_{0}, g_{0}} D_{Y, r_{n}}\left(Q_{Y, \zeta_{n}, r_{n}}^{*}, g_{0}\right)\right)
\end{aligned}
$$

Adding and subtracting $\left(P_{n}-P_{Q_{0}, g_{0}}\right) D_{W, r_{n}}\left(Q_{\zeta_{0}, r_{n}}^{*}\right)$ to the first term in the RHS expression of (A.24) implies

$$
\begin{aligned}
& \left(P_{n}-P_{Q_{0}, g_{0}}\right) D_{W, r_{n}}\left(Q_{\zeta_{n}, r_{n}}^{*}\right) \\
& \quad=\left(P_{n}-P_{Q_{0}, g_{0}}\right) D_{W, r_{n}}\left(Q_{\zeta_{0}, r_{n}}^{*}\right)
\end{aligned}
$$




$$
\begin{aligned}
& +\left(P_{n}-P_{Q_{0}, g_{0}}\right)\left(D_{W, r_{n}}\left(Q_{\zeta_{n}, r_{n}}^{*}\right)-D_{W, r_{n}}\left(Q_{\zeta_{0}, r_{n}}^{*}\right)\right) \\
= & \left(P_{n}-P_{Q_{0}, g_{0}}\right) D_{W, r_{n}}\left(Q_{\zeta_{0}, r_{n}}^{*}\right) \\
& +\left(P_{n}-P_{Q_{0}, g_{0}}\right)\left(Q_{Y, \zeta_{n}, r_{n}}^{*}-Q_{Y, \zeta_{0}, r_{n}}^{*}\right) \circ r_{n} \\
= & \left(P_{n}-P_{Q_{0}, g_{n}}\right) D_{W, r_{n}}\left(Q_{\zeta_{0}, r_{n}}^{*}\right) \\
& +\left(P_{n}-P_{Q_{0}, g_{n}}\right)\left(Q_{Y, \zeta_{n}, r_{n}}^{*}-Q_{Y, \zeta_{0}, r_{n}}^{*}\right) \circ r_{n},
\end{aligned}
$$

where the last equality is valid because $\left(P_{n}-P_{Q_{0}, g_{0}}\right)$ operates on functions of $W$.

As for the second term in the RHS expression of (A.24), it equals

$$
\begin{aligned}
\frac{1}{n} \sum_{i=1}^{n}\left(\frac{g_{n}\left(A_{i} \mid W_{i}\right)}{g_{i}\left(A_{i} \mid W_{i}\right)} \frac{\mathbf{1}\left\{A_{i}=r_{n}\left(W_{i}\right)\right\}}{g_{n}\left(A_{i} \mid W_{i}\right)}\left(Y_{i}-Q_{Y, \zeta_{n}, r_{n}}^{*}\left(A_{i}, W_{i}\right)\right)\right. \\
\left.-P_{Q_{0}, g_{0}} \frac{\mathbf{1}\left\{A=r_{n}(W)\right\}}{g_{0}(A \mid W)}\left(Y-Q_{Y, \zeta_{n}, r_{n}}^{*}\right)\right) \\
=\frac{1}{n} \sum_{i=1}^{n}\left(\frac{\mathbf{1}\left\{A_{i}=r_{n}\left(W_{i}\right)\right\}}{g_{i}\left(A_{i} \mid W_{i}\right)}\left(Y_{i}-Q_{Y, \zeta_{n}, r_{n}}^{*}\left(A_{i}, W_{i}\right)\right)\right. \\
\left.-P_{Q_{0}, g_{i}} \frac{\mathbf{1}\left\{A=r_{n}(W)\right\}}{g_{i}(A \mid W)}\left(Y-Q_{Y, \zeta_{n}, r_{n}}^{*}\right)\right) \\
=\left(P_{n}-P_{Q_{0}, \mathbf{g}_{n}}\right) d_{Y, \zeta_{n}, r_{n}}^{*} \quad\left(P_{n}-P_{Q_{0}, \mathbf{g}_{n}}\right)\left(d_{Y, \zeta_{n}, r_{n}}^{*}-d_{Y, \zeta_{0}, r_{n}}^{*}\right) .
\end{aligned}
$$

The equalities (A.24), (A.25) and (A.26) imply (A.23).

We now build upon Lemma A.1 to prove Proposition A.2.

Proof of Proposition A.2. By (A.23) in Lemma A.1, (A.21) follows from

$$
\begin{aligned}
\left(P_{n}-P_{Q_{0}, \mathbf{g}_{n}}\right) & \left(\left(d_{Y, \zeta_{n}, r_{n}}^{*}-d_{Y, \zeta_{0}, r_{n}}^{*}\right)+\left(Q_{Y, \zeta_{n}, r_{n}}^{*}-Q_{Y, \zeta_{0}, r_{n}}^{*}\right) \circ r_{n}\right) \\
= & \left(P_{n}-P_{Q_{0}, \mathbf{g}_{n}}\right)\left(d_{Y, \zeta_{n}, r_{n}}^{*}-d_{Y, \zeta_{0}, r_{n}}^{*}\right) \\
+ & \left(P_{n}-P_{Q_{0}, \mathbf{g}_{n}}\right)\left(Q_{Y, \zeta_{n}, r_{n}}^{*}-Q_{Y, \zeta_{0}, r_{n}}^{*}\right) \circ r_{n}=o_{P}(1 / \sqrt{n})
\end{aligned}
$$

This is a consequence of Lemma B.4, as shown hereafter in three steps.

Step one: preliminary. We will use the following notation: for all $\beta \in B_{n}$ and $\epsilon \in \mathcal{E}$,

$$
\begin{gathered}
\Delta Q_{Y, \beta}(\epsilon) \equiv f_{r\left(Q_{Y, \beta}\right)}\left(Q_{Y, \beta}, G_{n}\left(q_{Y, \beta}\right), \epsilon\right)-f_{r\left(Q_{Y, \beta}\right)}\left(Q_{Y, \beta_{0}}, g_{0}, \epsilon_{0}\left(r\left(Q_{Y, \beta}\right)\right)\right), \\
\Delta d_{Y, \beta}(\epsilon) \equiv f_{r\left(Q_{Y, \beta}\right)}^{\prime}\left(Q_{Y, \beta}, G_{n}\left(q_{Y, \beta}\right), \epsilon\right)-f_{r\left(Q_{Y, \beta}\right)}^{\prime}\left(Q_{Y, \beta_{0}}, g_{0}, \epsilon_{0}\left(r\left(Q_{Y, \beta}\right)\right)\right),
\end{gathered}
$$


where $f_{\rho}\left(Q_{Y}, g, \epsilon\right)$ and $f_{\rho}^{\prime}\left(Q_{Y}, g, \epsilon\right)$ are respectively characterized in (A.16) and by

$$
f_{\rho}^{\prime}\left(Q_{Y}, g, \epsilon\right)(O, Z) \equiv \frac{\mathbf{1}\{A=\rho(W)\}}{Z}\left(Y-f_{\rho}\left(Q_{Y}, g, \epsilon\right)(O)\right) .
$$

The two next steps mainly consist in controlling the uniform entropy integrals of the two following sets:

$$
\begin{aligned}
\mathcal{F}_{n} & \equiv\left\{\Delta d_{Y, \beta}(\epsilon): \beta \in B_{n}, \epsilon \in \mathcal{E}\right\}, \\
\mathcal{F}_{n}^{\prime} & \equiv\left\{\Delta Q_{Y, \beta}(\epsilon) \circ r\left(Q_{Y, \beta}\right): \beta \in B_{n}, \epsilon \in \mathcal{E}\right\} .
\end{aligned}
$$

From now on, we assume that $n$ is taken large enough to ensure $\beta_{0} \in B_{n}$ and $G_{n}=G_{\infty}, \Delta d_{Y, \beta_{0}}\left(\epsilon_{0}\left(r_{0}\right)\right)=\Delta Q_{Y, \beta_{0}}\left(\epsilon_{0}\left(r_{0}\right)\right)=0$ (recall that $\left.r_{0} \equiv r\left(Q_{Y, \beta_{0}}\right)\right)$. Consequently, $0 \in \mathcal{F}_{n}$ and $0 \in \mathcal{F}_{n}^{\prime}$.

Step two: studying the first RHS term in (A.27). Since $Z$ is bounded away from 0 and 1 , the elements of $\mathcal{F}_{n}$ are uniformly bounded by a constant $c$ which can serve as an envelope function for $\mathcal{F}_{n}$. We assume without loss of generality that $c \geq \max \left(1, \sup _{\epsilon \in \mathcal{E}}|\epsilon|\right)$. Obviously, $(\boldsymbol{a})$ in Lemma B.4 is met for $\mathcal{F}_{n}$ by the resulting (constant) sequence of (constant) envelope functions. Moreover, $\Delta d_{Y, \beta_{n}}\left(\epsilon_{n}\right)-\Delta d_{Y, \beta_{0}}\left(\epsilon_{0}\left(r_{0}\right)\right)=\Delta d_{Y, \beta_{n}}\left(\epsilon_{n}\right)=d_{Y, \zeta_{n}, r_{n}}^{*}-d_{Y, \zeta_{0}, r_{n}}^{*}$ satisfies

$$
\begin{aligned}
\mid\left(\Delta d_{Y, \beta_{n}}\left(\epsilon_{n}\right)-\right. & \left.\Delta d_{Y, \beta_{0}}\left(\epsilon_{0}\left(r_{0}\right)\right)\right)(O, Z) \mid \\
& =\left|\frac{\mathbf{1}\left\{A=r_{n}(W)\right\}}{Z}\left(Q_{Y, \zeta_{n}, r_{n}}^{*}(A, W)-Q_{Y, \zeta_{0}, r_{n}}^{*}(A, W)\right)\right| \\
& \lesssim\left|Q_{Y, \zeta_{n}, r_{n}}^{*}(A, W)-Q_{Y, \zeta_{0}, r_{n}}^{*}(A, W)\right|
\end{aligned}
$$

hence the convergence $\left\|\Delta d_{Y, \beta_{n}}\left(\epsilon_{n}\right)-\Delta d_{Y, \beta_{0}}\left(\epsilon_{0}\left(r_{0}\right)\right)\right\|_{2, P_{Q_{0}}, g^{\mathrm{ref}}}=o_{P}(1)$ follows from the second step of the proof of Proposition A.1, whose assumptions are met.

It remains to prove that $\mathcal{F}_{n}$ is separable and satisfies (b) in Lemma B.4. Set arbitrarily $\left(\beta_{1}, \epsilon_{1}\right),\left(\beta_{2}, \epsilon_{2}\right) \in B_{n} \times \mathcal{E}$ and define $g_{1} \equiv G_{n}\left(q_{Y, \beta_{1}}\right), g_{2} \equiv$ $G_{n}\left(q_{Y, \beta_{2}}\right), \rho_{1} \equiv r\left(Q_{Y, \beta_{1}}\right)$ and $\rho_{2} \equiv r\left(Q_{Y, \beta_{2}}\right)$. First, we note that

$$
\begin{aligned}
\left|\left(\Delta d_{Y, \beta_{1}}\left(\epsilon_{1}\right)-\Delta d_{Y, \beta_{2}}\left(\epsilon_{2}\right)\right)(O, Z)\right| \\
=\left|\frac{\mathbf{1}\left\{A=\rho_{1}(W)\right\}}{Z} \Delta Q_{Y, \beta_{1}}\left(\epsilon_{1}\right)(O)-\frac{\mathbf{1}\left\{A=\rho_{2}(W)\right\}}{Z} \Delta Q_{Y, \beta_{2}}\left(\epsilon_{2}\right)(O)\right| \\
\lesssim \mid \mathbf{1}\left\{A=\rho_{1}(W)\right\}\left(\Delta Q_{Y, \beta_{1}}\left(\epsilon_{1}\right)(O)-\Delta Q_{Y, \beta_{2}}\left(\epsilon_{2}\right)(O)\right) \\
\quad+\left|\left(\mathbf{1}\left\{A=\rho_{1}(W)\right\}-\mathbf{1}\left\{A=\rho_{2}(W)\right\}\right) \Delta Q_{Y, \beta_{2}}\left(\epsilon_{2}\right)(O)\right|,
\end{aligned}
$$


which yields the pointwise inequality

$$
\left|\Delta d_{Y, \beta_{1}}\left(\epsilon_{1}\right)-\Delta d_{Y, \beta_{2}}\left(\epsilon_{2}\right)\right| \lesssim\left|\Delta Q_{Y, \beta_{1}}\left(\epsilon_{1}\right)-\Delta Q_{Y, \beta_{2}}\left(\epsilon_{2}\right)\right|+\left|\rho_{1}-\rho_{2}\right| .
$$

Second, we focus on the left RHS term in (A.29). It holds pointwise that

$$
\begin{aligned}
& \left|\Delta Q_{Y, \beta_{1}}\left(\epsilon_{1}\right)-\Delta Q_{Y, \beta_{2}}\left(\epsilon_{2}\right)\right| \\
& \leq \mid \\
& \quad+f_{\rho_{1}}\left(Q_{Y, \beta_{1}}, g_{1}, \epsilon_{1}\right)-f_{\rho_{1}}\left(Q_{Y, \beta_{2}}, g_{2}, \epsilon_{2}\right) \mid \\
& \quad+\left|f_{\rho_{1}}\left(Q_{Y, \beta_{0}}, g_{0}, \epsilon_{0}\left(\rho_{1}\right)\right)-f_{\rho_{1}}\left(Q_{Y, \beta_{0}}, g_{0}, \epsilon_{0}\left(\rho_{2}\right)\right)\right| \\
& \quad+\left|f_{\rho_{1}}\left(Q_{Y, \beta_{2}}, g_{2}, \epsilon_{2}\right)-f_{\rho_{2}}\left(Q_{Y, \beta_{2}}, g_{2}, \epsilon_{2}\right)\right| \\
& \quad+\left|f_{\rho_{1}}\left(Q_{Y, \beta_{0}}, g_{0}, \epsilon_{0}\left(\rho_{2}\right)\right)-f_{\rho_{2}}\left(Q_{Y, \beta_{0}}, g_{0}, \epsilon_{0}\left(\rho_{2}\right)\right)\right| .
\end{aligned}
$$

For the same reasons as those which lead to (A.17) and because $G_{n} c_{\infty^{-}}$ Lipschitz implies $\left|g_{1}-g_{2}\right| \lesssim\left|q_{Y, \beta_{1}}-q_{Y, \beta_{2}}\right| \leq\left|Q_{Y, \beta_{1}}-Q_{Y, \beta_{2}}\right|+\left|Q_{Y, \beta_{1}}^{-}-Q_{Y, \beta_{2}}^{-}\right|$, we derive the following pointwise inequalities from the previous one:

$$
\begin{aligned}
\mid \Delta Q_{Y, \beta_{1}}\left(\epsilon_{1}\right)- & \Delta Q_{Y, \beta_{2}}\left(\epsilon_{2}\right) \mid \\
\lesssim & \left|Q_{Y, \beta_{1}}-Q_{Y, \beta_{2}}\right|+\left|g_{1}-g_{2}\right| \\
& +\left|\epsilon_{1}-\epsilon_{2}\right|+\left|\epsilon_{0}\left(\rho_{1}\right)-\epsilon_{0}\left(\rho_{2}\right)\right| \\
& +\left|H_{\rho_{1}}\left(g_{2}\right)-H_{\rho_{2}}\left(g_{2}\right)\right|+\left|H_{\rho_{1}}\left(g_{0}\right)-H_{\rho_{2}}\left(g_{0}\right)\right| \\
\lesssim & \left|Q_{Y, \beta_{1}}-Q_{Y, \beta_{2}}\right|+\left|Q_{Y, \beta_{1}}^{-}-Q_{Y, \beta_{2}}^{-}\right| \\
& +\left|\epsilon_{1}-\epsilon_{2}\right|+\left|\epsilon_{0}\left(\rho_{1}\right)-\epsilon_{0}\left(\rho_{2}\right)\right| \\
& +\left|H_{\rho_{1}}\left(g_{2}\right)-H_{\rho_{2}}\left(g_{2}\right)\right|+\left|H_{\rho_{1}}\left(g_{0}\right)-H_{\rho_{2}}\left(g_{0}\right)\right| .
\end{aligned}
$$

Consider the last term in the above RHS sum. Because $\mathcal{G}_{1}$ is uniformly bounded away from 0 and 1 , we have $\left|H_{\rho_{1}}\left(g_{0}\right)(O)-H_{\rho_{2}}\left(g_{0}\right)(O)\right| \lesssim \mid \mathbf{1}\{A=$ $\rho_{1}(W)-\mathbf{1}\left\{A=\rho_{2}(W)\right\}|=| \rho_{1}(W)-\rho_{2}(W) \mid$ (we already used this argument to derive (A.13) in the first step of the proof of Proposition A.1). The last but one term is dealt with similarly. It remains to control the most delicate term, $\left|\epsilon_{0}\left(\rho_{1}\right)-\epsilon_{0}\left(\rho_{2}\right)\right|$. Let $z_{1}, z_{2}$ be characterized over $\mathcal{E}$ by $z_{j}(\epsilon) \equiv P_{Q_{0}, g_{0}} D_{Y, \rho_{j}}\left(Q_{Y, \zeta_{0}, \rho_{j}}(\epsilon), g_{0}\right)=P_{Q_{0}, g_{0}} f_{\rho_{j}, \epsilon}(j=1,2$; see (A.11) for the definition of $\left.f_{\rho, \epsilon}\right)$. For the same reasons as in the first step of the proof of Proposition A.1 (substitute $\rho_{1}$ or $\rho_{2}$ for $\left.r_{n}\right), z_{1}\left(\epsilon_{0}\left(\rho_{1}\right)\right)=$ $z_{2}\left(\epsilon_{0}\left(\rho_{2}\right)\right)=0$ and $\left|\epsilon-\epsilon_{0}\left(\rho_{2}\right)\right| \lesssim\left|z_{2}(\epsilon)\right|$ for all $\epsilon \in \mathcal{E}$. Moreover, by (A.13), $\left|z_{1}(\epsilon)-z_{2}(\epsilon)\right| \lesssim\left\|\rho_{1}-\rho_{2}\right\|_{2, P_{Q_{0}, g^{\text {ref }}}}$ for all $\epsilon \in \mathcal{E}$, hence in particular

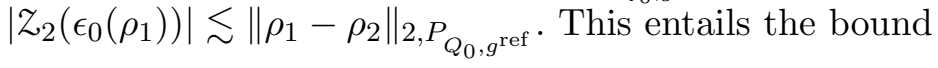

$$
\left|\epsilon_{0}\left(\rho_{1}\right)-\epsilon_{0}\left(\rho_{2}\right)\right| \lesssim\left\|\rho_{1}-\rho_{2}\right\|_{2, P_{Q_{0}, g^{\mathrm{ref}}}} .
$$

Consequently, (A.31) implies the pointwise inequality

$$
\left|\Delta Q_{Y, \beta_{1}}\left(\epsilon_{1}\right)-\Delta Q_{Y, \beta_{2}}\left(\epsilon_{2}\right)\right| \lesssim\left|Q_{Y, \beta_{1}}-Q_{Y, \beta_{2}}\right|+\left|Q_{Y, \beta_{1}}^{-}-Q_{Y, \beta_{2}}^{-}\right|
$$




$$
+\left|\rho_{1}-\rho_{2}\right|+\left\|\rho_{1}-\rho_{2}\right\|_{2, P_{Q_{0}, g} \text { ref }}+\left|\epsilon_{1}-\epsilon_{2}\right| .
$$

Combining (A.29) and (A.33) finally yields (with the same notation as in (A.20))

$$
\begin{array}{ll}
\left|\Delta d_{Y, \beta_{1}}\left(\epsilon_{1}\right)-\Delta d_{Y, \beta_{2}}\left(\epsilon_{2}\right)\right| \lesssim & \left|Q_{Y, \beta_{1}}-Q_{Y, \beta_{2}}\right|+\left|Q_{Y, \beta_{1}}^{-}-Q_{Y, \beta_{2}}^{-}\right| \\
& +\left|\rho_{1}-\rho_{2}\right|+\left\|\rho_{1}-\rho_{2}\right\|_{2, P_{Q_{0}, g} \text { ref }}+\left|\epsilon_{1}-\epsilon_{2}\right| .
\end{array}
$$

Inequality (A.34) entails that $\mathcal{F}_{n}$ is separable because $\mathcal{Q}_{1, n}, r\left(\mathcal{Q}_{1, n}\right)$ and $\mathcal{E}$ (seen as a class of constant functions with constant envelope function $c^{\prime}$ ) are separable. Moreover, since the definition of the uniform entropy integral involve a supremum over probability measures, (A.15) and (A.34) also imply that, for each $\delta>0$,

$$
J_{c}\left(\delta, \mathcal{F}_{n}\right) \lesssim J_{c}\left(\delta, \mathcal{Q}_{1, n}\right)+J_{c}\left(\delta, r\left(\mathcal{G}_{1, n}\right)\right)+J_{c}(\delta, \mathcal{E}) .
$$

Consequently, A4* guarantees that (b) in Lemma B.4 is met. Thus, Lemma B.4 applies and yields

$\sqrt{n}\left(P_{n}-P_{Q_{0}, \mathbf{g}_{n}}\right)\left(d_{Y, \zeta_{n}, r_{n}}^{*}-d_{Y, \zeta_{0}, r_{n}}^{*}\right)=\sqrt{n}\left(P_{n}-P_{Q_{0}, \mathbf{g}_{n}}\right) \Delta d_{Y, \beta_{n}}\left(\epsilon_{n}\right)=o_{P}(1)$.

Step three: studying the second RHS term in (A.27). The elements of $\mathcal{F}_{n}^{\prime}$ are uniformly bounded by 1 hence by a constant $c^{\prime} \geq \max \left(1, \sup _{\epsilon \in \mathcal{E}}|\epsilon|\right)$ which can serve as an envelope function for $\mathcal{F}_{n}^{\prime}$. Obviously, $(\boldsymbol{a})$ in Lemma B.4 is met for $\mathcal{F}_{n}^{\prime}$ by the resulting (constant) sequence of (constant) envelope functions. Moreover, (A.20) implies that

$$
\begin{aligned}
\Delta Q_{Y, \beta_{n}}\left(\epsilon_{n}\right) \circ r\left(Q_{Y, \beta_{n}}\right)- & \Delta Q_{Y, \beta_{0}}\left(\epsilon_{0}\left(r_{0}\right)\right) \circ r\left(Q_{Y, \beta_{0}}\right) \\
& =\Delta Q_{Y, \beta_{n}}\left(\epsilon_{n}\right) \circ r_{n} \\
& =\left(Q_{Y, \zeta_{n}, r_{n}}\left(\epsilon_{n}\right)-Q_{Y, \zeta_{0}, r_{n}}\left(\varepsilon_{0}\left(r_{n}\right)\right)\right) \circ r_{n} \\
& =\left(Q_{Y, \zeta_{n}, r_{n}}^{*}-Q_{Y, \zeta_{0}, r_{n}}^{*}\right) \circ r_{n}
\end{aligned}
$$

satisfies (with the same notational convention)

$$
\begin{aligned}
& \left|\Delta Q_{Y, \beta_{n}}\left(\epsilon_{n}\right) \circ r\left(Q_{Y, \beta_{n}}\right)-\Delta Q_{Y, \beta_{0}}\left(\epsilon_{0}\left(r_{0}\right)\right) \circ r\left(Q_{Y, \beta_{0}}\right)\right| \\
& \quad \lesssim\left|Q_{Y, \beta_{n}}-Q_{Y, \beta_{0}}\right|+\left|Q_{Y, \beta_{n}}^{-}-Q_{Y, \beta_{0}}^{-}\right|+\left|g_{n}-g_{0}\right|+\left|\epsilon_{n}-\epsilon_{0}\left(r_{n}\right)\right| .
\end{aligned}
$$

Because $g^{\text {ref }}$ is bounded away from 0 and 1 , this yields

$$
\begin{aligned}
& \left\|\Delta Q_{Y, \beta_{n}}\left(\epsilon_{n}\right) \circ r\left(Q_{Y, \beta_{n}}\right)-\Delta Q_{Y, \beta_{0}}\left(\epsilon_{0}\left(r_{0}\right)\right) \circ r\left(Q_{Y, \beta_{0}}\right)\right\|_{2, P_{Q_{0}, g^{\mathrm{ref}}}} \\
& \lesssim\left\|Q_{Y, \beta_{n}}-Q_{Y, \beta_{0}}\right\|_{2, P_{Q_{0}, g^{\mathrm{ref}}}}+\left\|g_{n}-g_{0}\right\|_{2, P_{Q_{0}, g^{\mathrm{ref}}}}+\left|\epsilon_{n}-\epsilon_{0}\left(r_{n}\right)\right|=o_{P}(1)
\end{aligned}
$$


because each term in the above RHS sum is $o_{P}(1)$ by Proposition 4.1, the first step of the proof of Proposition A.1 and (A.15).

It remains to prove that $\mathcal{F}_{n}^{\prime}$ is separable and satisfies (b) in Lemma B.4. For this, set arbitrarily $\left(\beta_{1}, \epsilon_{1}\right),\left(\beta_{2}, \epsilon_{2}\right) \in B_{n} \times \mathcal{E}$, define $g_{1} \equiv G_{n}\left(q_{Y, \beta_{1}}\right)$, $g_{2} \equiv G_{n}\left(q_{Y, \beta_{2}}\right), \rho_{1} \equiv r\left(Q_{Y, \beta_{1}}\right)$ and $\rho_{2} \equiv r\left(Q_{Y, \beta_{2}}\right)$, then note that

$$
\begin{aligned}
\mid \Delta Q_{Y, \beta_{1}}\left(\epsilon_{1}\right) \circ \rho_{1}- & \Delta Q_{Y, \beta_{2}}\left(\epsilon_{2}\right) \circ \rho_{2} \mid \\
\leq\left|\Delta Q_{Y, \beta_{1}}\left(\epsilon_{1}\right) \circ \rho_{1}-\Delta Q_{Y, \beta_{1}}\left(\epsilon_{1}\right) \circ \rho_{2}\right| & +\left|\left(\Delta Q_{Y, \beta_{1}}\left(\epsilon_{1}\right)-\Delta Q_{Y, \beta_{2}}\left(\epsilon_{2}\right)\right) \circ \rho_{2}\right| .
\end{aligned}
$$

Consider the first term in the above RHS sum. Because (i) it equals zero when $\rho_{1}$ and $\rho_{2}$ coincide, (ii) $\left|\rho_{1}-\rho_{2}\right| \in\{0,1\}$, and (iii) $\mid \Delta Q_{Y, \beta_{1}}\left(\epsilon_{1}\right)-$ $\Delta Q_{Y, \beta_{1}}\left(\epsilon_{1}\right) \mid \leq 2$, we see that it is actually upper-bounded by $2\left|\rho_{1}-\rho_{2}\right|$. We now turn to the second term. By (A.33), it satisfies the following pointwise inequalities:

$$
\begin{aligned}
\left|\left(\Delta Q_{Y, \beta_{1}}\left(\epsilon_{1}\right)-\Delta Q_{Y, \beta_{2}}\left(\epsilon_{2}\right)\right) \circ \rho_{2}\right| \lesssim\left|Q_{Y, \beta_{1}}-Q_{Y, \beta_{2}}\right|+\left|Q_{Y, \beta_{1}}^{-}-Q_{Y, \beta_{2}}^{-}\right| \\
+\left|\rho_{1}-\rho_{2}\right|+|| \rho_{1}-\rho_{2}||_{2, P_{Q_{0}, g^{\mathrm{ref}}}}+\left|\epsilon_{1}-\epsilon_{2}\right| .
\end{aligned}
$$

We thus have

$$
\begin{aligned}
\left|\Delta Q_{Y, \beta_{1}}\left(\epsilon_{1}\right) \circ \rho_{1}-\Delta Q_{Y, \beta_{2}}\left(\epsilon_{2}\right) \circ \rho_{2}\right| \lesssim\left|Q_{Y, \beta_{1}}-Q_{Y, \beta_{2}}\right| \\
\quad+\left|Q_{Y, \beta_{1}}^{-}-Q_{Y, \beta_{2}}^{-}\right|+\left|\rho_{1}-\rho_{2}\right|+\left\|\rho_{1}-\rho_{2}\right\|_{2, P_{Q_{0}, g^{\mathrm{ref}}}}+\left|\epsilon_{1}-\epsilon_{2}\right| .
\end{aligned}
$$

As argued in the previous step, the above pointwise inequality yields that $\mathcal{F}_{n}^{\prime}$ is separable and that, for each $\delta>0$,

$$
J_{c^{\prime}}\left(\delta, \mathcal{F}_{n}^{\prime}\right) \lesssim J_{c^{\prime}}\left(\delta, \mathcal{Q}_{1, n}\right)+J_{c^{\prime}}\left(\delta, r\left(\mathcal{G}_{1, n}\right)\right)+J_{c^{\prime}}(\delta, \mathcal{E}) .
$$

Consequently, A4* guarantees that $(\boldsymbol{b})$ in Lemma B.4 is met. Thus, Lemma B.4 applies and implies $\sqrt{n}\left(P_{n}-P_{Q_{0}, \mathbf{g}_{n}}\right)\left(Q_{Y, \zeta_{n}, r_{n}}^{*}-Q_{Y, \zeta_{0}, r_{n}}^{*}\right) \circ r_{n}=$ $\sqrt{n}\left(P_{n}-P_{Q_{0}, \mathbf{g}_{n}}\right) \Delta Q_{Y, \beta_{n}}\left(\epsilon_{n}\right) \circ r\left(Q_{Y, \beta_{n}}\right)=o_{P}(1)$. Combining the conclusions of steps two and three shows that (A.27) holds, and therefore completes the proof.

Proposition A.2 has the following corollary. Proving it will complete the proof of Theorem 4.2 .

COROllary A.1 (second asymptotic linear expansion of $\psi_{n}^{*}$ and resulting central limit theorem). Suppose that A1, A2, A3, A4, A4*, and $\mathbf{A 5}$ are met. Then (4.15) holds. Moreover, $\Sigma_{n}=\Sigma_{0}+o_{P}(1)$ with $\Sigma_{0}>0$ and $\sqrt{n / \Sigma_{n}}\left(\psi_{n}^{*}-\psi_{0, r_{n}}\right)$ converges in law to the standard normal distribution. 
Proof of Corollary A.1. This is a four-part proof.

Step one: preliminary. Recall (4.9), (4.10), (4.12), (4.13), (A.3), (A.4) and set

$$
\begin{aligned}
f_{0} & \equiv d_{W, 0}^{*}+E_{Q_{W, 0}}\left(Q_{Y, \zeta_{0}, r_{0}}^{*} \circ r_{0}(W)\right)+d_{Y, 0}^{*}=Q_{Y, \zeta_{0}, r_{0}}^{*} \circ r_{0}+d_{Y, 0}^{*}, \\
f_{n} & \equiv d_{W, n}^{*}+\psi_{n}^{*}+d_{Y, n}^{*}=Q_{Y, \zeta_{n}, r_{n}}^{*} \circ r_{n}+d_{Y, n}^{*}, \\
f_{0, n} & \equiv Q_{Y, \zeta_{0}, r_{n}}^{*} \circ r_{n}+d_{Y, \zeta_{0}, r_{n}}^{*} .
\end{aligned}
$$

A straightforward adaptation of the argument leading to (A.18) in step three of the proof of Proposition A.1 also yields $E_{Q_{W, 0}}\left(Q_{Y, \zeta_{0}, r_{0}}^{*} \circ r_{0}(W)\right)=\psi_{0}$. It is then apparent that $P_{n}\left(f_{n}-\psi_{n}^{*}\right)=P_{Q_{0}, g_{0}}\left(f_{0}-\psi_{0}\right)=0$. Now, note that $\Sigma_{0}, \Sigma_{n}$ defined in (4.11) and (4.14) can be rewritten

$$
\begin{aligned}
& \Sigma_{0}=P_{Q_{0}, g_{0}}\left(f_{0}-\psi_{0}\right)^{2}=P_{Q_{0}, g_{0}} f_{0}^{2}-\psi_{0}^{2}, \\
& \Sigma_{n}=P_{n}\left(f_{n}-\psi_{n}^{*}\right)^{2}=P_{n} f_{n}^{2}-\psi_{n}^{* 2},
\end{aligned}
$$

and that $\Sigma_{0}>0$ by $\mathbf{A} \mathbf{1}$. Introduce also $S_{n} \equiv P_{Q_{0}, \mathbf{g}_{n}}\left(f_{0}-\psi_{0}\right)^{2}$.

For each $(f, \zeta, r, \psi)$ among $\left(f_{0}, \zeta_{0}, r_{0}, 0\right)$ or $\left(f_{0}, \zeta_{0}, r_{0}, \psi_{0}\right)$ or $\left(f_{n}, \zeta_{n}, r_{n}, 0\right)$ or $\left(f_{n}, \zeta_{n}, r_{n}, \psi_{n}^{*}\right)$, it holds that

$$
\begin{aligned}
& P_{Q_{0}, \mathbf{g}_{n}}(f-\psi)^{2} \\
&=\frac{1}{n} \sum_{i=1}^{n} P_{Q_{0}, g_{i}}(f-\psi)^{2} \\
&=P_{Q_{0}, g_{0}}\left(\left(Q_{Y, \zeta, r}^{*} \circ r-\psi\right)^{2}+2\left(Q_{Y, \zeta, r}^{*} \circ r-\psi\right) D_{Y, r}\left(Q_{\zeta, r}^{*}, g_{0}\right)\right) \\
& \quad+\frac{1}{n} \sum_{i=1}^{n} P_{Q_{0}, g_{0}} \frac{\mathbf{1}\{A=r(W)\}}{g_{0} g_{i}}\left(Y-Q_{Y, \zeta, r}^{*}\right)^{2} \\
&=P_{Q_{0}, g_{0}}\left(\left(Q_{Y, \zeta, r}^{*} \circ r-\psi\right)^{2}+2\left(Q_{Y, \zeta, r}^{*} \circ r-\psi\right) D_{Y, r}\left(Q_{Y, \zeta, r}^{*}, g_{0}\right)\right) \\
& \quad+P_{Q_{0}, g_{0}} \frac{\mathbf{1}\{A=r(W)\}}{g_{0}}\left(Y-Q_{Y, \zeta, r}^{*}\right)^{2} \times \frac{1}{n} \sum_{i=1}^{n} \frac{1}{g_{i}}
\end{aligned}
$$

and, similarly,

$$
\begin{aligned}
& P_{Q_{0}, g_{0}}(f-\psi)^{2} \\
& =P_{Q_{0}, g_{0}}\left(\left(Q_{Y, \zeta, r}^{*} \circ r-\psi\right)^{2}+2\left(Q_{Y, \zeta, r}^{*} \circ r-\psi\right) D_{Y, r}\left(Q_{Y, \zeta, r}^{*}, g_{0}\right)\right) \\
& \quad+P_{Q_{0}, g_{0}} \frac{\mathbf{1}\{A=r(W)\}}{g_{0}}\left(Y-Q_{Y, \zeta, r}^{*}\right)^{2} \times \frac{1}{g_{0}} .
\end{aligned}
$$

Since $\left(Y-Q_{Y, \zeta, r}^{*}\right)^{2} \leq 1$ and because $g_{0}, g^{\text {ref }}$ and all $g_{i} \mathrm{~s}(i \geq 1)$ are bounded away from 0 and 1, applying the Cauchy-Schwarz inequality then yields

$$
\left|P_{Q_{0}, \mathbf{g}_{n}}(f-\psi)^{2}-P_{Q_{0}, g_{0}}(f-\psi)^{2}\right|
$$




$$
\begin{aligned}
& =\left|P_{Q_{0}, g_{0}} \frac{\mathbf{1}\{A=r(W)\}}{g_{0}}\left(Y-Q_{Y, \zeta, r}^{*}\right)^{2}\left(\frac{1}{n} \sum_{i=1}^{n} \frac{1}{g_{i}}-\frac{1}{g_{0}}\right)\right| \\
& \lesssim P_{Q_{0}, g_{0}}\left|\frac{1}{n} \sum_{i=1}^{n} \frac{1}{g_{i}}-\frac{1}{g_{0}}\right| \lesssim\left\|\frac{1}{n} \sum_{i=1}^{n}\left(g_{i}-g_{0}\right)\right\|_{2, P_{Q_{0}, g} \mathrm{ref}} \cdot
\end{aligned}
$$

Step two: studying $\Sigma_{n}$ and $\Sigma_{0}$. By Lemma A.2 (presented after this proof), (A.35) teaches us that $E\left(S_{n}\right)=\Sigma_{0}+o(1)$ and $S_{n}=\Sigma_{0}+o_{P}(1)$ (take $(f, \psi)=\left(f_{0}, \psi_{0}\right)$ in $($ A.35 $\left.)\right)$.

Let us show now that $\Sigma_{n}=\Sigma_{0}+o_{P}(1)$ by proving $\Sigma_{n}-S_{n}+\left(\psi_{n}^{* 2}-\psi_{0}^{2}\right)=$ $\Sigma_{n}-S_{n}+o_{P}(1)=o_{P}(1)$. We use the following decomposition:

$$
\begin{aligned}
\Sigma_{n}-S_{n}+\left(\psi_{n}^{* 2}-\psi_{0}^{2}\right) & =P_{n} f_{n}^{2}-P_{Q_{0}, \mathbf{g}_{n}} f_{0}^{2} \\
& =\left(P_{n}-P_{Q_{0}, \mathbf{g}_{n}}\right) f_{n}^{2}+P_{Q_{0}, \mathbf{g}_{n}}\left(f_{n}^{2}-f_{0}^{2}\right) \\
& =\left(P_{n}-P_{Q_{0}, \mathbf{g}_{n}}\right) f_{n}^{2}+P_{Q_{0}, g_{0}}\left(f_{n}^{2}-f_{0}^{2}\right)+o_{P}(1),
\end{aligned}
$$

where the last equality holds because $P_{Q_{0}, \mathbf{g}_{n}} f^{2}=P_{Q_{0}, g_{0}} f^{2}+o_{P}(1)$ for both $f=f_{0}$ and $f=f_{n}$ by (A.35) (take $(f, \psi)=\left(f_{0}, 0\right)$ and $\left.(f, \psi)=\left(f_{n}, 0\right)\right)$ and Lemma A.2. Let us consider in turn the two RHS terms in (A.36).

- From now on, we assume that $n$ is taken large enough to ensure $G_{n}=$ $G_{\infty}$. For all $\beta \in B_{n}$ and $\epsilon \in \mathcal{E}$, let

$$
d_{Y, \beta}(\epsilon) \equiv f_{r\left(Q_{Y, \beta}\right)}^{\prime}\left(Q_{Y, \beta}, G_{n}\left(q_{Y, \beta}\right), \epsilon\right)
$$

where $f_{\rho}^{\prime}$ is defined in (A.28). Introduce

$$
\begin{aligned}
\mathcal{F}_{n} \equiv\left\{Q_{Y, \zeta, \rho}(\epsilon) \circ \rho+d_{Y, \beta}(\epsilon): \beta \in B_{n},\right. & \\
& \left.g=G_{n}\left(q_{Y, \beta}\right), \zeta=(\beta, g), \rho=r\left(Q_{Y, \beta}\right), \epsilon \in \mathcal{E}\right\} .
\end{aligned}
$$

In particular, $f_{n}^{2}=\left(Q_{Y, \zeta_{n}, r_{n}}\left(\epsilon_{n}\right) \circ r_{n}+d_{Y, \beta_{n}}\left(\epsilon_{n}\right)\right)^{2}$ belongs to $\left(\mathcal{F}_{n}\right)^{2} \equiv$ $\left\{f^{2}: f \in \mathcal{F}_{n}\right\}$. The following upper-bound motivates the definition of $\mathcal{F}_{n}$ :

$$
\left|\left(P_{n}-P_{Q_{0}, \mathbf{g}_{n}}\right) f_{n}^{2}\right| \leq\left\|P_{n}-P_{Q_{0}, \mathbf{g}_{n}}\right\|_{\left(\mathcal{F}_{n}\right)^{2}} .
$$

If $\left\|P_{n}-P_{Q_{0}, \mathbf{g}_{n}}\right\|_{\left(\mathcal{F}_{n}\right)^{2}}=o_{P}(1)$ then $\left(P_{n}-P_{Q_{0}, \mathbf{g}_{n}}\right) f_{n}^{2}=o_{P}(1)$ too. We prove the former convergence by applying Lemma B.3 and Markov's inequality.

Since $\mathcal{F}_{n}$ is uniformly bounded, there exists a constant $c$ larger than $\max \left(1, \sup _{\epsilon \in \mathcal{E}}|\epsilon|\right)$ which can serve as an envelope function to both $\mathcal{F}_{n}$ and $\left(\mathcal{F}_{n}\right)^{2}$. Set arbitrarily $\left(\beta_{1}, \epsilon_{1}\right),\left(\beta_{2}, \epsilon_{2}\right) \in B_{n} \times \mathcal{E}$, define $g_{1}=$ $G_{n}\left(q_{Y, \beta_{1}}\right), g_{2}=G_{n}\left(q_{Y, \beta_{2}}\right), \zeta_{1}=\left(\beta_{1}, g_{1}\right), \zeta_{2}=\left(\beta_{2}, g_{2}\right), \rho_{1}=r\left(Q_{Y, \beta_{1}}\right)$, 
$\rho_{2}=r\left(Q_{Y, \beta_{2}}\right)$, let $f_{1} \equiv Q_{Y, \zeta_{1}, \rho_{1}}\left(\epsilon_{1}\right) \circ \rho_{1}+d_{Y, \beta_{1}}\left(\epsilon_{1}\right), f_{2} \equiv Q_{Y, \zeta_{2}, \rho_{2}}\left(\epsilon_{2}\right) \circ$ $\rho_{2}+d_{Y, \beta_{2}}\left(\epsilon_{2}\right)$. Because $\left|f_{1}^{2}-f_{2}^{2}\right| \lesssim\left|f_{1}-f_{2}\right|$, it holds that $J_{c}\left(1,\left(\mathcal{F}_{n}\right)^{2}\right) \lesssim$ $J_{c}\left(1, \mathcal{F}_{n}\right)$ and the separability of $\mathcal{F}_{n}$ implies that of $\left(\mathcal{F}_{n}\right)^{2}$. So, we now focus on $\mathcal{F}_{n}$.

Obviously, $\left|f_{1}-f_{2}\right| \leq\left|Q_{Y, \zeta_{1}, \rho_{1}}\left(\epsilon_{1}\right) \circ \rho_{1}-Q_{Y, \zeta_{2}, \rho_{2}}\left(\epsilon_{2}\right) \circ \rho_{2}\right|+\mid d_{Y, \beta_{1}}\left(\epsilon_{1}\right)-$ $d_{Y, \beta_{2}}\left(\epsilon_{2}\right) \mid$. The first RHS is controlled in (A.20). We deal with the second one in the same spirit as in step two of the proof of Proposition A.2. First,

$$
\begin{aligned}
& \left|\left(d_{Y, \beta_{1}}\left(\epsilon_{1}\right)-d_{Y, \beta_{2}}\left(\epsilon_{2}\right)\right)(O, Z)\right| \\
& =\mid \frac{\mathbf{1}\left\{A=\rho_{1}(W)\right\}}{Z}\left(Y-f_{\rho_{1}}\left(Q_{Y, \beta_{1}}, g_{1}, \epsilon_{1}\right)(O)\right) \\
& \quad-\frac{\mathbf{1}\left\{A=\rho_{2}(W)\right\}}{Z}\left(Y-f_{\rho_{2}}\left(Q_{Y, \beta_{2}}, g_{2}, \epsilon_{2}\right)(O)\right) \mid \\
& \lesssim\left|\mathbf{1}\left\{A=\rho_{1}(W)\right\}\left(f_{\rho_{1}}\left(Q_{Y, \beta_{1}}, g_{1}, \epsilon_{1}\right)(O)-f_{\rho_{2}}\left(Q_{Y, \beta_{2}}, g_{2}, \epsilon_{2}\right)(O)\right)\right| \\
& \quad+\left|\left(\mathbf{1}\left\{A=\rho_{1}(W)\right\}-\mathbf{1}\left\{A=\rho_{2}(W)\right\}\right) f_{\rho_{2}}\left(Q_{Y, \beta_{2}}, g_{2}, \epsilon_{2}\right)(O)\right|
\end{aligned}
$$

which yields

$\left|d_{Y, \beta_{1}}\left(\epsilon_{1}\right)-d_{Y, \beta_{2}}\left(\epsilon_{2}\right)\right| \lesssim\left|f_{\rho_{1}}\left(Q_{Y, \beta_{1}}, g_{1}, \epsilon_{1}\right)-f_{\rho_{2}}\left(Q_{Y, \beta_{2}}, g_{2}, \epsilon_{2}\right)\right|+\left|\rho_{1}-\rho_{2}\right|$.

Second, the previous pointwise inequality implies

$\left|d_{Y, \beta_{1}}\left(\epsilon_{1}\right)-d_{Y, \beta_{2}}\left(\epsilon_{2}\right)\right| \lesssim\left|Q_{Y, \beta_{1}}-Q_{Y, \beta_{2}}\right|+\left|g_{1}-g_{2}\right|+\left|\epsilon_{1}-\epsilon_{2}\right|+\left|\rho_{1}-\rho_{2}\right|$.

In summary,

$$
\begin{aligned}
\left|f_{1}-f_{2}\right| \lesssim\left|Q_{Y, \beta_{1}}-Q_{Y, \beta_{2}}\right| & +\left|Q_{Y, \beta_{1}}^{-}-Q_{Y, \beta_{2}}^{-}\right| \\
& +\left|g_{1}-g_{2}\right|+\left|\epsilon_{1}-\epsilon_{2}\right|+\left|\rho_{1}-\rho_{2}\right| .
\end{aligned}
$$

Since $\mathcal{Q}_{1, n}$ hence $\mathcal{G}_{1, n}$ (already proven), $r\left(\mathcal{Q}_{1, n}\right)$ and $\mathcal{E}$ (seen as a class of constant functions with constant envelope $c$ ) are separable, (A.37) implies that $\mathcal{F}_{n}$ is separable. Moreover, (A.37) also implies

$$
J_{c}\left(1, \mathcal{F}_{n}\right) \lesssim J_{c}\left(1, \mathcal{Q}_{1, n}\right)+J_{c}\left(1, r\left(\mathcal{Q}_{1, n}\right)\right)+J_{c}(1, \mathcal{E})
$$

(see the argument following (A.20)) which by $\mathbf{A} 4$ yields in turn that $J_{c}\left(1, \mathcal{F}_{n}\right)=o(\sqrt{n})$. Thus, $\left(\mathcal{F}_{n}\right)^{2}$ is separable, $J_{c}\left(1,\left(\mathcal{F}_{n}\right)^{2}\right)=o(\sqrt{n})$, Lemma B.3 applies and teaches us that $E\left(\left\|P_{n}-P_{Q_{0}, \mathbf{g}_{n}}\right\|_{\left(\mathcal{F}_{n}\right)^{2}}\right)=o(1)$, and finally Markov's inequality implies $\left\|P_{n}-P_{Q_{0}, \mathbf{g}_{n}}\right\|_{\left(\mathcal{F}_{n}\right)^{2}}=o_{P}(1)$. This completes the study of the first term in the RHS of (A.36). 
- To rely more easily on all the results obtained so far, we first note that

$$
\begin{aligned}
\left|P_{Q_{0}, g_{0}}\left(f_{n}^{2}-f_{0}^{2}\right)\right| & \leq\left|P_{Q_{0}, g_{0}}\left(f_{n}^{2}-f_{0, n}^{2}\right)\right|+\left|P_{Q_{0}, g_{0}}\left(f_{0, n}^{2}-f_{0}^{2}\right)\right| \\
& \leq P_{Q_{0}, g_{0}}\left|f_{n}^{2}-f_{0, n}^{2}\right|+P_{Q_{0}, g_{0}}\left|f_{0, n}^{2}-f_{0}^{2}\right| \\
& \lesssim P_{Q_{0}, g_{0}}\left|f_{n}-f_{0, n}\right|+P_{Q_{0}, g_{0}}\left|f_{0, n}-f_{0}\right| \\
& \leq\left\|f_{n}-f_{0, n}\right\|_{2, P_{Q_{0}, g^{\text {ref }}}}+\left\|f_{0, n}-f_{0}\right\|_{2, P_{Q_{0}, g^{\text {ref }}}}
\end{aligned}
$$

where the last upper-bound follows from the Cauchy-Schwarz inequality and the fact that $g_{0}$ and $g^{\text {ref }}$ are bounded away from 0 and 1 . Now,

$$
\begin{aligned}
\left\|f_{n}-f_{0, n}\right\|_{2, P_{Q_{0}, g^{\mathrm{ref}}} \leq \|} \leq\left(Q_{Y, \zeta_{n}, r_{n}}^{*}-Q_{Y, \zeta_{0}, r_{n}}^{*}\right) \circ r_{n} \|_{2, P_{Q_{0}, g^{\mathrm{ref}}}} \\
+\left\|d_{Y, n}^{*}-d_{Y, \zeta_{0}, r_{n}}^{*}\right\|_{2, P_{Q_{0}, g} \mathrm{ref}}
\end{aligned}
$$

and we already proved that $\left\|\left(Q_{Y, \zeta_{n}, r_{n}}^{*}-Q_{Y, \zeta_{0}, r_{n}}^{*}\right) \circ r_{n}\right\|_{2, P_{Q_{0}, g^{\mathrm{ref}}}}=$ $o_{P}(1)$ (see step two of the proof of Proposition A.1) and $\| d_{Y, n}^{*}-$ $d_{Y, \zeta_{0}, r_{n}}^{*}\left\|_{2, P_{Q_{0}, g^{\text {ref }}}}=\right\| \Delta d_{Y, \beta_{n}}\left(\epsilon_{n}\right) \|_{2, P_{Q_{0}, g^{\text {ref }}}}=o_{P}(1)$ (see step two of proof of Proposition A.2). Therefore, $\left\|f_{n}-f_{0, n}\right\|_{2, P_{Q_{0}, g^{\mathrm{ref}}}}=o_{P}(1)$ and it suffices to show that $\left\|f_{0, n}-f_{0}\right\|_{2, P_{Q_{0}, g} \text { ref }}=o_{P}(1)$ too to obtain the desired convergence $P_{Q_{0}, g_{0}}\left(f_{n}^{2}-f_{0}^{2}\right)=o_{P}(1)$.

As previously, we first note that

$$
\begin{aligned}
\left\|f_{0, n}-f_{0}\right\|_{2, P_{Q_{0}, g^{\mathrm{ref}}} \leq \| Q_{Y, \zeta_{0}, r_{n}}^{*} \circ r_{n}} & -Q_{Y, \zeta_{0}, r_{0}}^{*} \circ r_{0} \|_{2, P_{Q_{0}, g^{\mathrm{ref}}}} \\
& +\left\|d_{Y, \zeta_{0}, r_{n}}^{*}-d_{Y, \zeta_{0}, r_{0}}^{*}\right\|_{2, P_{Q_{0}, g^{\mathrm{ref}}}} .
\end{aligned}
$$

By (A.20) and (A.32) in step two of the proof of Proposition A.2, it holds that

$$
\begin{aligned}
\left\|Q_{Y, \zeta_{0}, r_{n}}^{*} \circ r_{n}-Q_{Y, \zeta_{0}, r_{0}}^{*} \circ r_{0}\right\|_{2, P_{Q_{0}, g^{\mathrm{ref}}}} \lesssim\left\|\epsilon_{0}\left(r_{n}\right)-\epsilon_{0}\left(r_{0}\right)\right\|_{2, P_{Q_{0}, g^{\mathrm{ref}}}} \\
+\left\|r_{n}-r_{0}\right\|_{2, P_{Q_{0}, g^{\mathrm{ref}}}} \\
\lesssim\left\|r_{n}-r_{0}\right\|_{2, P_{Q_{0}, g^{\mathrm{ref}}}}
\end{aligned}
$$

with $\left\|r_{n}-r_{0}\right\|_{2, P_{Q_{0}, g} \text { ref }}=o_{P}(1)$ by Proposition 4.1, whose assumptions are met. Once again, we control the last remaining term in the same spirit as in step two of the proof of Proposition A.2: from the upperbound

$$
\left|\left(d_{Y, \zeta_{0}, r_{n}}^{*}-d_{Y, \zeta_{0}, r_{0}}^{*}\right)(O, Z)\right|
$$




$$
\begin{gathered}
\left.\lesssim \mid \mathbf{1}\left\{A=r_{n}(W)\right\}\left(Q_{Y, \zeta_{0}, r_{n}}^{*}-Q_{Y, \zeta_{0}, r_{0}}^{*}\right)\right)(A, W) \mid \\
\quad+\left|\mathbf{1}\left\{A=r_{n}(W)\right\}-\mathbf{1}\left\{A=r_{0}(W)\right\}\right| \\
\lesssim\left|\epsilon_{0}\left(r_{n}\right)-\epsilon_{0}\left(r_{0}\right)\right|+\left|r_{n}(W)-r_{0}(W)\right|
\end{gathered}
$$

we deduce that

$$
\left\|d_{Y, \zeta_{0}, r_{n}}^{*}-d_{Y, \zeta_{0}, r_{0}}^{*}\right\|_{2, P_{Q_{0}, g^{\mathrm{ref}}}} \lesssim\left\|r_{n}-r_{0}\right\|_{2, P_{Q_{0}, g^{\mathrm{ref}}}}=o_{P}(1)
$$

In summary, $\left\|f_{0, n}-f_{0}\right\|_{2, P_{Q_{0}, g^{\mathrm{ref}}}}=o_{P}(1)$, and this completes the study of the second term in the RHS of (A.36).

By combining the results of the above two-step study of the RHS sum in (A.36) and (A.36) itself we finally get the stated convergence $\Sigma_{n}=\Sigma_{0}+$ $o_{P}(1)$, thus completing step two of the current proof.

Step three: deriving (4.15) from (A.21). The asymptotic linear expansion (A.21) rewrites as

$$
\begin{aligned}
\psi_{n}^{*}-\psi_{r_{n}, 0} & =\left(P_{n}-P_{Q_{0}, \mathbf{g}_{n}}\right) f_{0, n}+o_{P}(1 / \sqrt{n}) \\
& =\left(P_{n}-P_{Q_{0}, \mathbf{g}_{n}}\right) f_{0}+\left(P_{n}-P_{Q_{0}, \mathbf{g}_{n}}\right)\left(f_{0, n}-f_{0}\right)+o_{P}(1 / \sqrt{n}),
\end{aligned}
$$

hence (4.15) follows from the convergence

$$
\left(P_{n}-P_{Q_{0}, \mathbf{g}_{n}}\right)\left(f_{0, n}-f_{0}\right)=o_{P}(1 / \sqrt{n}),
$$

which is a consequence of Lemma B.4.

For each $n \geq 1$, introduce the class

$$
\mathcal{F}_{n}^{\prime} \equiv\left\{Q_{Y, \zeta_{0}, \rho}^{*} \circ \rho+d_{Y, \zeta_{0}, \rho}^{*}-f_{0}: \rho \in r\left(\mathcal{Q}_{1, n}\right)\right\} .
$$

In particular, $f_{0, n}-f_{0} \in \mathcal{F}_{n}^{\prime}$ (take $\rho=r_{n}$ ), and we have already proven in the previous step of the current proof that $\left\|f_{0, n}-f_{0}\right\|_{2, P_{Q_{0}, g^{\text {ref }}}}=o_{P}(1)$. The class $\mathcal{F}_{n}^{\prime}$ is uniformly bounded, so there exists a constant $c^{\prime} \geq 1$ which can serve as an envelope function to both $\mathcal{F}_{n}^{\prime}$ and $r\left(\mathcal{Q}_{1, n}\right)$. Obviously, the resulting (constant) sequence of (constant) envelope functions satisfies condition (a) in Lemma B.4. Set arbitrarily $\rho_{1}, \rho_{2} \in r\left(\mathcal{Q}_{1, n}\right)$. We have

$$
\begin{aligned}
& \left|\left(Q_{Y, \zeta_{0}, \rho_{1}}^{*} \circ \rho_{1}+d_{Y, \zeta_{0}, \rho_{1}}^{*}-f_{0}\right)-\left(Q_{Y, \zeta_{0}, \rho_{1}}^{*} \circ \rho_{2}+d_{Y, \zeta_{0}, \rho_{2}}^{*}-f_{0}\right)\right| \\
& \quad \leq\left|Q_{Y, \zeta_{0}, \rho_{1}}^{*} \circ \rho_{1}-Q_{Y, \zeta_{0}, \rho_{1}}^{*} \circ \rho_{2}\right|+\left|d_{Y, \zeta_{0}, \rho_{1}}^{*}-d_{Y, \zeta_{0}, \rho_{2}}^{*}\right|
\end{aligned}
$$

By (A.20), (A.32) in step two of the proof of Proposition A.2 and (A.39) with $\left(\rho_{1}, \rho_{2}\right)$ substituted for $\left(r_{n}, r_{0}\right)$, this inequality yields 


$$
\begin{aligned}
&\left|\left(Q_{Y, \zeta_{0}, \rho_{1}}^{*} \circ \rho_{1}+d_{Y, \zeta_{0}, \rho_{1}}^{*}-f_{0}\right)-\left(Q_{Y, \zeta_{0}, \rho_{1}}^{*} \circ \rho_{2}+d_{Y, \zeta_{0}, \rho_{2}}^{*}-f_{0}\right)\right| \\
& \lesssim\left|\epsilon_{0}\left(\rho_{1}\right)-\epsilon_{0}\left(\rho_{2}\right)\right|+\left|\rho_{1}-\rho_{2}\right| \lesssim\left\|\rho_{1}-\rho_{2}\right\|_{2, P_{Q_{0}, g^{\text {ref }}}}+\left|\rho_{1}-\rho_{2}\right|
\end{aligned}
$$

Consequently, $\mathcal{F}_{n}^{\prime}$ is separable because $r\left(\mathcal{Q}_{1, n}\right)$ is separable. Moreover, since the definition of the uniform entropy integral involve a supremum over probability measures, the above pointwise inequality entails that, for each $\delta>0$, $J_{c^{\prime}}\left(\delta, \mathcal{F}_{n}^{\prime}\right) \lesssim J_{c^{\prime}}\left(\delta, r\left(\mathcal{Q}_{1, n}\right)\right)$, so that condition (b) in Lemma B.4 is met by A4*. Applying Lemma B.4 then gives $\left(P_{n}-P_{Q_{0}, \mathbf{g}_{n}}\right)\left(f_{0, n}-f_{0}\right)=o_{P}(1 / \sqrt{n})$, hence the validity of (4.15).

Step four: deducing the limiting normal distribution from (4.15). We first argue that (4.15) implies the converges in law to the standard normal distribution of $\sqrt{n / \Sigma_{0}}\left(\psi_{n}^{*}-\psi_{0}\right)$. This is a consequence of [5, Theorem 3.3.7] because (i) $S_{n} / E\left(S_{n}\right)-1=o_{P}(1)$, and (ii) for each $\alpha>0, E\left(P_{n} f_{0}^{2} \mathbf{1}\left\{f_{0}^{2} \geq\right.\right.$ $\left.\left.\alpha^{2} n E\left(S_{n}\right)\right\}\right)=o\left(E\left(S_{n}\right)\right)$ trivially holds since $f_{0}$ is bounded and $E\left(S_{n}\right)=$ $\Sigma_{0}+o(1)$ with $\Sigma_{0}>0$. Then Slutsky's lemma and $\Sigma_{n}=\Sigma_{0}+o_{P}(1)$ yield the convergence in law of $\sqrt{n / \Sigma_{n}}\left(\psi_{n}^{*}-\psi_{0}\right)$ to the same limiting distribution. This completes the proof.

Lemma A.2. The convergence $\left\|g_{n}-g_{0}\right\|_{2, P_{Q_{0}, g^{\mathrm{ref}}}}=o_{P}(1)$ implies that $\left\|n^{-1} \sum_{i=1}^{n}\left(g_{i}-g_{0}\right)\right\|_{2, P_{Q_{0}, g} \text { ref }}$ converges to 0 both in probability and in $L^{1}$.

Proof of Lemma A.2. Since $\mathcal{G}$ is uniformly bounded, the convergence $\left\|g_{n}-g_{0}\right\|_{2, P_{Q_{0}, g^{\mathrm{ref}}}}=o_{P}(1)$ implies $E\left(\left\|g_{n}-g_{0}\right\|_{2, P_{Q_{0}, g^{\mathrm{ref}}}}\right)=o(1)$. Now, by convexity then Cesaro's lemma,

$$
E\left(\left\|\frac{1}{n} \sum_{i=1}^{n}\left(g_{i}-g_{0}\right)\right\|_{2, P_{Q_{0}, g^{\mathrm{ref}}}}\right) \leq \frac{1}{n} \sum_{i=1}^{n} E\left(\left\|\left(g_{i}-g_{0}\right)\right\|_{2, P_{Q_{0}, g^{\mathrm{ref}}}}\right)=o(1) .
$$

This convergence in $L^{1}$ implies the convergence in probability because $\mathcal{G}$ is uniformly bounded.

\section{A.3. Proofs of Propositions 4.2, 5.2, 5.3 and 5.4.}

Proof of Proposition 4.2. Set a probability measure $\tilde{\mu}$ on the measured space $\mathcal{A} \times \mathcal{W}$. Denote $\bar{\mu}$ the marginal probability measure induced by $\tilde{\mu}$ on $\mathcal{W}$. Let $\left\{\delta_{n}\right\}_{n \geq 1}$ be a sequence of positive numbers such that $\delta_{n}=o(1)$ and set $\varepsilon>0$.

Since $r\left(\mathcal{Q}_{1, n}\right)$ is a subset of a fixed VC-class of functions taking values in $[0,1]$, there exists a constant $c>0$ such that, for all $0<\varepsilon<1$,

$$
\log \sup _{\mu} N\left(\varepsilon\|1\|_{2, \mu}, r\left(\mathcal{Q}_{1, n}\right),\|\cdot\|_{2, \mu}\right) \lesssim \log \left(\varepsilon^{-1}\right)+c
$$


[7, Theorem 2.6.7], where 1 serves as a fixed (and constant) envelope function for $r\left(\mathcal{Q}_{1, n}\right)$ and the supremum is taken over all probability measures $\mu$ on $\mathcal{W}$. It follows easily that $J_{1}\left(\delta_{n}, r\left(\mathcal{Q}_{1, n}\right)\right) \lesssim \int_{0}^{\delta_{n}} \sqrt{\log \left(\varepsilon^{-1}\right)+c} d \varepsilon=o(1)$. In particular, the choice $\delta_{n}=1 / \sqrt{n}$ yields $J_{1}\left(1, r\left(\mathcal{Q}_{1, n}\right)\right)=o(\sqrt{n})$.

We now turn to $\mathcal{Q}_{1, n}$. Let $\left\{f_{j}^{-}: 1 \leq j \leq N\left(\varepsilon, \mathcal{F}^{-},\|\cdot\|_{2, \bar{\mu}}\right)\right\}$ and $\left\{f_{j}^{+}\right.$: $\left.1 \leq j \leq N\left(\varepsilon, \mathcal{F}^{+},\|\cdot\|_{2, \bar{\mu}}\right)\right\}$ be two collections of functions from $\mathcal{W}$ to $\mathbb{R}$ such that the unions of the $L^{2}(\bar{\mu})$-balls of radius $\varepsilon$ centered at $f_{j}^{-}$or $f_{j}^{+}$cover $\mathcal{F}^{-}$ or $\mathcal{F}^{+}$, respectively. Choose arbitrarily $Q_{Y, \beta} \in \mathcal{Q}_{1, n}$, with $\beta \equiv\left(f^{-}, f^{+}\right) \in$ $B_{n}$. We may assume without loss of generality that $\left\|f^{-}-f_{1}^{-}\right\|_{2, \bar{\mu}} \leq \varepsilon$ and $\left\|f^{+}-f_{1}^{+}\right\|_{2, \bar{\mu}} \leq \varepsilon$. Introduce $\beta_{1} \equiv\left(f_{1}^{-}, f_{1}^{+}\right)$and $Q_{Y, \beta_{1}}$ defined as in (4.7) with $\beta_{1}$ substituted for $\beta$ (the fact that $\beta_{1}$ may fall outside $B_{n}$ is not a concern). Now, observe that

$$
\left|Q_{Y, \beta}-Q_{Y, \beta_{1}}\right|^{2} \leq\left(\left|f^{-}-f_{1}^{-}\right|+\left|f^{+}-f_{1}^{+}\right|\right)^{2} \leq 2\left(\left|f^{-}-f_{1}^{-}\right|^{2}+\left|f^{+}-f_{1}^{+}\right|^{2}\right)
$$

hence

$$
\left\|Q_{Y, \beta}-Q_{Y, \beta_{1}}\right\|_{2, \tilde{\mu}} \leq \sqrt{2}\left(\left\|f^{-}-f_{1}^{-}\right\|_{2, \bar{\mu}}+\left\|f^{+}-f_{1}^{+}\right\|_{2, \bar{\mu}}\right) \leq 2 \sqrt{2} \varepsilon .
$$

This entails that

$$
N\left(\varepsilon, \mathcal{Q}_{1, n},\|\cdot\|_{2, \tilde{\mu}}\right) \leq N\left(\varepsilon / 2 \sqrt{2}, \mathcal{F}^{-},\|\cdot\|_{2, \tilde{\mu}}\right) \times N\left(\varepsilon / 2 \sqrt{2}, \mathcal{F}^{+},\|\cdot\|_{2, \tilde{\mu}}\right) .
$$

Since $\|1\|_{2, \bar{\mu}}=1,\|2\|_{2, \bar{\mu}}=2$ and because $\|1\|_{2, \tilde{\mu}}=1$ where 1 serves as a (constant) envelope function to $\mathcal{Q}_{1, n},(4.5),(4.6)$ and the previous bound imply the existence of $\alpha \in[0,1)$ (independent of $\tilde{\mu}$ ) such that

$$
\sqrt{\log N\left(\varepsilon\|1\|_{2, \tilde{\mu}}, \mathcal{Q}_{1, n},\|\cdot\|_{2, \tilde{\mu}}\right)} \lesssim\left(\frac{1}{\varepsilon}\right)^{\alpha} .
$$

Taking the supremum over all probability measures $\tilde{\mu}$ on the measured space $\mathcal{A} \times \mathcal{W}$ and integrating wrt $\varepsilon$ then yield $J_{1}\left(\delta_{n}, \mathcal{Q}_{1, n}\right)=o(1)$. In particular, the choice $\delta_{n}=1 / \sqrt{n}$ gives $J_{1}\left(1, \mathcal{Q}_{1, n}\right)=o(\sqrt{n})$.

We now turn to $L^{\mathrm{ls}}\left(\mathcal{Q}_{1, n}\right)$, which admits 1 as a (constant) envelope function. Simply observe that

$$
\begin{aligned}
\mid L\left(Q_{Y, \beta}\right)(O)- & L\left(Q_{Y, \beta_{1}}\right)(O) \mid \\
& =\left|\left(Y-Q_{Y, \beta}(A, W)\right)^{2}-\left(Y-Q_{Y, \beta_{1}}(A, W)\right)^{2}\right| \\
& =\left|2 Y-Q_{Y, \beta}(O)-Q_{Y, \beta_{1}}(O)\right| \times\left|Q_{Y, \beta}(O)-Q_{Y, \beta_{1}}(O)\right| \\
& \lesssim\left|Q_{Y, \beta}(O)-Q_{Y, \beta_{1}}(O)\right|,
\end{aligned}
$$

which entails $J_{1}\left(1, L^{\mathrm{ls}}\left(\mathcal{Q}_{1, n}\right)\right)=O\left(J_{1}\left(1, \mathcal{Q}_{1, n}\right)\right)=o(1)$. This completes the proof. 
Proof of Proposition 5.2. Arbitrarily set $t>0$. By the LHS equality in (A.8), shown while proving Lemma 3.1, we first get

$$
\begin{aligned}
0 \leq \psi_{0}-\psi_{r_{n}, 0} \leq & E_{Q_{0}}\left(\left|q_{Y, 0}(W)\right| \times\left|r_{n}(W)-r_{0}(W)\right|\right) \\
= & E_{Q_{0}}\left(\left|q_{Y, 0}(W)\right| \times \mathbf{1}\left\{r_{n}(W) \neq r_{0}(W)\right\}\right) \\
= & E_{Q_{0}}\left(\left|q_{Y, 0}(W)\right| \times \mathbf{1}\left\{r_{n}(W) \neq r_{0}(W)\right\}\right. \\
& \left.\quad \times\left(\mathbf{1}\left\{\left|q_{Y, 0}(W)\right| \geq t\right\}+\mathbf{1}\left\{\left|q_{Y, 0}(W)\right|<t\right\}\right)\right) .
\end{aligned}
$$

Recall that $r_{n}(W) \neq r_{0}(W)$ is equivalent to $q_{Y, \beta_{n}} q_{Y, \beta_{0}}(W)<0$ and therefore implies $\left|\left(q_{Y, \beta_{n}}-q_{Y, 0}\right)(W)\right| \geq\left|q_{Y, 0}(W)\right|$. Thus, the above inequality entails that $0 \leq \psi_{0}-\psi_{r_{n}, 0}$ is smaller than

$$
\begin{aligned}
& E_{Q_{0}}\left(\left|\left(q_{Y, \beta_{n}}-q_{Y, 0}\right)(W)\right| \times \mathbf{1}\left\{\left|\left(q_{Y, \beta_{n}}-q_{Y, 0}\right)(W)\right| \geq\left|q_{Y, 0}(W)\right| \geq t\right\}\right) \\
+ & E_{Q_{0}}\left(\left|q_{Y, 0}(W)\right|^{1 / 3} \times\left|\left(q_{Y, \beta_{n}}-q_{Y, 0}\right)(W)\right|^{2 / 3} \times \mathbf{1}\left\{\left|q_{Y, 0}(W)\right|<t\right\}\right)
\end{aligned}
$$

First, we note that the first term in (A.41) is bounded by

$$
\begin{aligned}
& E_{Q_{0}}\left(\left|\left(q_{Y, \beta_{n}}-q_{Y, 0}\right)(W)\right| \times \mathbf{1}\left\{\left|\left(q_{Y, \beta_{n}}-q_{Y, 0}\right)(W)\right| \geq\left|q_{Y, 0}(W)\right| \geq t\right\}\right) \\
& \quad \leq E_{Q_{0}}\left(\frac{\left|q_{Y, 0}(W)\right|}{t} \times \frac{\left(q_{Y, \beta_{n}}-q_{Y, 0}\right)^{2}(W)}{t}\right)=t^{-2}\left\|q_{Y, \beta_{n}}-q_{Y, 0}\right\|_{2}^{2} .
\end{aligned}
$$

Second, Hölder's inequality and A5* yield that second term in (A.41) is bounded by

$$
\left\|q_{Y, \beta_{n}}-q_{Y, 0}\right\|_{2}^{2 / 3} \times P_{Q_{0}}\left(0<\left|q_{Y, 0}(W)\right| \leq t\right)^{2 / 3} \lesssim t^{2 \gamma_{2} / 3}\left\|q_{Y, \beta_{n}}-q_{Y, 0}\right\|_{2}^{2 / 3} .
$$

In summary, we have proven that

$$
0 \leq \psi_{0}-\psi_{r_{n}, 0} \lesssim t^{-2}\left\|q_{Y, \beta_{n}}-q_{Y, 0}\right\|_{2}^{2}+t^{2 \gamma_{2} / 3}\left\|q_{Y, \beta_{n}}-q_{Y, 0}\right\|_{2}^{2 / 3}
$$

Optimizing in $t$ finally yields (5.1). In conclusion, $\left\|q_{Y, \beta_{n}}-q_{Y, 0}\right\|_{2}=o_{P}\left(1 / n^{\gamma_{3}}\right)$ does imply $\psi_{0}-\psi_{r_{n}, 0}=o_{P}(1 / \sqrt{n})$ because $2\left(1+\gamma_{2}\right) /\left(3+\gamma_{2}\right) \times \gamma_{3}=1 / 2$.

The claim on the confidence interval readily follows from Proposition 5.1 and the property $\psi_{0}-\psi_{r_{n}, 0}=o_{P}(1 / \sqrt{n})$. This completes the proof.

Proof of Proposition 5.3. Since $\psi_{n}^{*}$ and $n^{-1} \sum_{i=1}^{n} Y_{i}$ are known quantities, we focus on

$$
\sqrt{n} \Omega_{n}^{\varepsilon} \equiv \sqrt{n}\left(\psi_{n}^{*}+\mathcal{E}_{n}-\frac{1}{n} \sum_{i=1}^{n} Y_{i}\right)=\sqrt{n}\left(\psi_{n}^{*}-P_{n} Q_{Y, 0} \circ r_{n}\right) .
$$


By definition of $\psi_{r_{n}, 0}(4.4)$ and (4.15), it holds that

$$
\begin{aligned}
\sqrt{n} \Omega_{n}^{\mathcal{E}}= & \sqrt{n}\left(\psi_{n}^{*}-\psi_{r_{n}, 0}\right)-\sqrt{n}\left(P_{n}-P_{Q_{0}, \mathbf{g}_{n}}\right) Q_{Y, 0} \circ r_{n} \\
= & \sqrt{n}\left(P_{n}-P_{Q_{0}, \mathbf{g}_{n}}\right)\left(d_{Y, 0}^{*}+d_{W, 0}^{*}-Q_{Y, 0} \circ r_{0}\right) \\
& \quad+\sqrt{n}\left(P_{n}-P_{Q_{0}, \mathbf{g}_{n}}\right) Q_{Y, 0} \circ\left(r_{n}-r_{0}\right)+o_{P}(1) .
\end{aligned}
$$

Arguments similar to those developed in Section A.2 to prove Corollary A.1 successively yield $\Sigma_{n}^{\mathcal{E}}=\Sigma_{0}^{\mathcal{E}}+o_{P}(1), \sqrt{n}\left(P_{n}-P_{Q_{0}, \mathbf{g}_{n}}\right) Q_{Y, 0} \circ\left(r_{n}-r_{0}\right)=o_{P}(1)$,

$$
\sqrt{n} \Omega_{n}^{\mathcal{E}}=\sqrt{n}\left(P_{n}-P_{Q_{0}, \mathbf{g}_{n}}\right)\left(d_{Y, 0}^{*}+Q_{W, \zeta_{0}, r_{0}}^{*}-Q_{Y, 0} \circ r_{0}\right)+o_{P}(1)
$$

and the convergence in distribution of $\sqrt{n / \Sigma_{n}^{\varepsilon}} \Omega_{n}^{\mathcal{E}}$ to the standard normal distribution. This justifies the validity of the proposed asymptotic confidence interval.

Proof of Proposition 5.4. This is a three-step proof.

Step one: preliminary. Let us assume for the time being that we also observe the variables $U_{1}, \ldots, U_{n}$ in addition to $O_{1}, \ldots, O_{n}$. The resulting counterpart to $\mathbf{O}_{n}$ is denoted $\mathbb{O}_{n} \equiv\left(\left(O_{1}, U_{1}\right), \ldots,\left(O_{n}, U_{n}\right)\right)$ with convention $\mathbb{O}_{0} \equiv \emptyset$. Likewise, the resulting counterpart to the empirical measure $P_{n}$ is $\mathbb{P}_{n}$. Since the sequence $\left\{U_{n}\right\}_{n \geq 1}$ consists of i.i.d. variables independent from $\left\{O_{n}\right\}_{n \geq 1}$, a distribution $P_{Q, g} \in \mathcal{M}$ for $(O, Z)$ yields univocally a distribution $\mathbb{P}_{Q, g}$ for $(O, Z, U)$. For a measurable function $f: \mathcal{O} \times[0,1] \times \mathcal{U} \rightarrow \mathbb{R}^{d}$, we denote $\mathbb{P}_{n} f \equiv n^{-1} \sum_{i=1}^{n} f\left(O_{i}, Z_{i}, U_{i}\right)$ and $\mathbb{P}_{Q, g} f \equiv E_{\mathbb{P}_{Q, g}}(f(O, Z, U))$.

Neglecting this new source of information, we carry out the exact same statistical procedure as developed and studied in Sections 2, 3, 4, 5.1 and 5.2. If we write

$$
\begin{aligned}
\mathbb{P}_{Q_{0}, g_{i}} f & \equiv E_{\mathbb{P}_{Q_{0}, g_{i}}}\left[f\left(O_{i}, Z_{i}, U_{i}\right) \mid \mathbb{O}_{i-1}\right] \\
\mathbb{P}_{Q_{0}, \mathbf{g}_{n}} f & \equiv \frac{1}{n} \sum_{i=1}^{n} \mathbb{P}_{Q_{0}, g_{i}} f
\end{aligned}
$$

for the counterparts to $P_{Q_{0}, g_{i}} f$ and $P_{Q_{0}, \mathbf{g}_{n}} f$ (each $\left.i=1, \ldots, n\right)$, then (A.42) reads

$$
\sqrt{n} \Omega_{n}^{\mathcal{E}}=\sqrt{n}\left(\psi_{n}^{*}-\mathbb{P}_{n} Q_{Y, 0} \circ r_{n}\right)
$$

and (A.43) still holds and reads

$$
\sqrt{n} \Omega_{n}^{\mathcal{E}}=\sqrt{n}\left(\mathbb{P}_{n}-\mathbb{P}_{Q_{0}, \mathbf{g}_{n}}\right)\left(d_{Y, 0}^{*}+Q_{W, \zeta_{0}, r_{0}}^{*}-Q_{Y, 0} \circ r_{0}\right)+o_{P}(1) .
$$


Step two: inferring in the causal world. For $\rho=r_{0}$ and $\rho=r_{n}$, we set $\mathbb{Q}_{Y, 0} \circ \rho(W, U)=\mathbb{Q}_{Y, 0}(\rho(W), W, U)$. Since $\psi_{n}^{*}$ and $n^{-1} \sum_{i=1}^{n} Y_{i}$ are known quantities, we focus on

$$
\sqrt{n} \Omega_{n}^{\mathcal{e}} \equiv \sqrt{n}\left(\psi_{n}^{*}+\mathcal{C}_{n}-\frac{1}{n} \sum_{i=1}^{n} Y_{i}\right)=\sqrt{n}\left(\psi_{n}^{*}-\mathbb{P}_{n} \mathbb{Q}_{Y, 0} \circ r_{n}\right) .
$$

By (A.44), (A.45), and because (5.6) implies $\mathbb{P}_{Q_{0}, \mathbf{g}_{n}}\left(\mathbb{Q}_{Y, 0}-Q_{Y, 0}\right) \circ r_{n}=$ $\mathbb{P}_{Q_{0}, g_{0}}\left(\mathbb{Q}_{Y, 0}-Q_{Y, 0}\right) \circ r_{n}=0$, it holds that

$$
\begin{aligned}
\sqrt{n} \Omega_{n}^{\mathcal{C}} \\
=\sqrt{n} \Omega_{n}^{\mathcal{E}}-\sqrt{n} \mathbb{P}_{n}\left(\mathbb{Q}_{Y, 0} \circ r_{n}-Q_{Y, 0} \circ r_{n}\right) \\
=\sqrt{n}\left(\mathbb{P}_{n}-\mathbb{P}_{Q_{0}, \mathbf{g}_{n}}\right)\left(d_{Y, 0}^{*}+Q_{W, \zeta_{0}, r_{0}}^{*}-Q_{Y, 0} \circ r_{0}\right) \\
\quad-\sqrt{n}\left(\mathbb{P}_{n}-\mathbb{P}_{Q_{0}, \mathbf{g}_{n}}\right)\left(\mathbb{Q}_{Y, 0}-Q_{Y, 0}\right) \circ r_{n}+o_{P}(1) \\
=\sqrt{n}\left(\mathbb{P}_{n}-\mathbb{P}_{Q_{0}, \mathbf{g}_{n}}\right)\left(d_{Y, 0}^{*}+Q_{W, \zeta_{0}, r_{0}}^{*}-Q_{Y, 0} \circ r_{0}\right) \\
\quad-\sqrt{n}\left(\mathbb{P}_{n}-\mathbb{P}_{Q_{0}, \mathbf{g}_{n}}\right)\left(\mathbb{Q}_{Y, 0}-Q_{Y, 0}\right) \circ r_{0} \\
\quad-\sqrt{n}\left(\mathbb{P}_{n}-\mathbb{P}_{Q_{0}, \mathbf{g}_{n}}\right)\left(\left(\mathbb{Q}_{Y, 0}-Q_{Y, 0}\right) \circ r_{n}-\left(\mathbb{Q}_{Y, 0}-Q_{Y, 0}\right) \circ r_{0}\right)+o_{P}(1) .
\end{aligned}
$$

Define $f_{0} \equiv d_{Y, 0}^{*}+Q_{W, \zeta_{0}, r_{0}}^{*}-\left(Q_{Y, 0} \circ r_{0}-\psi_{0}\right), \chi_{0} \equiv\left(\mathbb{Q}_{Y, 0}-Q_{Y, 0}\right) \circ r_{0}$, and $\Sigma_{0}^{\mathcal{C}} \equiv \mathbb{P}_{Q_{0}, g_{0}}\left(f_{0}-\chi_{0}\right)^{2}$. Arguments similar to those developed in Section A.2 to prove Corollary A.1 successively yield

$$
\begin{gathered}
\sqrt{n}\left(\mathbb{P}_{n}-\mathbb{P}_{Q_{0}, \mathbf{g}_{n}}\right)\left(\left(\mathbb{Q}_{Y, 0}-Q_{Y, 0}\right) \circ r_{n}-\chi_{0}\right)=o_{P}(1), \\
\sqrt{n} \Omega_{n}^{\mathcal{C}}=\sqrt{n}\left(\mathbb{P}_{n}-\mathbb{P}_{Q_{0}, \mathbf{g}_{n}}\right)\left(f_{0}-\chi_{0}\right)+o_{P}(1)
\end{gathered}
$$

and the convergence in distribution of $\sqrt{n / \Sigma_{0}^{\mathcal{e}}} \Omega_{n}^{\mathcal{e}}$ to the standard normal distribution.

Step three: inferring in the real world. At this stage, there is still one issue to solve: it is not possible to infer $\Sigma_{0}^{e}$ because, contrary to $f_{0}$ which is a function of $O, \chi_{0}$ is a function of $(O, U)$ and we actually do not observe $U_{1}, \ldots, U_{n}$. Fortunately, it holds that

$$
\Sigma_{0}^{\mathcal{C}}=P_{Q_{0}, g_{0}} f_{0}^{2}-\mathbb{P}_{Q_{0}, g_{0}} \chi_{0}^{2}=\Sigma_{0}^{\mathcal{E}}-\mathbb{P}_{Q_{0}, g_{0}} \chi_{0}^{2} \leq \Sigma_{0}^{\mathcal{E}},
$$

the inequality justifying our claim on the proposed asymptotic confidence interval.

It only remains to prove the LHS equality in (A.46), which is equivalent to $\mathbb{P}_{Q_{0}, g_{0}} f_{0} \chi_{0}=\mathbb{P}_{Q_{0}, g_{0}} \chi_{0}^{2}$. First, we note that

$$
\mathbb{P}_{Q_{0}, g_{0}} f_{0} \chi_{0}=\mathbb{P}_{Q_{0}, g_{0}}\left(Q_{W, \zeta_{0}, r_{0}}^{*}-\left(Q_{Y, 0} \circ r_{0}-\psi_{0}\right)\right) \chi_{0}+\mathbb{P}_{Q_{0}, g_{0}} d_{Y, 0}^{*} \chi_{0} .
$$


By the tower rule and (5.6), the first RHS term in this sum equals

$$
\begin{aligned}
& E_{\mathbb{P}_{Q_{0}, g_{0}}}\left[\left(Q_{W, \zeta_{0}, r_{0}}^{*}(W)-\left(Q_{Y, 0} \circ r_{0}(W)-\psi_{0}\right)\right)\right. \\
&\left.\quad \times E_{\mathbb{P}_{Q_{0}, g_{0}}}\left(\mathbb{Q}_{Y, 0}\left(r_{0}(W), W, U\right)-Q_{Y, 0}\left(r_{0}(W), W\right) \mid W\right)\right]=0 .
\end{aligned}
$$

Thus, proving the LHS equality in (A.46) boils down to showing that the second term equals $\mathbb{P}_{Q_{0}, g_{0}} \chi_{0}^{2}$. By definitions of $d_{Y, 0}^{*}$ (4.10) and of $Y$ in the causal model, the tower rule and (5.6) imply that the second term equals

$$
\begin{aligned}
& E_{\mathbb{P}_{Q_{0}, g_{0}}}\left[\frac{\mathbf{1}\left\{A=r_{0}(W)\right\}}{Z}\left(\mathbb{Q}_{Y, 0}\left(r_{0}(W), W, U\right)-Q_{Y, 0}\left(r_{0}(W), W\right)\right)\right. \\
& \left.\times E_{\mathbb{P}_{Q_{0}, g_{0}}}\left(Y-Q_{Y, 0}^{*}\left(r_{0}(W), W\right) \mid A, W, U\right)\right] \\
& =E_{\mathbb{P}_{Q_{0}, g_{0}}}\left[\frac{1\left\{A=r_{0}(W)\right\}}{Z}\left(\mathbb{Q}_{Y, 0}\left(r_{0}(W), W, U\right)-Q_{Y, 0}\left(r_{0}(W), W\right)\right)\right. \\
& \left.\times\left(\mathbb{Q}_{Y, 0}\left(r_{0}(W), W, U\right)-Q_{Y, 0}^{*}\left(r_{0}(W), W\right)\right)\right] \\
& =E_{\mathbb{P}_{Q_{0}, g_{0}}}\left[\frac{\mathbf{1}\left\{A=r_{0}(W)\right\}}{Z}\left(\mathbb{Q}_{Y, 0}\left(r_{0}(W), W, U\right)-Q_{Y, 0}\left(r_{0}(W), W\right)\right)^{2}\right] \\
& +E_{\mathbb{P}_{Q_{0}, g_{0}}}\left[\frac{\mathbf{1}\left\{A=r_{0}(W)\right\}}{Z}\left(Q_{Y, 0}\left(r_{0}(W), W\right)-Q_{Y, 0}^{*}\left(r_{0}(W), W\right)\right)\right. \\
& \left.\times E_{\mathbb{P}_{Q_{0}, g_{0}}}\left(\mathbb{Q}_{Y, 0}\left(r_{0}(W), W, U\right)-Q_{Y, 0}\left(r_{0}(W), W\right) \mid W\right)\right] \\
& =E_{\mathbb{P}_{Q_{0}, g_{0}}}\left[\left(\mathbb{Q}_{Y, 0}\left(r_{0}(W), W, U\right)-Q_{Y, 0}\left(r_{0}(W), W\right)\right)^{2}\right. \\
& \left.\times E\left(\frac{\mathbf{1}\left\{A=r_{0}(W)\right\}}{Z} \mid W, U\right)\right] \\
& =E_{\mathbb{P}_{Q_{0}, g_{0}}}\left[\left(\mathbb{Q}_{Y, 0}\left(r_{0}(W), W, U\right)-Q_{Y, 0}\left(r_{0}(W), W\right)\right)^{2}\right]=\mathbb{P}_{Q_{0}, g_{0}} \chi_{0}^{2} .
\end{aligned}
$$

This completes the proof.

\section{APPENDIX B: TECHNICAL LEMMAS}

B.1. Lemmas for $M$ - and $Z$-estimation. The first lemma is a simple adaptation of [7, Corollary 3.2.3]. 
Lemma B.1. Let $\mathbf{M}_{n}$ and $\mathcal{M}_{n}$ be two real-valued stochastic processes indexed by a metric space $(\Theta, d)$. Consider a sequence of subsets $\Theta_{n} \subset \Theta$ and the following assumptions:

(a) For each $n \geq 1$, there exists $\tau_{n} \in \Theta$ such that, for all $\varepsilon>0$,

$$
\inf _{n \geq 1} \inf \left\{\mathcal{M}_{n}(\theta)-\mathcal{M}_{n}\left(\tau_{n}\right): \theta \in \Theta, d\left(\theta, \tau_{n}\right) \geq \varepsilon\right\}>0 .
$$

(b) For each $n \geq 1$, there exists $\tau_{n}^{*} \in \Theta_{n}$ such that $\mathcal{M}_{n}\left(\tau_{n}^{*}\right)=\inf _{\theta \in \Theta_{n}} \mathcal{M}_{n}(\theta)$. Moreover, $\mathcal{M}_{n}\left(\tau_{n}^{*}\right)-\mathcal{M}_{n}\left(\tau_{n}\right)=o_{P}(1)$.

(c) It holds that $\left\|\mathbf{M}_{n}-\mathcal{M}_{n}\right\|_{\Theta_{n}}=o_{P}(1)$.

Under (a), (b), and (c), if $\theta_{n} \in \Theta_{n}$ satisfies $\mathbf{M}_{n}\left(\theta_{n}\right)-\mathbf{M}_{n}\left(\tau_{n}^{*}\right) \leq 0$ for all $n \geq 1$, then $d\left(\theta_{n}, \tau_{n}\right)=o_{P}(1)$.

The corollary below will prove useful.

LemmA B.2. Let $\mathbf{Z}_{n}$ and $Z_{n}$ be two real-valued stochastic processes indexed by a metric space $(\Theta, d)$. Consider the following assumptions:

(d) For each $n \geq 1$, there exists $\tau_{n} \in \Theta$ such that $z_{n}\left(\tau_{n}\right)=0$ and, for all $\varepsilon>0$

$$
\inf _{n \geq 1} \inf \left\{\left|z_{n}(\theta)\right|: \theta \in \Theta, d\left(\theta, \tau_{n}\right) \geq \varepsilon\right\}>0 .
$$

(e) It holds that $\left\|\mathbf{Z}_{n}-z_{n}\right\|_{\Theta}=o_{P}(1)$.

Under (d) and (e), if $\theta_{n} \in \Theta$ satisfies $\mathbf{Z}_{n}\left(\theta_{n}\right)=0$ for all $n \geq 1$, then $d\left(\theta_{n}, \tau_{n}\right)=o_{P}(1)$.

Proof of Lemma B.1. Set $n \geq 1$. By $(\boldsymbol{a})$, it holds that

$$
\begin{aligned}
0 \leq & \mathcal{M}_{n}\left(\theta_{n}\right)-\mathcal{M}_{n}\left(t_{n}\right) \\
= & \left(\mathcal{M}_{n}\left(\theta_{n}\right)-\mathbf{M}_{n}\left(\theta_{n}\right)\right)+\left(\mathbf{M}_{n}\left(\theta_{n}\right)-\mathbf{M}_{n}\left(t_{n}^{*}\right)\right) \\
& \quad+\left(\mathbf{M}_{n}\left(t_{n}^{*}\right)-\mathcal{M}_{n}\left(t_{n}^{*}\right)\right)+\left(\mathcal{M}_{n}\left(t_{n}^{*}\right)-\mathcal{M}_{n}\left(t_{n}\right)\right) .
\end{aligned}
$$

The above first and third RHS terms are both upper-bounded by $\| \mathbf{M}_{n}-$ $\mathcal{M}_{n} \|_{\Theta_{n}}$. The second RHS term is non-positive by definition of $\theta_{n}$. The fourth RHS terms is $o_{P}(1)$ by (b). Thus, it actually holds that $0 \leq \mathcal{M}_{n}\left(\theta_{n}\right)-$ $\mathcal{M}_{n}\left(t_{n}\right) \leq 2\left\|\mathbf{M}_{n}-\mathcal{M}_{n}\right\|_{\Theta_{n}}+o_{P}(1)=o_{P}(1)$ by $(c)$.

Set $\varepsilon>0$. By $(\boldsymbol{a})$, there exists a positive random variable $\Delta$ which is independent of $n$ and such that $d\left(\theta_{n}, t_{n}\right) \geq \varepsilon$ implies $\mathcal{M}_{n}\left(\theta_{n}\right)-\mathcal{M}_{n}\left(t_{n}\right) \geq \Delta$ or, equivalently, $\Delta^{-1}\left[\mathcal{M}_{n}\left(\theta_{n}\right)-\mathcal{M}_{n}\left(t_{n}\right)\right] \geq 1$. Now, by Slutsky's lemma $[6$, Lemma 2.8], $\mathcal{M}_{n}\left(\theta_{n}\right)-\mathcal{M}_{n}\left(t_{n}\right)=o_{P}(1)$ entails $\Delta^{-1}\left[\mathcal{M}_{n}\left(\theta_{n}\right)-\mathcal{M}_{n}\left(t_{n}\right)\right]=$ $o_{P}(1)$. Therefore, we conclude that $d\left(\theta_{n}, t_{n}\right)=o_{P}(1)$ too. 
Proof of Lemma B.2. For all $n \geq 1$ and $\theta \in \Theta$, define $\Theta_{n}=\Theta, t_{n}^{*}=t_{n}$, $\mathbf{M}_{n}(\theta)=\left|\mathbf{Z}_{n}(\theta)\right|$ and $\mathcal{M}_{n}(\theta)=\left|\boldsymbol{Z}_{n}(\theta)\right|$. We note that $(\boldsymbol{a})$ in Lemma B.1 follows from $(\boldsymbol{d})$, that $(\boldsymbol{b})$ in Lemma B.1 trivially holds, and finally that (c) in Lemma B.1 is a consequence of $(e)$ and the reverse triangle inequality. Now, for each $n \geq 1, \mathbf{Z}_{n}\left(\theta_{n}\right)=0$ rewrites $\mathbf{M}_{n}\left(\theta_{n}\right)-\mathbf{M}_{n}\left(t_{n}^{*}\right) \leq 0$. Applying Lemma B.1 yields the result.

B.2. Maximal inequalities and convergence of empirical processes. The following two results are the cornerstones of our theoretical study.

Lemma B.3 (maximal inequality). Let $\mathcal{F}$ be a separable class of measurable, real-valued functions, with envelope function $F$. Set $n \geq 1$. It holds that

$$
E\left(\sqrt{n}\left\|P_{n}-P_{Q_{0}, \mathbf{g}_{n}}\right\|_{\mathcal{F}}\right) \lesssim J_{F}(1, \mathcal{F}) \times\|F\|_{2, P_{Q_{0}, g^{\mathrm{ref}}}} .
$$

LEMmA B.4 (convergence of empirical processes indexed by estimated functions). For each $n \geq 1$, let $\mathcal{F}_{n}=\left\{f_{\theta, \eta}: \theta \in \Theta, \eta \in T_{n}\right\}$ be a separable class of measurable, real-valued functions, with envelope function $F_{n}$. Suppose the following holds:

(a) The sequence $\left\{F_{n}\right\}_{n \geq 1}$ satisfies the Lindeberg condition: $\left\|F_{n}\right\|_{2, P_{Q_{0}, g} \text { ref }}=$ $O(1)$ and, for every $\delta>0,\left\|F_{n} \mathbf{1}\left\{F_{n}>\delta \sqrt{n}\right\}\right\|_{2, P_{Q_{0}, g} \text { ref }}=o(1)$.

(b) If $\delta_{n}=o(1)$, then it holds that $J_{F_{n}}\left(\delta_{n}, \mathcal{F}_{n}\right)=o(1)$.

If $\eta_{n} \in T_{n}$ is such that $\sup _{\theta \in \Theta}\left\|f_{\theta, \eta_{n}}-f_{\theta, \eta_{0}}\right\|_{2, P_{Q_{0}, g^{\mathrm{ref}}}}=o_{P}(1)$ for some $\eta_{0} \in \cap_{p \geq 1} \cup_{n \geq p} T_{n}$, then $\sup _{\theta \in \Theta}\left|\sqrt{n}\left(P_{n}-P_{Q_{0}, \mathbf{g}_{n}}\right)\left(f_{\theta, \eta_{n}}-f_{\theta, \eta_{0}}\right)\right|=o_{P}(1)$.

The proof of Lemma B.4 notably relies on the lemma below. Its proof, a straightforward adaptation of that of [8, Lemma 12], is omitted.

Lemma B.5. For each $n \geq 1$, let $\mathcal{F}_{n}$ be a class of measurable, realvalued functions with envelope function $F_{n}$ such that $\delta_{n}=o(1)$ implies $J_{F_{n}}\left(\delta_{n}, \mathcal{F}_{n}\right)=o(1)$. Then (i) $J_{F_{n}}\left(\delta, \mathcal{F}_{n}\right)=O(1)$ for every $\delta>0$, and (ii) for every $\varepsilon>0$, there exist $\delta>0$ and $n_{1} \geq 1$ such that $J_{F_{n}}\left(\delta, \mathcal{F}_{n}\right) \leq \varepsilon$ for all $n \geq n_{1}$.

Proof of Lemmas B.3 And B.4. The proofs of Lemmas B.3 and B.4 are best presented jointly.

Let us prove (B.1) from Lemma B.3 in three steps. 
Step one: decoupling. By [2, Proposition 6.1.5 and Remark 6.1.6], it is possible to enlarge the probability space and to define three sequences of random variables $\left\{\varepsilon_{n}\right\}_{n \geq 1},\left\{\left(O_{n}^{b}, Z_{n}^{b}\right)\right\}_{n \geq 1},\left\{\left(O_{n}^{\natural}, Z_{n}^{\natural}\right)\right\}_{n \geq 1}$ and a $\sigma$-field $\mathcal{G}$ such that

- $\left\{\varepsilon_{n}\right\}_{n \geq 1}$ is a sequence of independent Rademacher random variables, a sequence that is moreover independent of the three $\left\{\left(O_{n}, Z_{n}\right)\right\}_{n \geq 1}$, $\left\{\left(O_{n}^{b}, Z_{n}^{b}\right)\right\}_{n \geq 1},\left\{\left(O_{n}^{\natural}, Z_{n}^{\natural}\right)\right\}_{n \geq 1}$;

- the distributions of $\left(O_{1}^{b}, Z_{1}^{b}\right)$ and $\left(O_{1}^{\natural}, Z_{1}^{\natural}\right)$ coincide with that of $\left(O_{1}, Z_{1}\right)$ and, for every $n \geq 2$, the conditional distributions of $\left(O_{n}^{b}, Z_{n}^{b}\right)$ and $\left(O_{n}^{\natural}, Z_{n}^{\natural}\right)$ given $\mathcal{G}$ coincide with that of $\left(O_{n}, Z_{n}\right)$ given $\left(O_{1}, Z_{1}\right), \ldots$, $\left(O_{n-1}, Z_{n-1}\right)$;

- conditionally on $\mathcal{G}$, the two sequences $\left\{\left(O_{n}^{b}, Z_{n}^{b}\right)\right\}_{n \geq 1},\left\{\left(O_{n}^{\natural}, Z_{n}^{\natural}\right)\right\}_{n \geq 1}$ are independent and each with mutually independent elements.

The new sequences $\left\{\left(O_{n}^{b}, Z_{n}^{b}\right)\right\}_{n \geq 1}$ and $\left\{\left(O_{n}^{\natural}, Z_{n}^{\natural}\right)\right\}_{n \geq 1}$ are said "decoupled sequences" to $\left\{\left(O_{n}, Z_{n}\right)\right\}_{n \geq 1}$.

We denote $E_{\mathcal{G}}$ the conditional expectation given $\mathcal{G}$ and $E_{\mathcal{G}}^{b}$ the conditional expectation given $\mathcal{G}$ and $\left\{\left(O_{n}^{b}, Z_{n}^{b}\right)\right\}_{n \geq 1}$. We also characterize $P_{n}^{b}, P_{Q_{0}, \mathbf{g}_{n}}^{b}$ and $P_{n}^{0 b}$ by setting, for each $f: \mathcal{O} \times[0,1] \rightarrow \mathbb{R}, P_{n}^{b} f=n^{-1} \sum_{i=1}^{n} f\left(O_{i}^{b}, Z_{i}^{b}\right)$, $P_{Q_{0}, \mathbf{g}_{n}}^{b} f=n^{-1} \sum_{i=1}^{n} E_{\mathcal{G}}\left[f\left(O_{i}^{b}, Z_{i}^{b}\right)\right], P_{n}^{0 b} f=n^{-1} \sum_{i=1}^{n} \varepsilon_{i} f\left(O_{i}^{b}, Z_{i}^{b}\right)$.

Step two: symmetrizing. Let $\Phi$ be a non-decreasing, convex function mapping $\mathbb{R}_{+}$to $\mathbb{R}$. Set $n \geq 1$. By construction of the decoupled sequences, it holds that $E\left[\Phi\left(n\left\|P_{n}-P_{Q_{0}, \mathbf{g}_{n}}\right\|_{\mathcal{F}}\right)\right]=E\left(E_{\mathcal{G}}\left[\Phi\left(n\left\|P_{n}^{b}-P_{Q_{0}, \mathbf{g}_{n}}^{b}\right\|_{\mathcal{F}}\right)\right]\right)$. We now focus on $E_{\mathcal{G}}\left[\Phi\left(n\left\|P_{n}^{b}-P_{Q_{0}, \mathbf{g}_{n}}^{b}\right\|_{\mathcal{F}}\right)\right]$.

Note that

$$
\begin{aligned}
n\left\|P_{n}^{b}-P_{Q_{0}, \mathbf{g}_{n}}^{b}\right\|_{\mathcal{F}} & =\left\|\sum_{i=1}^{n} f\left(O_{i}^{b}, Z_{i}^{b}\right)-E_{\mathcal{G}}\left(f\left(O_{i}^{\natural}, Z_{i}^{\natural}\right)\right)\right\|_{\mathcal{F}} \\
& =\left\|\sum_{i=1}^{n} f\left(O_{i}^{b}, Z_{i}^{b}\right)-E_{\mathcal{G}}^{b}\left(f\left(O_{i}^{\natural}, Z_{i}^{\natural}\right)\right)\right\|_{\mathcal{F}} \\
& \leq E_{\mathcal{G}}^{b}\left[\left\|\sum_{i=1}^{n} f\left(O_{i}^{b}, Z_{i}^{b}\right)-f\left(O_{i}^{\natural}, Z_{i}^{\natural}\right)\right\|_{\mathcal{F}}\right],
\end{aligned}
$$

so that Jensen's inequality yields

$$
\Phi\left(n\left\|P_{n}^{b}-P_{Q_{0}, \mathbf{g}_{n}}^{b}\right\|_{\mathcal{F}}\right) \leq E_{\mathcal{G}}^{b}\left[\Phi\left(\left\|\sum_{i=1}^{n} f\left(O_{i}^{b}, Z_{i}^{b}\right)-f\left(O_{i}^{\natural}, Z_{i}^{\natural}\right)\right\|_{\mathcal{F}}\right)\right] .
$$


By taking outer (conditional) expectation, we obtain (B.2)

$$
E_{\mathcal{G}}\left[\Phi\left(n\left\|P_{n}^{b}-P_{Q_{0}, \mathbf{g}_{n}}^{b}\right\|_{\mathcal{F}}\right)\right] \leq E_{\mathcal{G}}\left[\Phi\left(\left\|\sum_{i=1}^{n} f\left(O_{i}^{b}, Z_{i}^{b}\right)-f\left(O_{i}^{\natural}, Z_{i}^{\natural}\right)\right\|_{\mathcal{F}}\right)\right] .
$$

Observe now that, for every $n$-tuple $\left(e_{1}, \ldots, e_{n}\right) \in\{-1,1\}^{n}$,

$$
\begin{aligned}
E_{\mathcal{G}}\left[\Phi \left(\| \sum_{i=1}^{n} f\left(O_{i}^{b}, Z_{i}^{b}\right)\right.\right. & -f\left(O_{i}^{\natural}, Z_{i}^{\natural}\right) \| \\
& =E_{\mathcal{G}}\left[\Phi\left(\left\|\sum_{i=1}^{n} e_{i}\left(f\left(O_{i}^{b}, Z_{i}^{b}\right)-f\left(O_{i}^{\natural}, Z_{i}^{\natural}\right)\right)\right\|_{\mathcal{F}}\right)\right]
\end{aligned}
$$

since, for each $1 \leq i \leq n,\left(O_{i}^{b}, Z_{i}^{b}\right)$ and $\left(O_{i}^{\natural}, Z_{i}^{\natural}\right)$ are independent and equal in law (conditional on $\mathcal{G}$ ). Consequently, (B.2) yields

$$
\begin{aligned}
& E_{\mathcal{G}}\left[\Phi\left(n\left\|P_{n}^{b}-P_{Q_{0}, \mathbf{g}_{n}}^{b}\right\|_{\mathcal{F}}\right)\right] \\
& \leq E_{\mathcal{G}}\left[\Phi\left(\left\|\sum_{i=1}^{n} \varepsilon_{i}\left(f\left(O_{i}^{b}, Z_{i}^{b}\right)-f\left(O_{i}^{\natural}, Z_{i}^{\natural}\right)\right)\right\|_{\mathcal{F}}\right)\right]
\end{aligned}
$$

where the expectation $E_{\mathcal{G}}$ to the right now also concerns the (conditionally and unconditionally on $\mathcal{G}$ ) independent $\left(\varepsilon_{1}, \ldots, \varepsilon_{n}\right)$. By the triangle inequality and convexity of $\Phi$, we see that the RHS expression of (B.3) is itself upper-bounded by

$$
\begin{aligned}
\frac{1}{2} E_{\mathcal{G}}\left[\Phi\left(2\left\|\sum_{i=1}^{n} \varepsilon_{i} f\left(O_{i}^{b}, Z_{i}^{b}\right)\right\|_{\mathcal{F}}\right)\right] & +\frac{1}{2} E_{\mathcal{G}}\left[\Phi\left(2\left\|\sum_{i=1}^{n} \varepsilon_{i} f\left(O_{i}^{\natural}, Z_{i}^{\natural}\right)\right\|_{\mathcal{F}}\right)\right] \\
& =E_{\mathcal{G}}\left[\Phi\left(2\left\|\sum_{i=1}^{n} \varepsilon_{i} f\left(O_{i}^{b}, Z_{i}^{b}\right)\right\|_{\mathcal{F}}\right)\right],
\end{aligned}
$$

hence

$$
E_{\mathcal{G}}\left[\Phi\left(n\left\|P_{n}^{b}-P_{Q_{0}, \mathbf{g}_{n}}^{b}\right\|_{\mathcal{F}}\right)\right] \leq E_{\mathcal{G}}\left[\Phi\left(2\left\|\sum_{i=1}^{n} \varepsilon_{i} f\left(O_{i}^{b}, Z_{i}^{b}\right)\right\|_{\mathcal{F}}\right)\right] .
$$

In conclusion, we derive the symmetrization inequality

$$
E\left[\Phi\left(n\left\|P_{n}-P_{Q_{0}, \mathbf{g}_{n}}\right\|_{\mathcal{F}}\right)\right] \leq E\left[\Phi\left(2 n\left\|P_{n}^{0 b}\right\|_{\mathcal{F}}\right)\right] .
$$


Step three: chaining. Taking $\Phi$ given by $\Phi(x)=x$ (all $x \geq 0)$ in (B.4) readily yields

$$
E\left(\sqrt{n}\left\|P_{n}-P_{Q_{0}, \mathbf{g}_{n}}\right\|_{\mathcal{F}}\right) \leq 2 E\left(\sqrt{n}\left\|P_{n}^{0 b}\right\|_{\mathcal{F}}\right) .
$$

Set now $\Phi(x)=\exp \left(x^{2}\right)-1($ all $x \geq 0)$ and let $\|\cdot\|_{\Phi}$ be the corresponding $\Phi$-Orlicz norm [7, page 95]. Conditionally on $\left(O_{1}^{b}, Z_{1}^{b}\right), \ldots,\left(O_{n}^{b}, Z_{n}^{b}\right)$, the process $\sqrt{n} P_{n}^{0 b}$ is sub-Gaussian for the $L^{2}\left(P_{n}^{b}\right)$-seminorm $\|\cdot\|_{2, n}^{b}$ by Hoeffding's inequality [7, Lemma 2.2.7]. The number $s_{n}^{b}=\sup _{f \in \mathcal{F}}\|f\|_{2, n}^{b}$ upper-bounds the radius of $\mathcal{F} \cup\{0\}$ wrt $\|\cdot\|_{2, n}^{b}$. Thus, by [7, Theorem 2.2.4] (a maximal inequality whose proof essentially relies on a chaining argument) and a change of variable, it holds that

$$
\begin{aligned}
\left\|\sqrt{n} P_{n}^{0 b}\right\|_{\Phi} & \lesssim \int_{0}^{s_{n}^{b}} \sqrt{1+\log N\left(\varepsilon, \mathcal{F}, L^{2}\left(P_{n}^{b}\right)\right)} d \varepsilon \\
& \leq\|F\|_{2, n}^{b} \int_{0}^{s_{n}^{b} /\|F\|_{2, n}^{b}} \sqrt{1+\log N\left(\varepsilon\|F\|_{2, n}^{b}, \mathcal{F}, L^{2}\left(P_{n}^{b}\right)\right)} d \varepsilon
\end{aligned}
$$

By definition of the uniform entropy integral, we therefore obtain

$$
\left\|\sqrt{n} P_{n}^{0 b}\right\|_{\Phi} \lesssim\|F\|_{2, n}^{b} J_{F_{n}}\left(s_{n}^{b} /\|F\|_{2, n}^{b}, \mathcal{F}\right),
$$

a result which holds conditionally on $\left(O_{1}^{b}, Z_{1}^{b}\right), \ldots,\left(O_{n}^{b}, Z_{n}^{b}\right)$. Finally, we take the expectation wrt to $\left(O_{1}^{b}, Z_{1}^{b}\right), \ldots,\left(O_{n}^{b}, Z_{n}^{b}\right)$ and note that $(a) s_{n}^{b} \leq\|F\|_{2, n}^{b}$, (b) $E\left(\|F\|_{2, n}^{b}\right) \lesssim\|F\|_{2, P_{Q_{0}, g} \text { ref }}$. In view of (B.5) this does yield

$$
\begin{aligned}
E\left(\sqrt{n}\left\|P_{n}-P_{Q_{0}, \mathbf{g}_{n}}\right\|_{\mathcal{F}}\right) & =E\left(\sqrt{n}\left\|P_{n}^{b}-P_{Q_{0}, \mathbf{g}_{n}}^{b}\right\|_{\mathcal{F}}\right) \\
& \lesssim E\left(\|F\|_{2, n}^{b} \times J_{F_{n}}\left(s_{n}^{b} /\|F\|_{2, n}^{b}, \mathcal{F}\right)\right) \\
& \leq J_{F_{n}}(1, \mathcal{F}) \times\|F\|_{2, P_{Q_{0}, g^{\mathrm{ref}}}},
\end{aligned}
$$

which completes the proof of (B.1).

We now show Lemma B.4. The proof follows closely that of [1, Part III, Theorem 6.16]. It has four steps.

Step one: preliminary. Introduce the classes $\widetilde{\mathcal{F}}_{n}^{0}$ (random) and $\mathcal{F}_{n}^{0}$ (deterministic) given by

$$
\widetilde{\mathcal{F}}_{n}^{0} \equiv\left\{f_{\theta, \eta_{n}}-f_{\theta, \eta_{0}}: \theta \in \Theta\right\} \subset \mathcal{F}_{n}^{0} \equiv\left\{f_{\theta, \eta}-f_{\theta, \eta_{0}}: \theta \in \Theta, \eta \in T_{n}\right\} .
$$

Lemma B.4 states that $\sqrt{n}\left\|P_{n}-P_{Q_{0}, \mathbf{g}_{n}}\right\|_{\widetilde{\mathcal{F}}_{n}^{0}}=o_{P}(1)$. 
For an arbitrarily fixed $\delta>0$, define

$$
\begin{aligned}
T_{n}^{0}(\delta) & \equiv\left\{\eta \in T_{n}: \sup _{\theta \in \Theta}\left\|f_{\theta, \eta}-f_{\theta, \eta_{0}}\right\|_{2, P_{Q_{0}, g^{\mathrm{ref}}}}^{2} \leq \delta^{2}\right\}, \\
\mathcal{F}_{n}^{0}(\delta) & \equiv\left\{f_{\theta, \eta}-f_{\theta, \eta_{0}}: \theta \in \Theta, \eta \in T_{n}^{0}(\delta)\right\} \subset \mathcal{F}_{n}^{0}, \\
\mathcal{F}_{n}^{0}(\delta)^{2} & \equiv\left\{h^{2}: h \in \mathcal{F}_{n}^{0}(\delta)\right\}, \quad \text { and } \\
s_{n}^{b}(\delta) & \equiv \frac{\sup _{h \in \mathcal{F}_{n}^{0}(\delta)}\|h\|_{2, n}^{b}}{\left\|1+2 F_{n}\right\|_{2, n}^{b}}=\frac{\left\|P_{n}^{b}\right\|_{\mathcal{F}_{n}^{0}(\delta)^{2}}}{\left\|1+2 F_{n}\right\|_{2, n}^{b}} .
\end{aligned}
$$

The classes $\widetilde{\mathcal{F}}_{n}^{0}, \mathcal{F}_{n}^{0}(\delta)$ and $\mathcal{F}_{n}^{0}$ admit $H_{n} \equiv 1+2 F_{n}$ as an envelope function. Because its definition involves $P_{n}^{b}, s_{n}^{b}(\delta)$ is random. Moreover, $\left\|H_{n}\right\|_{2, n}^{b} \geq 1$ and $\sup _{h \in \mathcal{F}_{n}^{0}(\delta)}\|h\|_{2, n}^{b} \leq\left\|2 F_{n}\right\|_{2, n}^{b}$ yield that

$$
s_{n}^{b}(\delta) \leq \min \left(1, \sup _{h \in \mathcal{F}_{n}^{0}(\delta)}\|h\|_{2, n}^{b}\right)=\min \left(1,\left\|P_{n}^{b}\right\|_{\mathcal{F}_{n}^{0}(\delta)^{2}}\right) .
$$

By (B.6) and the Cauchy-Schwarz inequality, we have

$$
\begin{aligned}
& {\left[E\left(\sqrt{n}\left\|P_{n}-P_{Q_{0}, \mathbf{g}_{n}}\right\|_{\mathcal{F}_{n}^{0}(\delta)}\right)\right]^{2}} \\
& \quad \lesssim \quad\left[E\left(\left\|H_{n}\right\|_{2, n}^{b} \times J_{H_{n}}\left(s_{n}^{b}(\delta), \mathcal{F}_{n}^{0}(\delta)\right)\right)\right]^{2} \\
& \quad \leq E\left(\left\|H_{n}\right\|_{2, n}^{b 2}\right) \times E\left(J_{H_{n}}\left(s_{n}^{b}(\delta), \mathcal{F}_{n}^{0}(\delta)\right)^{2}\right)
\end{aligned}
$$

Step two: studying $s_{n}^{b}(\delta)$. We now show that there exists an integer $n_{1}(\delta)$ such that $E\left(s_{n}^{b}(\delta)\right) \lesssim \min \left(1, \delta^{2}\right)$ for all $n \geq n_{1}(\delta)$. The proof is based on (B.7) and the decomposition $\mathcal{F}_{n}^{0}(\delta)^{2}=\mathcal{F}_{n, 1}^{0}(\delta)^{2} \cup \mathcal{F}_{n, 2}^{0}(\delta)^{2}$ for

$$
\begin{aligned}
& \mathcal{F}_{n, 1}^{0}(\delta)^{2} \equiv\left\{h^{2} \mathbf{1}\left\{2 F_{n} \leq \rho \sqrt{n} / 2\right\}: h \in \mathcal{F}_{n}^{0}(\delta)\right\} \\
& \mathcal{F}_{n, 2}^{0}(\delta)^{2} \equiv\left\{h^{2} \mathbf{1}\left\{2 F_{n}>\rho \sqrt{n} / 2\right\}: h \in \mathcal{F}_{n}^{0}(\delta)\right\}
\end{aligned}
$$

where the constant $\rho>0$ will be determined later.

Obviously, $\rho \sqrt{n} / 2 \times 2 F_{n}=\rho \sqrt{n} F_{n}$ is an envelope function for $\mathcal{F}_{n, 1}^{0}(\delta)^{2}$. By (B.6), we thus have

$$
\begin{aligned}
E\left(\sqrt{n}\left\|P_{n}^{b}-P_{Q_{0}, \mathbf{g}_{n}}^{b}\right\|_{\mathcal{F}_{n, 1}^{0}(\delta)^{2}}\right) & \\
& \lesssim J_{\rho \sqrt{n} F_{n}}\left(1, \mathcal{F}_{n, 1}^{0}(\delta)^{2}\right) \times\left\|\rho \sqrt{n} F_{n}\right\|_{2, P_{Q_{0}, g^{\text {ref }}}}
\end{aligned}
$$


But $J_{\rho \sqrt{n} F_{n}}\left(1, \mathcal{F}_{n, 1}^{0}(\delta)^{2}\right)$ easily compares to $J_{F_{n}}\left(1, \mathcal{F}_{n}\right)$. Indeed, whichever are $\varepsilon>0, h, h^{\prime} \in \mathcal{F}_{n}^{0}(\delta)$, and $m$ a discrete probability measure such that $0<m F_{n}$, it holds that

$$
m\left(h^{2}-h^{\prime 2}\right)^{2} \mathbf{1}\left\{2 F_{n} \leq \rho \sqrt{n} / 2\right\} \leq\left(4 F_{n}\right)^{2} m\left(h-h^{\prime}\right)^{2} \leq(\rho \sqrt{n})^{2} m\left(h-h^{\prime}\right)^{2},
$$

hence

$$
N\left(\varepsilon\left\|\rho \sqrt{n} F_{n}\right\|_{m, 2}, \mathcal{F}_{n, 1}^{0}(\delta)^{2}\right) \leq N\left(\varepsilon\left\|F_{n}\right\|_{m, 2}, \mathcal{F}_{n}\right),
$$

from which we deduce that $J_{\rho \sqrt{n} F_{n}}\left(1, \mathcal{F}_{n, 1}^{0}(\delta)^{2}\right) \leq J_{F_{n}}\left(1, \mathcal{F}_{n}\right)$. This bound and (B.9) yield

$$
E\left(\left\|P_{n}^{b}-P_{Q_{0}, \mathbf{g}_{n}}^{b}\right\|_{\mathcal{F}_{n, 1}^{0}(\delta)^{2}}\right) \lesssim \rho J_{F_{n}}\left(1, \mathcal{F}_{n}\right) \times\left\|F_{n}\right\|_{2, P_{Q_{0}, g^{\mathrm{ref}}}} .
$$

Furthermore, because (i) $2 F_{n}$ is an envelope function for $\mathcal{F}_{n, 2}^{0}(\delta)^{2}$ and (ii) the design $\mathbf{g}_{n}$ attached to the sequence $\left\{\left(O_{n}^{b}, Z_{n}^{b}\right)\right\}_{n \geq 1}$ is bounded away from 0 and 1 , it holds that

$$
E\left(\left\|P_{n}^{b}-P_{Q_{0}, \mathbf{g}_{n}}^{b}\right\|_{\mathcal{F}_{n, 2}^{0}(\delta)^{2}}\right) \lesssim \rho J_{F_{n}}\left(1, \mathcal{F}_{n}\right) \times\left\|F_{n} \mathbf{1}\left\{F_{n}>\rho \sqrt{n} / 2\right\}\right\|_{2, P_{Q_{0}, g^{\text {ref }}}} .
$$

Since $\mathcal{F}_{n}^{0}(\delta)^{2}$ is the union of $\mathcal{F}_{n, 1}^{0}(\delta)^{2}$ and $\mathcal{F}_{n, 2}^{0}(\delta)^{2}$, the previous inequality combined with (B.10) then yields

$$
\begin{aligned}
E\left(\left\|P_{n}^{b}-P_{Q_{0}, \mathbf{g}_{n}}^{b}\right\|_{\mathcal{F}_{n}^{0}(\delta)^{2}}\right) & \\
& \lesssim \rho J_{F_{n}}\left(1, \mathcal{F}_{n}\right) \times\left\|F_{n}\right\|_{2, P_{Q_{0}, g^{\mathrm{ref}}}} \\
& \quad+\rho J_{F_{n}}\left(1, \mathcal{F}_{n}\right) \times\left\|F_{n} \mathbf{1}\left\{F_{n}>\rho \sqrt{n} / 2\right\}\right\|_{2, P_{Q_{0}, g^{\mathrm{ref}}}} .
\end{aligned}
$$

By (a) in Lemma B.4, it holds that $\left\|F_{n}\right\|_{2, P_{Q_{0}, g^{\mathrm{ref}}}}=O(1)$ and $\| F_{n} \mathbf{1}\left\{F_{n}>\right.$ $\rho \sqrt{n} / 2\} \|_{2, P_{Q_{0}, g^{\mathrm{ref}}}}=o(1)$. By Lemma B.5, $J_{F_{n}}\left(1, \mathcal{F}_{n}\right)=O(1)$. Therefore, it is possible to choose $\rho>0$ and find $n_{1}(\delta) \geq 1$ such that, for all $n \geq n_{1}(\delta)$,

$$
E\left(\left\|P_{n}^{b}-P_{Q_{0}, \mathbf{g}_{n}}^{b}\right\|_{\mathcal{F}_{n}^{0}(\delta)^{2}}\right) \leq \delta^{2}
$$

Now, the definition of $\mathcal{F}_{n}^{0}(\delta)$ and the above remark (ii) about the design $\mathbf{g}_{n}$ yield the additional inequality, valid for all sample size:

$$
E\left(\left\|P_{Q_{0}, \mathbf{g}_{n}}^{b}\right\|_{\mathcal{F}_{n}^{0}(\delta)^{2}}\right) \lesssim \delta^{2}
$$

By the triangle inequality, (B.7), (B.11) and (B.12) imply

$$
E\left(s_{n}^{b}(\delta)\right) \leq \min \left(1, E\left(\left\|P_{n}^{b}\right\|_{\mathcal{F}_{n}^{0}(\delta)^{2}}\right)\right) \lesssim \min \left(1, \delta^{2}\right)
$$


for all $n \geq n_{1}(\delta)$. Markov's inequality then yields that, for all $\xi>0$ and $n \geq n_{1}(\delta)$,

$$
P\left(s_{n}^{b}(\delta) \geq \xi\right) \leq \xi^{-1} \min \left(1, \delta^{2}\right) .
$$

This completes the study of $s_{n}^{b}(\delta)$.

Step three: fine-tuning. Set arbitrarily $\alpha, \varepsilon>0$. Note that the above remark (ii) about the design $\mathbf{g}_{n}$ and assumption (a) in Lemma B.4 imply the existence of a constant $C_{1}>0$ such that the following bounds hold on the leftmost factor of the RHS expression in (B.8):

$$
E\left(\left\|H_{n}\right\|_{2, n}^{22}\right) \lesssim\left\|F_{n}\right\|_{2, P_{Q_{0}, g \text { ref }}}^{2} \leq C_{1}^{2}
$$

By assumption (b) in Lemma B.4 and Lemma B.5, there exist $0<\xi \leq 1$, $C_{2}>0$ and $n_{2} \geq 1$ such that $J_{H_{n}}\left(\xi, \mathcal{F}_{n}\right) \leq \alpha \varepsilon / C_{1}$ and $J_{H_{n}}\left(1, \mathcal{F}_{n}\right)^{2} \leq C_{2}^{2}$ for all $n \geq n_{2}$. Let $\delta_{0}>0$ be such that $\delta_{0} \leq \alpha \varepsilon \sqrt{3 \xi} / C_{1} C_{2}$. By assumption on $\eta_{n}$ in Lemma B.4, we know that there exists $n_{3}\left(\delta_{0}\right) \geq 1$ such that $P\left(\eta_{n} \notin\right.$ $\left.T_{n}^{0}\left(\delta_{0}\right)\right) \leq \varepsilon$ for all $n \geq n_{3}\left(\delta_{0}\right)$.

Step four: wrapping up. Let $n$ be larger than $\max \left(n_{1}\left(\delta_{0}\right), n_{2}, n_{3}\left(\delta_{0}\right)\right)$. It holds that

$$
\begin{aligned}
A \equiv P & \left(\sup _{\theta \in \Theta}\left|\sqrt{n}\left(P_{n}-P_{Q_{0}, \mathbf{g}_{n}}\right)\left(f_{\theta, \eta_{n}}-f_{\theta, \eta_{0}}\right)\right| \geq \alpha\right) \\
& =P\left(\sqrt{n}\left\|P_{n}-P_{Q_{0}, \mathbf{g}_{n}}\right\|_{\widetilde{\mathcal{F}}_{n}^{0}} \geq \alpha\right) \\
& \leq P\left(\eta_{n} \notin T_{n}^{0}\left(\delta_{0}\right)\right)+P\left(\eta_{n} \in T_{n}^{0}\left(\delta_{0}\right), \sqrt{n}\left\|P_{n}-P_{Q_{0}, \mathbf{g}_{n}}\right\|_{\widetilde{\mathcal{F}}_{n}^{0}} \geq \alpha\right) \\
& \leq \varepsilon+P\left(\sqrt{n}\left\|P_{n}-P_{Q_{0}, \mathbf{g}_{n}}\right\|_{\mathcal{F}_{n}^{0}\left(\delta_{0}\right)} \geq \alpha\right) .
\end{aligned}
$$

By Markov's inequality, (B.8), (B.14) and (B.13), we obtain the inequalities

$$
\begin{aligned}
A & \leq \varepsilon+\alpha^{-1} E\left(\left\|H_{n}\right\|_{2, n}^{b 2}\right)^{1 / 2} \times E\left(J_{H_{n}}\left(s_{n}^{b}(\delta), \mathcal{F}_{n}^{0}(\delta)\right)^{2}\right)^{1 / 2} \\
& \leq \varepsilon+\alpha^{-1} C_{1} \times\left(P\left(s_{n}^{b}\left(\delta_{0}\right) \geq \xi\right) \times J_{H_{n}}\left(1, \mathcal{F}_{n}\right)^{2}+J_{H_{n}}\left(\xi, \mathcal{F}_{n}\right)^{2}\right)^{1 / 2} \\
& \leq \varepsilon+\alpha^{-1} C_{1} \times\left(\xi^{-1} \min \left(1, \delta_{0}^{2}\right) \times C_{2}^{2}+\left(C_{1}^{-1} \alpha \varepsilon\right)^{2}\right) \leq 3 \varepsilon .
\end{aligned}
$$

Since $\alpha$ and $\varepsilon$ were arbitrarily chosen, this completes the proof of Lemma B.4.

\section{APPENDIX C: PATHWISE DIFFERENTIABILITY}

The next two lemmas are summaries of results stated and shown in $[3,4]$. We state them for the sake of completeness. 
Lemma C.1. Set $\rho \in \mathcal{R}$, a known TR. Let $\Psi_{\rho}: \mathcal{M} \rightarrow[0,1]$ be given by

$$
\Psi_{\rho}\left(P_{Q, g}\right) \equiv E_{Q}\left(Q_{Y}(\rho(W), W)\right) .
$$

The mapping $\Psi_{\rho}: \mathcal{M} \rightarrow[0,1]$ is pathwise differentiable at every $P_{Q, g} \in$ $\mathcal{M}$ with respect to (wrt) the maximal tangent space. Its efficient influence curve at $P_{Q, g}$ is $D_{\rho}(Q, g)$ which satisfies $D_{\rho}(Q, g)(O)=D_{W, \rho}(Q, g)(W)+$ $D_{Y, \rho}(Q, g)(O)$ with

$$
\begin{aligned}
D_{W, \rho}(Q)(W) & \equiv Q_{Y}(\rho(W), W)-\Psi_{\rho}\left(P_{Q, g}\right), \\
D_{Y, \rho}(Q, g)(O) & \equiv \frac{\mathbf{1}\{A=\rho(W)\}}{g(A \mid W)}\left(Y-Q_{Y}(A, W)\right) .
\end{aligned}
$$

The variance $\operatorname{Var}_{P_{Q, g}} D_{\rho}(Q, g)(O)$ is a Cramér-Rao lower bound for the asymptotic variance of any regular and asymptotically linear estimator of $\Psi_{\rho}\left(P_{Q, g}\right)$ when sampling independently from $P_{Q, g}$.

In addition, if $g=g^{\prime}$, then $E_{Q, g}\left(D_{\rho}\left(Q^{\prime}, g^{\prime}\right)(O)\right)=0$ implies $\Psi_{\rho}\left(P_{Q^{\prime}, g^{\prime}}\right)=$ $\Psi_{\rho}\left(P_{Q, g}\right)$.

The notation $D_{W, \rho}(Q)$ conveys the notion that the first component of $D_{\rho}(Q, g)$ does not depend on $g$. This is true because $\Psi_{\rho}\left(P_{Q, g}\right)$ does not depend on $g$ either.

Lemma C.2. The mapping $\Psi: \mathcal{M} \rightarrow[0,1]$ is pathwise differentiable at every $P_{Q, g} \in \mathcal{M}$ wrt the maximal tangent space. Its efficient influence curve at $P_{Q, g}$ is $D(Q, g)$ which satisfies $D(Q, g)(O)=D_{W}(Q, g)(W)+$ $D_{Y}(Q, g)(O)$ with

$$
\begin{aligned}
D_{W}(Q)(W) & \equiv Q_{Y}\left(r\left(Q_{Y}\right)(W), W\right)-\Psi\left(P_{Q, g}\right), \\
D_{Y}(Q, g)(O) & \equiv \frac{\mathbf{1}\left\{A=r\left(Q_{Y}\right)(W)\right\}}{g(A \mid W)}\left(Y-Q_{Y}(A, W)\right) .
\end{aligned}
$$

The variance $\operatorname{Var}_{P_{Q, g}} D(Q, g)(O)$ is a Cramér-Rao lower bound for the asymptotic variance of any regular and asymptotically linear estimator of $\Psi\left(P_{Q, g}\right)$ when sampling independently from $P_{Q, g}$.

In addition, if $g=g^{\prime}$, then $E_{Q, g}\left(D\left(Q^{\prime}, g^{\prime}\right)(O)\right)=0$ implies

$$
\Psi\left(P_{Q^{\prime}, g^{\prime}}\right)=E_{Q}\left(Q_{Y}\left(r\left(Q_{Y}^{\prime}\right)(W), W\right)\right) .
$$

In particular, if $r\left(Q_{Y}\right)=r\left(Q_{Y}^{\prime}\right)$ and $g=g^{\prime}$, then $E_{Q, g}\left(D\left(P_{Q^{\prime}, g^{\prime}}\right)(O)\right)=0$ implies $\Psi\left(P_{Q^{\prime}, g^{\prime}}\right)=\Psi\left(P_{Q, g}\right)$.

APPENDIX D: A FIGURE AND A TABLE SUMMARIZING THE SIMULATION STUDY 

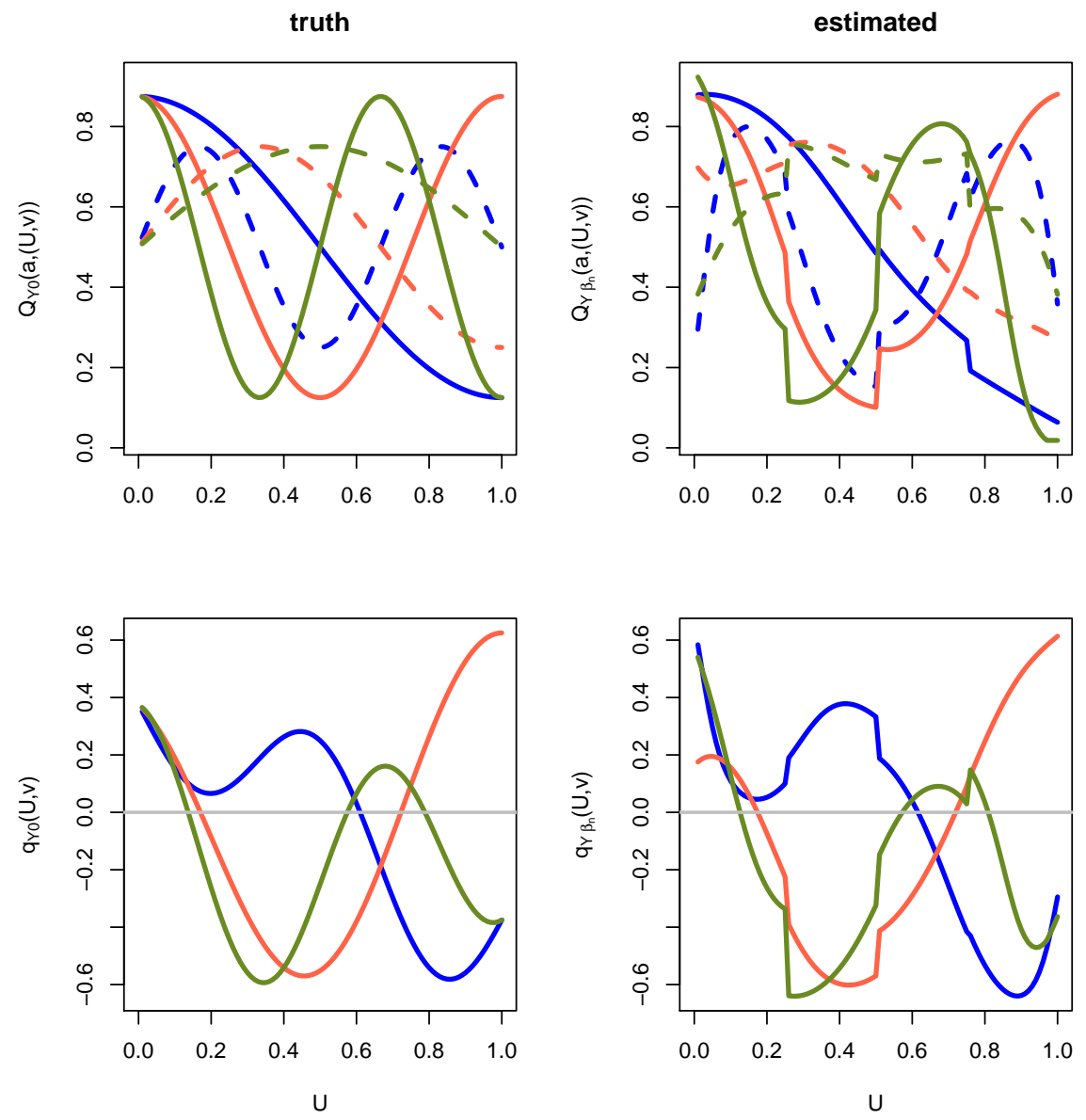

FIGURE 2. Illustrating the data-adaptive inference of the optimal treatment rule, its mean reward and the related pseudo-regrets through the representation of the conditional mean $Q_{Y, 0}$, blip function $q_{Y, 0}$ and their estimators (see also Figure 1). Top left plot. The solid curves represent $U \mapsto Q_{Y, 0}(1,(U, v)$ ) for $v=1$ (in blue, minimum reached at $U=1$ ), $v=2$ (in pink, minimum reached at $U=1 / 2$ ) and $v=3$ (in green, minimum reached at $U=1 / 3)$. The dashed curves represent $U \mapsto Q_{Y, 0}(0,(U, v))$ for $v=1$ (in blue, maximum reached at $U=1 / 6$ ), $v=2$ (in pink, maximum reached at $U=1 / 3$ ) and $v=3$ (in green, minimum reached at $U=1 / 2$ ). Bottom left plot. The curves represent $U \mapsto q_{Y, 0}(U, v)$ for $v=1$ (in blue, minimum reached close to $1 / 9$ ), $v=2$ (in pink, minimum reached close to $1 / 2$ ) and $v=3$ (in green, minimum reached close to $1 / 3)$. Right plots. Counterparts to the left plots, where $Q_{Y, 0}$ and $q_{Y, 0}$ are replaced with $Q_{Y, \beta_{n}}$ and $q_{Y, \beta_{n}}$ for $n=1000$, the final sample size. 


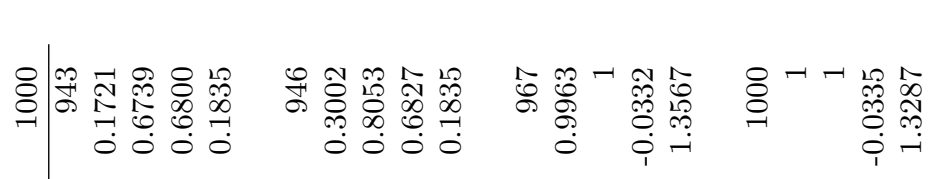

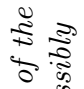

क \&

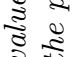

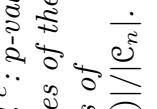

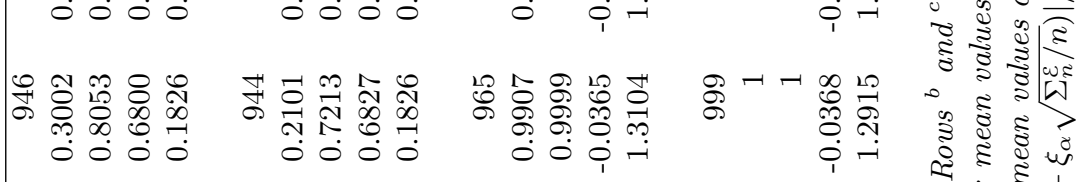

ஓ

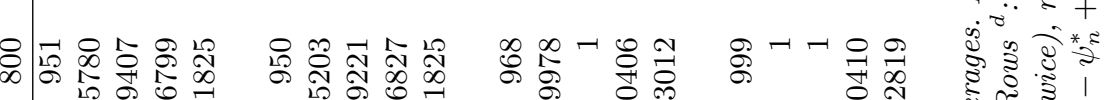

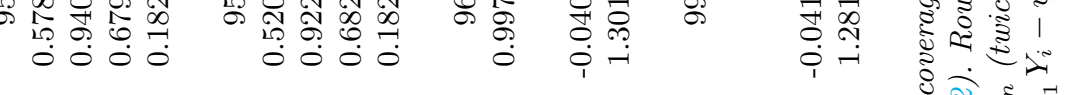

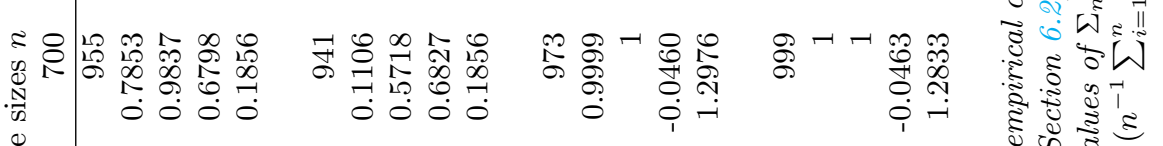

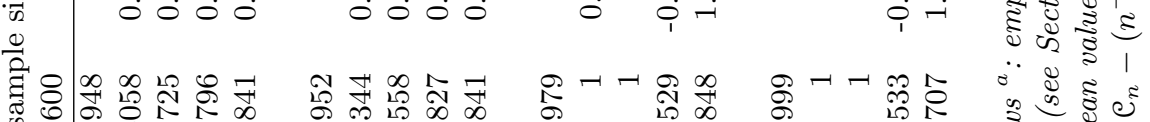

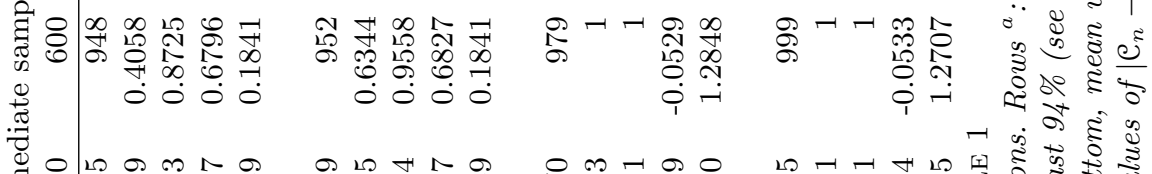

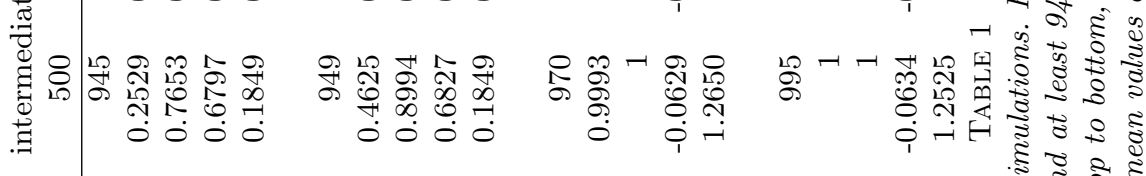

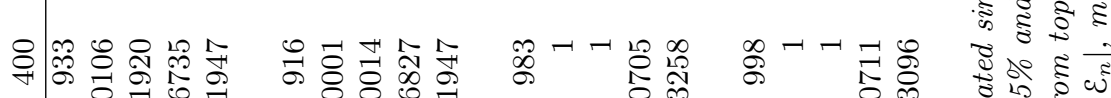

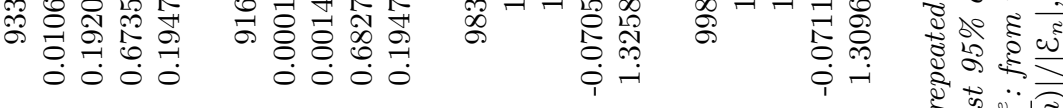

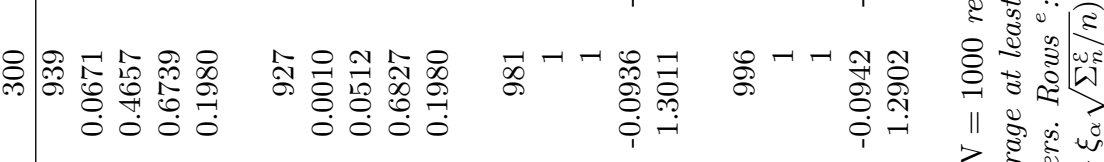

\&

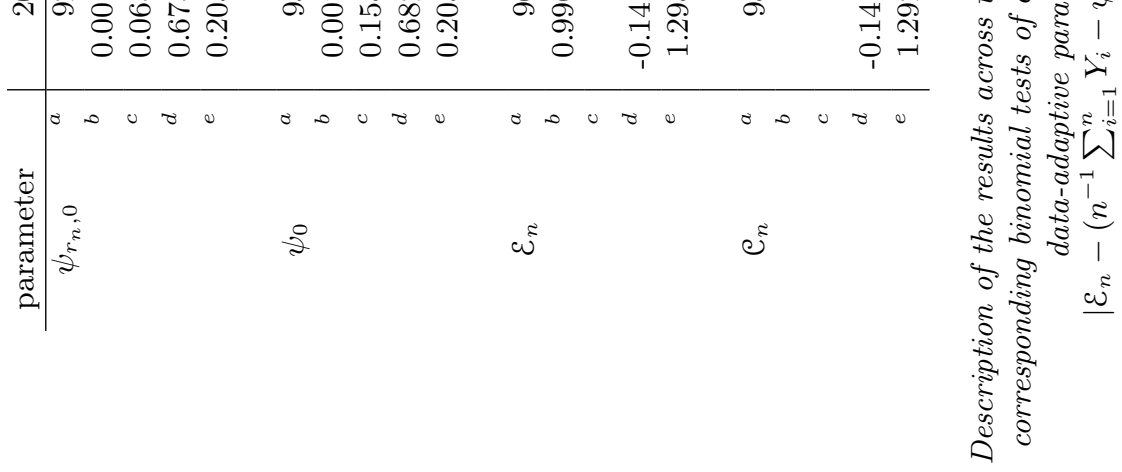




\section{APPENDIX E: NOTATION INDEX}

This notation index covers the entirety of the main article and gives the bare minimum to skim through the pages of the supplemental article.

\begin{tabular}{|c|c|}
\hline$\equiv$ & equal, by definition, to \\
\hline$\|\cdot\|_{\infty}$ & supremum norm \\
\hline$\|\cdot\|_{2, \mu}$ & $L^{2}(\mu)$-norm \\
\hline$\|\cdot\|_{2, P_{Q_{0}, g^{\mathrm{ref}}}}$ & $L^{2}\left(P_{Q_{0}, g^{\mathrm{ref}}}\right)$-norm \\
\hline$\|f\|_{p}$ & seminorm of $f: \mathcal{W} \rightarrow \mathbb{R}$ given by \\
\hline & $\|f\|_{p}^{p} \equiv E_{Q_{0}}\left(\left|q_{Y, 0}(W)\right| \times|f(W)|^{p}\right)$ \\
\hline$\left\|P_{n}-P_{Q_{0}, \mathbf{g}_{n}}\right\|_{\mathcal{F}}$ & $\begin{array}{l}\text { the supremum of }\left|\left(P_{n}-P_{Q_{0}, \mathbf{g}_{n}}\right) f\right| \text { in } f \in \mathcal{F} \text {, with } \mathcal{F} \text { a } \\
\text { separable set of measurable functions over } \mathcal{O}\end{array}$ \\
\hline$\beta, \beta_{n, 0}, \beta_{0}, \beta_{n}$ & see $Q_{Y, \beta}, Q_{Y, \beta_{n, 0}}, Q_{Y, \beta_{0}}, Q_{Y, \beta_{n}}$ \\
\hline blip function & $\begin{array}{l}\text { generically denoted } q_{Y} \text {, a function describing the ex- } \\
\text { pected difference in reward under a "blip" in treatment, } \\
\text { conditional on baseline covariates }\end{array}$ \\
\hline $\mathrm{C}_{n}$ & $\begin{array}{l}\text { counterfactual cumulative pseudo-regret at sample size } n \text {, } \\
\text { see }(5.5)\end{array}$ \\
\hline$\Delta\left(g_{n}, g_{0}\right)$ & a measure of discrepancy between $g_{n}$ and $g_{0}$, see (3.5) \\
\hline$\Delta\left(r_{n}, r_{0}\right)$ & a measure of discrepancy between $r_{n}$ and $r_{0}$, see (3.3) \\
\hline$d_{W, 0}^{*}, d_{Y, 0}^{*}$ & $\begin{array}{l}\text { two components of the influence function in the asymp- } \\
\text { totic linear expansion (4.15) of } \psi_{n}^{\star}-\psi_{r_{n}, 0} \text {, see (4.9) and } \\
\text { (4.10) }\end{array}$ \\
\hline$d_{W, n}^{*}, d_{Y, n}^{*}$ & $\begin{array}{l}\text { empirical counterparts to } d_{W, 0}^{*} \text { and } d_{Y, 0}^{*} \text {, see }(4.12) \text { and } \\
(4.13)\end{array}$ \\
\hline$D_{r_{0}}\left(Q_{0}^{\star}, g_{0}\right)$ & efficient influence curve of $\Psi_{r_{0}}$ at $P_{Q_{0}^{\star}, g_{0}}$, see Lemma C.1 \\
\hline$\epsilon_{0}(\rho)$ & optimal fluctuation parameter along \\
\hline & $\left\{Q_{Y, \beta_{0}, g_{0}, \rho}(\epsilon): \epsilon \in \mathcal{E}\right\}$, see $(4.3)$ and $\mathbf{A} \mathbf{3}$ \\
\hline$\epsilon_{n}$ & $\begin{array}{l}\text { empirically optimal fluctuation parameter along } \\
\left\{Q_{Y, \beta_{n}, g_{n}, r_{n}}(\epsilon): \epsilon \in \mathcal{E}\right\} \text {, see }(2.15) \text { and }(2.16)\end{array}$ \\
\hline$\varepsilon_{n}$ & $\begin{array}{l}\text { empirical cumulative pseudo-regret at sample size } n \text {, see } \\
(5.2)\end{array}$ \\
\hline$F, F_{n}$ & envelope functions \\
\hline$\gamma_{1}, \gamma_{2}$ & $\begin{array}{l}\text { positive constants involved in the statement of } \mathbf{A} \mathbf{5}, \mathbf{A} \mathbf{5}^{*} \\
\text { and } \mathbf{A} \mathbf{5}^{* *}\end{array}$ \\
\hline
\end{tabular}


$g$

$g^{\mathrm{b}}$

$g^{\text {ref }}$

$g_{0}$

$g_{i}, g_{n}$

$\mathbf{g}_{n}$

$G_{n}$

$\mathcal{G}$

$\mathcal{G}_{1, n}, \mathcal{G}_{1}$

$H_{\rho}(g)(O)$

$J_{F}(\delta, \mathcal{F})$

$L, L^{\mathrm{kl}}, L^{\mathrm{ls}}$

$L\left(\mathcal{Q}_{1, n}\right)$

$\mathcal{M}$

$N\left(\delta, \mathcal{F},\|\cdot\|_{2, \mu}\right)$

$\mathcal{O}$

$\mathbf{O}_{n}$ generic stochastic TR such that, under $g$, the conditional distribution of $A$ given $W$ is the Bernoulli law with parameter $g(1 \mid W)=1-g(0 \mid W) \in(0,1)$

balanced stochastic TR, given by $g^{\mathrm{b}}(1 \mid W)=g^{\mathrm{b}}(0 \mid W)=$ $1 / 2$

stochastic TR bounded away from 0 and 1 serving as a reference

stochastic TR defined as an approximation to $r_{0}$, see (3.1)

data-adaptive stochastic TRs used to assign treatment to the $i$ th and $n$th patients, see (2.11)

ordered vector $\mathbf{g}_{n} \equiv\left(g_{1}, \ldots, g_{n}\right)$ of the $n$ first dataadaptive stochastic TRs

non-decreasing, $c_{n}$-Lipschitz approximation to $x \mapsto$ $\mathbf{1}\{x \geq 0\}$ on $[-1,1]$ taking values in $\left[t_{n}, 1-t_{n}\right] \subset[0,1]$ and such that $t_{n} \downarrow t_{\infty}>0$ and $c_{n} \uparrow c_{\infty}<\infty$ see (2.5)

semiparametric collection of all $g \mathrm{~s}$

the subsets of $\mathcal{G}$ defined as $\mathcal{G}_{1, n} \equiv\left\{G_{n}\left(q_{Y}\right): Q_{Y} \in \mathcal{Q}_{1, n}\right\}$ and $\mathcal{G}_{1} \equiv \cup_{n \geq 1} \mathcal{G}_{1, n}$

clever covariate associated with $\mathrm{TR} \rho \in \mathcal{R}$ and stochastic TR $g \in \mathcal{G}$, see (2.14): $H_{\rho}(g)(O) \equiv \frac{\mathbf{1}\{A=\rho(W)\}}{g(A \mid W)}$; it is used to fluctuate an initial estimator of $Q_{Y, 0}$

the uniform entropy integral at $\delta$ of $\mathcal{F}$ wrt an envelope function $F$, i.e., $\int_{0}^{\delta} \sqrt{\log \sup _{\mu} N\left(\varepsilon\|F\|_{2, \mu}, \mathcal{F},\|\cdot\|_{2, \mu}\right)} d \varepsilon$, with a supremum taken over all probability measures $\mu$ for which $\|F\|_{2, \mu}>0$, a measure of complexity of $\mathcal{F}$

loss functions for the estimation of $Q_{Y, 0}$, where the quasi negative-log-likelihood and least-square loss functions $L^{\mathrm{kl}}$ and $L^{\mathrm{ls}}$ are given by (2.6) and (2.7)

given a loss function $L$, the set $\left\{L\left(Q_{Y, \beta}\right): Q_{Y, \beta} \in \mathcal{Q}_{1, n}\right\}$

semiparametric model $\mathcal{M} \equiv\left\{P_{Q, g}: Q \in \mathcal{Q}, g \in \mathcal{G}\right\}$

$\delta$-covering number of $\mathcal{F}$ wrt $\|\cdot\|_{2, \mu}$

bounded space $\mathcal{O} \equiv \mathcal{W} \times\{0,1\} \times(0,1)$ where a generic observed data-structure takes its values

ordered vector $\mathbf{O}_{n} \equiv\left(O_{1}, \ldots, O_{n}\right)$ of the $n$ first observed data-structures $O_{i} \equiv\left(W_{i}, A_{i}, Y_{i}\right)$ 


\begin{tabular}{|c|c|}
\hline$P_{n}$ & the empirical distribution of $\mathbf{O}(n)$ \\
\hline$P_{n} f$ & shorthand notation for $n^{-1} \sum_{i=1}^{n} f\left(O_{i}, Z_{i}\right)$ \\
\hline$P_{Q, g}$ & $\begin{array}{l}\text { distribution of } O \equiv(W, A, Y) \text { obtained by drawing } W \\
\text { from its marginal distribution encoded in } Q \text {, then } A \text { from } \\
\text { the Bernoulli distribution with parameter } g(1 \mid W) \text {, then } \\
Y \text { from its conditional distribution given }(A, W) \text { encoded } \\
\text { in } Q\end{array}$ \\
\hline$P_{Q, g} f$ & shorthand notation for $E_{Q, g}(f(O, Z))$ \\
\hline$P_{Q_{0}, g_{i}}$ & $\begin{array}{l}\text { same as } P_{Q, g} \text { with } Q=Q_{0} \text { and } g \equiv g_{i} \text {, the conditional } \\
\text { distribution of } O_{i} \text { given } \mathbf{O}(i-1)\end{array}$ \\
\hline$P_{Q_{0}, g_{i}} f$ & shorthand notation for $E_{Q_{O}, g_{i}}\left[f\left(O_{i}, Z_{i}\right) \mid \mathbf{O}(i-1)\right]$ \\
\hline$P_{Q_{0}, \mathbf{g}_{n}} f$ & shorthand notation for $n^{-1} \sum_{i=1}^{n} P_{Q_{0}, g_{i}} f$ \\
\hline$\psi_{0}$ & mean reward of the optimal TR under $Q_{0}$, see (2.3) \\
\hline$\psi_{r_{n}, 0}$ & $\begin{array}{l}\text { a data-adaptive parameter, the mean reward of empirical } \\
\text { TR } r_{n} \text { under } Q_{0} \text {, see }(4.4)\end{array}$ \\
\hline$\psi_{n}^{*}$ & $\begin{array}{l}\text { targeted minimum loss estimator (TMLE) of } \psi_{r_{n}, 0} \text { at } \\
\text { sample size } n \text {, see }(2.17)\end{array}$ \\
\hline$\Psi$ & $\begin{array}{l}\text { mapping from } \mathcal{M} \text { to }[0,1] \text { given by }(2.4) \text {, such that } \\
\Psi\left(P_{Q, g}\right) \text { is the mean reward of the optimal TR under } Q\end{array}$ \\
\hline$\Psi_{r_{0}}$ & $\begin{array}{l}\text { mapping from } \mathcal{M} \text { to }[0,1] \text { given by }(4.16) \text {, such that } \\
\Psi_{r_{0}}\left(P_{Q, g}\right) \text { is the mean reward of TR } r_{0} \text { under } Q \text { (note } \\
\text { that } r_{0} \text { may differ from } r\left(Q_{Y}\right) \text { ) }\end{array}$ \\
\hline$Q, Q_{0}$ & $\begin{array}{l}\text { generic and true infinite-dimensional parameters fully de- } \\
\text { scribing the marginal distribution of } W \text { and conditional } \\
\text { distribution of } Y \text { given } A, W\end{array}$ \\
\hline$Q_{0}^{\star}$ & $\begin{array}{l}\text { infinite-dimensional parameter such that } W \text { has the same } \\
\text { marginal distribution under } Q_{0}^{\star} \text { as under } Q_{0} \text { and the con- } \\
\text { ditional distribution of } Y \text { given } A, W \text { under } Q_{0} \text { is } Q_{Y, 0}^{\star}\end{array}$ \\
\hline$Q_{Y}, Q_{Y, 0}$ & $\begin{array}{l}\text { (generic and true) conditional expectations of } Y \text { given } \\
A, W \text { under } Q \text { and } Q_{0}\end{array}$ \\
\hline$Q_{Y, 0}^{\star}$ & $\begin{array}{l}\text { shorthand notation for } Q_{Y, \beta_{0}, g_{0}, r_{0}}\left(\epsilon_{0}\left(r_{0}\right)\right) \text {, a conditional } \\
\text { expectation of } Y \text { given } A, W \text { defined as the optimal fluc- } \\
\text { tuation of } Q_{Y, \beta_{0}, g_{0}} \text { in the direction of } H_{r_{0}}\left(g_{0}\right) \text {, see }(4.8) \text {; } \\
\text { such that } E_{Q_{0}}\left(Q_{Y, 0}^{\star}\left(r_{0}(W), W\right)\right) \text { equals } \psi_{0}\end{array}$ \\
\hline$Q_{Y, \beta}$ & generic element of $\mathcal{Q}_{1}$ \\
\hline$Q_{Y, \beta_{n, 0}}, Q_{Y, \beta_{0}}$ & projections of $Q_{Y, 0}$ onto $\mathcal{Q}_{1, n}$ and $\mathcal{Q}_{1}$, see $\mathbf{A} 2$ \\
\hline
\end{tabular}


$Q_{Y, \beta_{i}}, Q_{Y, \beta_{n}} \quad$ estimators of $Q_{Y, 0}$ defined as solutions to the minimization of the weighted empirical risk over $\mathcal{Q}_{1, i}$ and $\mathcal{Q}_{1, n}$ at sample sizes $i$ and $n$, see (2.8)

$Q_{Y, \beta, g, \rho}(\epsilon) \quad$ generic fluctuation, indexed by $\epsilon$, of $Q_{Y, \beta}$ in the direction of $H_{\rho}(g)$, see $(2.15)$ :

$$
\begin{aligned}
Q_{Y, \beta, g, \rho}(\epsilon) & \equiv \operatorname{expit}\left(\operatorname{logit}\left(Q_{Y, \beta}\right)+\epsilon H_{\rho}(g)\right) \\
& =\operatorname{expit}\left(\operatorname{logit}\left(Q_{Y, \beta}\right)+\epsilon \frac{\mathbf{1}\{A=\rho(W)\}}{g(A \mid W)}\right)
\end{aligned}
$$

$Q_{Y, \beta_{0}, g_{0}, \rho}(\epsilon) \quad$ generic fluctuation, indexed by $\epsilon$, of $Q_{Y, \beta_{0}}$ in the direction of $H_{\rho}\left(g_{0}\right)$, see (4.2)

$Q_{Y, \beta_{n}, g_{n}, r_{n}}(\epsilon) \quad$ fluctuation, indexed by $\epsilon$, of the initial estimator $Q_{Y, \beta_{n}}$ of $Q_{Y, 0}$ in the direction of $H_{r_{n}}\left(g_{n}\right)$, see (2.15)

$Q_{Y, \beta_{n}, g_{n}, r_{n}}^{\star}$

targeted, empirically optimal fluctuation $Q_{Y, \beta_{n}, g_{n}, r_{n}}\left(\epsilon_{n}\right)$ of the initial estimator $Q_{Y, \beta_{n}}$ of $Q_{Y, 0}$ in the direction of $H_{r_{n}}\left(g_{n}\right)$

$Q_{Y, \zeta_{n}, r_{n}}^{\star}$

$Q_{Y, \zeta, \rho}(\epsilon)$ shorthand notation for $Q_{Y, \beta_{n}, g_{n}, r_{n}}^{\star}$ (used in the supplementary article only)

shorthand notation for $Q_{Y, \beta, g, \rho}(\epsilon)$, see (A.1) (used in the supplementary article only)

$Q_{Y, \zeta, \rho}(\epsilon) \circ \rho \quad$ shorthand notation defined in (A.2) (used in the supplementary article only)

$q_{Y}, q_{Y, 0}$ (generic and true) blip functions under $Q$ and $Q_{0}$, given by $q_{Y}(W) \equiv Q_{Y}(1, W)-Q_{Y}(0, W)$ and $q_{Y, 0}(W) \equiv$ $Q_{Y, 0}(1, W)-Q_{Y, 0}(0, W)$

$q_{Y, \beta}$ blip function associated with $Q_{Y, \beta}$, given by $q_{Y, \beta}(W) \equiv$ $Q_{Y, \beta}(1, W)-Q_{Y, \beta}(0, W)$

$q_{Y, \beta_{i}}, q_{Y, \beta_{n}} \quad$ estimators of $q_{Y, 0}$ defined as the blip functions associated with $Q_{Y, \beta_{i}}, Q_{Y, \beta_{n}}$, see (2.9)

$\mathcal{Q}, \mathcal{Q}_{Y}$ semiparametric collections of all $Q_{\mathrm{s}}$ and all $Q_{Y} \mathrm{~s}$

$\mathcal{Q}_{1, n}, \mathcal{Q}_{1}$ $n$th working model $\mathcal{Q}_{1, n} \equiv\left\{Q_{Y, \beta}: \beta \in B_{n}\right\} \subset \mathcal{Q}_{Y}$ and their union $\mathcal{Q}_{1} \equiv \cup_{n \geq 1} \mathcal{Q}_{1, n}$

$r\left(Q_{Y}\right)$ optimal TR under $Q$, see (2.2)

$r\left(Q_{Y, 0}\right)$ or $r_{0}$ optimal TR under $Q_{0}$, given by $r\left(Q_{Y, 0}\right)(W) \equiv r_{0}(W) \equiv$ $\mathbf{1}\left\{q_{Y, 0}(W) \geq 0\right\}$

$r_{i}, r_{n}$ estimators of $r_{0}$ defined as the TRs associated with $q_{Y, \beta_{i}}$ and $q_{Y, \beta_{n}}$ at sample sizes $i$ and $n$, see (2.10) 


\section{$\rho$ \\ $\mathcal{R}$ \\ $r\left(\mathcal{Q}_{1, n}\right)$ \\ $\Sigma_{0}, \Sigma_{n}$ \\ $\Sigma_{0}^{\mathcal{E}}, \Sigma_{n}^{\mathcal{E}}$}

stochastic TR

$t_{n}$

treatment rule

generic TR $\rho \in \mathcal{R}$

set of all TRs

the subset of $\mathcal{R}$ defined as

$r\left(\mathcal{Q}_{1, n}\right) \equiv\left\{r\left(Q_{Y}\right): Q_{Y} \in \mathcal{Q}_{1, n}\right\}$

asymptotic variance of $\sqrt{n}\left(\psi_{n}^{\star}-\psi_{r_{n}, 0}\right)$ and an explicit estimator used to derive confidence intervals for $\psi_{r_{n}, 0}$ and $\psi_{0}$, see (4.11) and (4.14)

asymptotic variance of $\sqrt{n}\left(\psi_{n}^{\star}+\mathcal{E}_{n}-n^{-1} \sum_{i=1}^{n} Y_{i}\right)$ and an explicit estimator used to derive lower confidence bounds for $\mathcal{E}_{n}$ and $\mathcal{C}_{n}$, see (5.3) and (5.4)

individualized treatment strategy in which treatment assignment for a patient is determined randomly from a conditional distribution given her measured baseline covariates; generically denoted $g$

fine-tune parameter used in the definition of $G_{n}$

individualized treatment strategy in which treatment assignment for a patient is determined deterministically based on her measured baseline covariates; generically denoted $\rho$, a function of baseline covariates prescribing deterministically which treatment to assign; can be seen as a degenerate stochastic TR

TR abbreviation of "treatment rule"

$\xi_{n}$ $\zeta, \zeta_{0}, \zeta_{n}$ fine-tune parameter used in the definition of $G_{n}$ shorthand notation used for a generic indexing parameter $\zeta \equiv(\beta, g), \zeta_{0} \equiv\left(\beta_{0}, g_{0}\right)$ and $\zeta_{n} \equiv\left(\beta_{n}, g_{n}\right)$ (used in the supplemental article only)

$Z$ given a known $g \in \mathcal{G}$ and $O$ drawn from $P_{Q_{0}, g}, Z \equiv$ $g(A \mid W)$ is a weight associated with $O$; since $Z$ is deterministically determined given $g$ and $O$, we can augment $O$ with $Z$, i.e., substitute $(O, Z)$ for $O$ and still say that $(O, Z)$ is drawn from $P_{Q_{0}, g}$

$Z_{i}, Z_{n} \quad Z_{i} \equiv g_{i}\left(A_{i} \mid W_{i}\right)$ and $Z_{n} \equiv g_{n}\left(A_{n} \mid W_{n}\right)$ are weights associated with $O_{i}$ and $O_{n}$; we can substitute $\left(O_{i}, Z_{i}\right)$ and $\left(O_{n}, Z_{n}\right)$ for $O_{i}$ and $O_{n}$ and still say that $\left(O_{i}, Z_{i}\right)$ and $\left(O_{n}, Z_{n}\right)$ are drawn from $P_{Q_{0}, g_{i}}$ and $P_{Q_{0}, g_{i}}$ conditionally on $\mathbf{O}(i-1)$ and $\mathbf{O}(n-1)$ 


\section{REFERENCES}

[1] E. Bolthausen, E. Perkins, and A. van der Vaart. Lectures on probability theory and statistics, volume 1781 of Lecture Notes in Mathematics. Springer-Verlag, Berlin, 2002. Lectures from the 29th Summer School on Probability Theory held in Saint-Flour, July 8-24, 1999, Edited by P. Bernard.

[2] V. H. de la Peña and E. Giné. Decoupling. Probability and its Applications (New York). Springer-Verlag, New York, 1999.

[3] A. R. Luedtke and M. J. van der Laan. Targeted learning of the mean outcome under an optimal dynamic treatment rule. Journal of Causal Inference, 3(1):61-95, 2015.

[4] A. R. Luedtke and M. J. van der Laan. Statistical inference for the mean outcome under a possibly non-unique optimal treatment strategy. Ann. Statist., 44(2):713-742, 2016.

[5] P. K. Sen and J. M. Singer. Large sample methods in statistics. Chapman \& Hall, New York, 1993. An introduction with applications.

[6] A. W. van der Vaart. Asymptotic statistics, volume 3 of Cambridge Series in Statistical and Probabilistic Mathematics. Cambridge University Press, Cambridge, 1998.

[7] A. W. van der Vaart and J. A. Wellner. Weak convergence and empirical processes. Springer Series in Statistics. Springer-Verlag, New York, 1996. With applications to statistics.

[8] W. Zheng, A. Chambaz, and M. J. van der Laan. Drawing valid targeted inference when covariate-adjusted response-adaptive RCT meets data-adaptive loss-based estimation, with an application to the LASSO. Technical Report 339, U.C. Berkeley Division of Biostatistics Working Paper Series, 2015.

MODAL'X

UPL, Université Paris Nanterre

F92000 NANTERRE

France

E-MAIL: achambaz@u-paris10.fr
Division of Biostatistics

AND

Center for Targeted Machine

Learning and Causal Inference

University of CAlifornia, Berkeley

101 Haviland Hall

Berkeley, California 94720

USA

E-MAIL: wenjing.zheng@berkeley.edu laan@berkeley.edu 